\title{
Catalyst-Controlled Selective Alkylation/Cyclopropanation of Indoles with Vinyl Diazoesters
}

\author{
Zhen Wang, Guangyang Xu, Shengbiao Tang, Ying Shao and Jiangtao Sun* \\ Jiangsu Key Laboratory of Advanced Catalytic Materials \& Technology, School of \\ Petrochemical Engineering, Changzhou University, Changzhou 213164, China \\ E-mail: jtsun@cczu.edu.cn or jtsun08@gmail.com
}

Table of Contents

1. General information

2. General procedure for Scheme 2

3. General procedure for Scheme 3

4. Further exploration for Scheme 4

5. X-ray structure of $\mathbf{3 a}$ and $\mathbf{4 a}$

6. References

7. ${ }^{1} \mathrm{H}$ NMR, ${ }^{13} \mathrm{C}$ NMR and ${ }^{19} \mathrm{~F}$ NMR Spectra of compounds 


\section{General information}

All of the reactions were carried out in flame-dried tubes under argon atmosphere. Solvents were dried prior to use. For column chromatography, 200-300 mesh silica gel was used. ${ }^{1} \mathrm{H}$ NMR were recorded on Bruker $300 \mathrm{MHz}$ or $400 \mathrm{MHz}$ spectrometer in $\mathrm{CDCl}_{3}$ or DMSO- $\mathrm{d}_{6} .{ }^{13} \mathrm{C}$ NMR were recorded on Bruker $75 \mathrm{MHz}$ or $100 \mathrm{MHz}$ spectrometer in $\mathrm{CDCl}_{3} .{ }^{19} \mathrm{~F}$ NMR were recorded on Bruker $282 \mathrm{MHz}$ spectrometer in $\mathrm{CDCl}_{3}$. HRMS were performed on Agilent 6540 Q-TOF mass spectrometer (ESI). Melting points were determined on a SGW X-4B melting point apparatus. Indole compounds $(\mathbf{1})^{1}$ and diazo compounds $(\mathbf{2})^{2}$ were known compounds and prepared according to the literature procedures.

\section{General procedure for Scheme 2}

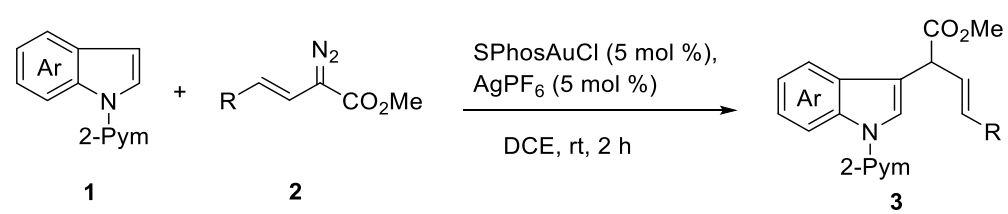

To a dry Schlenk tube was added $1(0.1 \mathrm{mmol}), \mathrm{SPhosAuCl}(0.005 \mathrm{mmol}), \mathrm{AgPF}_{6}(0.005 \mathrm{mmol})$ and DCE $(1 \mathrm{~mL})$. The mixture was stirred at $\mathrm{rt}$ for $0.5 \mathrm{~h}$ under an argon atmosphere, then $2(0.2$ mmol) in $1 \mathrm{~mL}$ of DCE was added via a syringe pump over $2 \mathrm{~h}$. The reaction mixture was concentrated under vacuum, the residue was purified by column chromatography (silica gel, petroleum ether/ethyl acetate $=20: 1$ to 10:1) to give 3 .

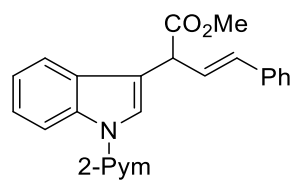

\section{Methyl (E)-4-phenyl-2-(1-(pyrimidin-2-yl)-1H-indol-3-yl)but-3-enoate (3a):}

Obtained as a white solid after column chromatography (petroleum ether/ethyl acetate $=20: 1$ to 10:1), $31 \mathrm{mg}, 85 \%$ yield, mp: $136-137{ }^{\circ} \mathrm{C} .{ }^{1} \mathrm{H}$ NMR $\left(400 \mathrm{MHz}, \mathrm{CDCl}_{3}\right) \delta 8.82(\mathrm{~d}, J=8.3 \mathrm{~Hz}, 1 \mathrm{H})$, $8.69(\mathrm{~d}, J=4.8 \mathrm{~Hz}, 2 \mathrm{H}), 8.30(\mathrm{~s}, 1 \mathrm{H}), 7.66(\mathrm{~d}, J=7.8 \mathrm{~Hz}, 1 \mathrm{H}), 7.27-7.41(\mathrm{~m}, 5 \mathrm{H}), 7.25-7.21(\mathrm{~m}$, 2H), $7.04(\mathrm{t}, J=4.7 \mathrm{~Hz}, 1 \mathrm{H}), 6.67(\mathrm{dd}, J=15.8,7.6 \mathrm{~Hz}, 1 \mathrm{H}), 6.62(\mathrm{~d}, J=15.8 \mathrm{~Hz}, 1 \mathrm{H}), 4.79(\mathrm{~d}, J$ $=7.6 \mathrm{~Hz}, 1 \mathrm{H}), 3.76(\mathrm{~s}, 3 \mathrm{H}) .{ }^{13} \mathrm{C} \mathrm{NMR}\left(100 \mathrm{MHz}, \mathrm{CDCl}_{3}\right) \delta 172.8,158.2,157.7,136.8,135.9$, 132.9, 129.9, 128.7, 127.8, 126.7, 126.0, 124.2, 124.1, 122.3, 119.4, 117.2, 116.6, 116.3, 52.6, 46.8. HRMS (ESI) calcd. for $\mathrm{C}_{23} \mathrm{H}_{20} \mathrm{~N}_{3} \mathrm{O}_{2}[\mathrm{M}+\mathrm{H}]^{+}: 370.1550$, found: 370.1554 .

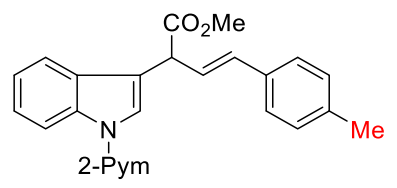

Methyl (E)-2-(1-(pyrimidin-2-yl)-1H-indol-3-yl)-4-(p-tolyl)but-3-enoate (3b):

Obtained as a white solid after column chromatography (petroleum ether/ethyl acetate $=20: 1$ to 10:1), $32 \mathrm{mg}, 83 \%$ yield, mp: $126-127^{\circ} \mathrm{C} .{ }^{1} \mathrm{H}$ NMR $\left(300 \mathrm{MHz}, \mathrm{CDCl}_{3}\right) \delta 8.81(\mathrm{~d}, J=8.3 \mathrm{~Hz}, 1 \mathrm{H})$, $8.67(\mathrm{~d}, J=4.8 \mathrm{~Hz}, 2 \mathrm{H}), 8.29(\mathrm{~d}, J=0.5 \mathrm{~Hz}, 1 \mathrm{H}), 7.66(\mathrm{~d}, J=7.5 \mathrm{~Hz}, 1 \mathrm{H}), 7.38-7.22(\mathrm{~m}, 4 \mathrm{H})$, $7.11(\mathrm{~d}, J=7.9 \mathrm{~Hz}, 2 \mathrm{H}), 7.01(\mathrm{t}, J=4.8 \mathrm{~Hz}, 1 \mathrm{H}), 6.68-6.56(\mathrm{~m}, 2 \mathrm{H}), 4.77(\mathrm{~d}, J=6.1 \mathrm{~Hz}, 1 \mathrm{H}), 3.75$ 
(s, 3H), $2.32(\mathrm{~s}, 3 \mathrm{H}) .{ }^{13} \mathrm{C}$ NMR $\left(75 \mathrm{MHz}, \mathrm{CDCl}_{3}\right) \delta 172.9,158.2,157.7,137.7,135.9,134.0,132.8$, 130.0, 129.4, 126.6, 124.9, 124.2, 124.1, 122.3, 119.4, 117.3, 116.6, 116.3, 52.5, 46.8, 21.3. HRMS (ESI) calcd. for $\mathrm{C}_{24} \mathrm{H}_{22} \mathrm{~N}_{3} \mathrm{O}_{2}[\mathrm{M}+\mathrm{H}]^{+}: 384.1707$, found: 384.1703 .

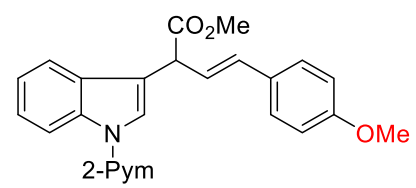

Methyl (E)-4-(4-methoxyphenyl)-2-(1-(pyrimidin-2-yl)-1H-indol-3-yl)but-3-enoate (3c):

Obtained as a white solid after column chromatography (petroleum ether/ethyl acetate $=20: 1$ to 10:1), $31 \mathrm{mg}, 77 \%$ yield, mp: $123-124{ }^{\circ} \mathrm{C} .{ }^{1} \mathrm{H}$ NMR $\left(400 \mathrm{MHz}, \mathrm{CDCl}_{3}\right) \delta 8.81(\mathrm{~d}, J=8.3 \mathrm{~Hz}, 1 \mathrm{H})$, $8.67(\mathrm{~d}, J=4.8 \mathrm{~Hz}, 2 \mathrm{H}), 8.29(\mathrm{~s}, 1 \mathrm{H}), 7.66(\mathrm{~d}, J=7.8 \mathrm{~Hz}, 1 \mathrm{H}), 7.37-7.32(\mathrm{~m}, 3 \mathrm{H}), 7.27-7.23$ (t, $1 \mathrm{H}), 7.02(\mathrm{t}, J=4.8 \mathrm{~Hz}, 1 \mathrm{H}), 6.83(\mathrm{~d}, J=8.6 \mathrm{~Hz}, 2 \mathrm{H}), 6.60-6.51(\mathrm{~m}, 2 \mathrm{H}), 4.76(\mathrm{~d}, J=6.1 \mathrm{~Hz}, 1 \mathrm{H})$, $3.79(\mathrm{~s}, 3 \mathrm{H}), 3.75(\mathrm{~s}, 3 \mathrm{H}) .{ }^{13} \mathrm{C} \mathrm{NMR}\left(75 \mathrm{MHz}, \mathrm{CDCl}_{3}\right) \delta 173.0,159.4,158.2,157.8,135.9,132.3$, 123.0, 129.6, 127.9, 124.2, 124.0, 123.8, 122.3, 119.4, 117.4, 116.6, 116.2, 114.1, 55.4, 52.5, 46.8 . HRMS (ESI) calcd. for $\mathrm{C}_{24} \mathrm{H}_{22} \mathrm{~N}_{3} \mathrm{O}_{3}[\mathrm{M}+\mathrm{H}]^{+}: 400.1656$, found: 400.1659 .

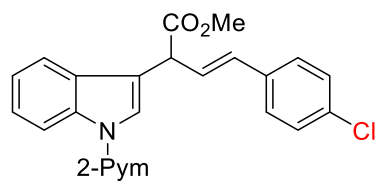

\section{Methyl (E)-4-(4-chlorophenyl)-2-(1-(pyrimidin-2-yl)-1H-indol-3-yl)but-3-enoate (3d):}

Obtained as a white solid after column chromatography (petroleum ether/ethyl acetate $=20: 1$ to 10:1), $32 \mathrm{mg}, 78 \%$ yield, mp: $150-151{ }^{\circ} \mathrm{C} .{ }^{1} \mathrm{H}$ NMR $\left(300 \mathrm{MHz}, \mathrm{CDCl}_{3}\right) \delta 8.82(\mathrm{~d}, J=8.3 \mathrm{~Hz}, 1 \mathrm{H})$, $8.66(\mathrm{~d}, J=4.8 \mathrm{~Hz}, 2 \mathrm{H}), 8.29(\mathrm{~s}, 1 \mathrm{H}), 7.64(\mathrm{~d}, J=7.4 \mathrm{~Hz}, 1 \mathrm{H}), 7.39-7.30(\mathrm{~m}, 2 \mathrm{H}), 7.29-7.22(\mathrm{~m}$, 4H), $7.01(\mathrm{t}, J=4.8 \mathrm{~Hz}, 1 \mathrm{H}), 6.67(\mathrm{dd}, J=15.8,7.6 \mathrm{~Hz}, 1 \mathrm{H}), 6.55(\mathrm{~d}, J=15.8 \mathrm{~Hz}, 1 \mathrm{H}), 4.78(\mathrm{~d}, J$ $=7.6 \mathrm{~Hz}, 1 \mathrm{H}), 3.75(\mathrm{~s}, 3 \mathrm{H}) .{ }^{13} \mathrm{C} \mathrm{NMR}\left(75 \mathrm{MHz}, \mathrm{CDCl}_{3}\right) \delta 172.7,158.2,157.7,135.9,135.3,133.4$, 131.6, 129.8, 128.8, 127.9, 126.7, 124.3, 124.1, 122.4, 119.3, 116.9, 116.6, 116.3, 52.6, 46.7. HRMS (ESI) calcd. for $\mathrm{C}_{23} \mathrm{H}_{19} \mathrm{ClN}_{3} \mathrm{O}_{2}[\mathrm{M}+\mathrm{H}]^{+}:$404.1160, found: 404.1165 .

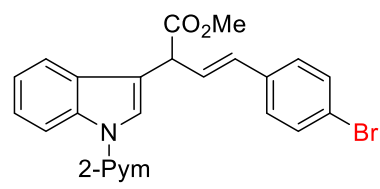

Methyl (E)-4-(4-bromophenyl)-2-(1-(pyrimidin-2-yl)-1H-indol-3-yl)but-3-enoate (3e):

Obtained as a white solid after column chromatography (petroleum ether/ethyl acetate $=20: 1$ to 10:1), $27 \mathrm{mg}, 59 \%$ yield, mp: $153-154{ }^{\circ} \mathrm{C} .{ }^{1} \mathrm{H}$ NMR $\left(400 \mathrm{MHz}, \mathrm{CDCl}_{3}\right) \delta 8.81(\mathrm{~d}, J=8.4 \mathrm{~Hz}, 1 \mathrm{H})$, $8.67(\mathrm{~d}, J=4.8 \mathrm{~Hz}, 2 \mathrm{H}), 8.29(\mathrm{~s}, 1 \mathrm{H}), 7.64(\mathrm{~d}, J=7.8 \mathrm{~Hz}, 1 \mathrm{H}), 7.41-7.34(\mathrm{~m}, 3 \mathrm{H}), 7.27-7.23(\mathrm{~m}$, $3 \mathrm{H}), 7.01(\mathrm{t}, J=4.8 \mathrm{~Hz}, 1 \mathrm{H}), 6.69(\mathrm{dd}, J=15.8,7.9 \mathrm{~Hz}, 1 \mathrm{H}), 6.53(\mathrm{~d}, J=15.8 \mathrm{~Hz}, 1 \mathrm{H}), 4.77(\mathrm{~d}, J$ $=7.9 \mathrm{~Hz}, 1 \mathrm{H}), 3.76(\mathrm{~s}, 3 \mathrm{H}) \cdot{ }^{13} \mathrm{C} \mathrm{NMR}\left(75 \mathrm{MHz}, \mathrm{CDCl}_{3}\right) \delta 172.6,158.2,157.7,135.9,135.7,131.7$, 131.6, 129.8, 128.2, 126.9, 124.3, 124.1, 122.4, 121.6, 119.3, 116.8, 116.6, 116.3, 52.6, 46.7. HRMS (ESI) calcd. for $\mathrm{C}_{23} \mathrm{H}_{19} \mathrm{BrN}_{3} \mathrm{O}_{2}[\mathrm{M}+\mathrm{H}]^{+}: 448.0655$, found: 448.0659 . 


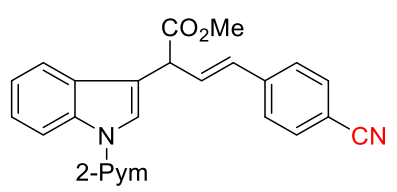

Methyl (E)-4-(4-cyanophenyl)-2-(1-(pyrimidin-2-yl)-1H-indol-3-yl)but-3-enoate (3f):

Obtained as a white solid after column chromatography (petroleum ether/ethyl acetate $=10: 1$ to 5:1), $28 \mathrm{mg}, 71 \%$ yield, mp: $156-157^{\circ} \mathrm{C} .{ }^{1} \mathrm{H}$ NMR $\left(400 \mathrm{MHz}, \mathrm{CDCl}_{3}\right) \delta 8.82(\mathrm{~d}, J=8.4 \mathrm{~Hz}, 1 \mathrm{H})$, $8.68(\mathrm{~d}, J=4.8 \mathrm{~Hz}, 2 \mathrm{H}), 8.30(\mathrm{~s}, 1 \mathrm{H}), 7.63(\mathrm{~d}, J=7.8 \mathrm{~Hz}, 1 \mathrm{H}), 7.55(\mathrm{~d}, J=8.3 \mathrm{~Hz}, 2 \mathrm{H}), 7.43(\mathrm{~d}, J$ $=8.3 \mathrm{~Hz}, 2 \mathrm{H}), 7.39-7.35(\mathrm{~m}, 1 \mathrm{H}), 7.28-7.24(\mathrm{~m}, 1 \mathrm{H}), 7.03(\mathrm{t}, J=4.8 \mathrm{~Hz}, 1 \mathrm{H}), 6.83(\mathrm{dd}, J=15.9$, $7.8 \mathrm{~Hz}, 1 \mathrm{H}), 6.58(\mathrm{~d}, J=15.9 \mathrm{~Hz}, 1 \mathrm{H}), 4.81(\mathrm{~d}, J=7.8 \mathrm{~Hz}, 1 \mathrm{H}), 3.77(\mathrm{~s}, 3 \mathrm{H}) .{ }^{13} \mathrm{C}$ NMR $(75 \mathrm{MHz}$, $\left.\mathrm{CDCl}_{3}\right) \delta 172.3,158.2,157.6,141.2,135.8,132.4,131.1,130.2,129.6,127.1,124.3,124.2,122.4$, 119.1, 119.0, 116.7, 116.4, 116.3, 111.0, 52.7, 46.5. HRMS (ESI) calcd. for $\mathrm{C}_{24} \mathrm{H}_{19} \mathrm{~N}_{4} \mathrm{O}_{2}[\mathrm{M}+\mathrm{H}]^{+}$: 395.1503, found: 395.1507 .

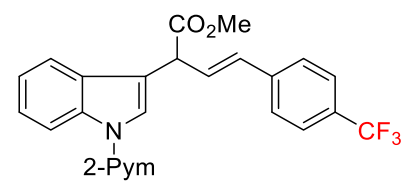

Methyl (E)-2-(1-(pyrimidin-2-yl)-1H-indol-3-yl)-4-(4-(trifluoromethyl)phenyl)but-3-enoate (3g): Obtained as a white solid after column chromatography (petroleum ether/ethyl acetate $=20: 1$ to 10:1), $30 \mathrm{mg}, 69 \%$ yield, mp: $176-177{ }^{\circ} \mathrm{C} .{ }^{1} \mathrm{H}$ NMR $\left(300 \mathrm{MHz}, \mathrm{CDCl}_{3}\right) \delta 8.83(\mathrm{~d}, J=8.3 \mathrm{~Hz}, 1 \mathrm{H})$, $8.68(\mathrm{~d}, J=4.8 \mathrm{~Hz}, 2 \mathrm{H}), 8.31(\mathrm{~s}, 1 \mathrm{H}), 7.65(\mathrm{~d}, J=7.5 \mathrm{~Hz}, 1 \mathrm{H}), 7.54$ (d, $J=8.3 \mathrm{~Hz}, 2 \mathrm{H}), 7.47$ (d, $J$ $=8.3 \mathrm{~Hz}, 2 \mathrm{H}), 7.40-7.34(\mathrm{~m}, 1 \mathrm{H}), 7.29-7.23(\mathrm{~m}, 1 \mathrm{H}), 7.03(\mathrm{t}, J=4.8 \mathrm{~Hz}, 1 \mathrm{H}), 6.80(\mathrm{dd}, J=15.9$, $7.7 \mathrm{~Hz}, 1 \mathrm{H}), 6.63(\mathrm{~d}, J=15.9 \mathrm{~Hz}, 1 \mathrm{H}), 4.82(\mathrm{~d}, J=7.7 \mathrm{~Hz}, 1 \mathrm{H}), 3.77(\mathrm{~s}, 3 \mathrm{H}) .{ }^{13} \mathrm{C} \mathrm{NMR}(75 \mathrm{MHz}$, $\left.\mathrm{CDCl}_{3}\right) \delta 172.5,158.2,157.7,140.3,135.9,131.5,129.8,129.8,129.4,128.8,126.8,126.1,125.7$, 125.6, 125.6, 125.5, 124.3, 124.2, 122.5, 122.4, 119.3, 116.7, 116.6, 116.4, 52.7, 46.6. ${ }^{19} \mathrm{~F}$ NMR $\left(282 \mathrm{MHz}, \mathrm{CDCl}_{3}\right) \delta$-62.5. HRMS (ESI) calcd. for $\mathrm{C}_{24} \mathrm{H}_{19} \mathrm{~F}_{3} \mathrm{~N}_{3} \mathrm{O}_{2}[\mathrm{M}+\mathrm{H}]^{+}$: 438.1424, found: 438.1426

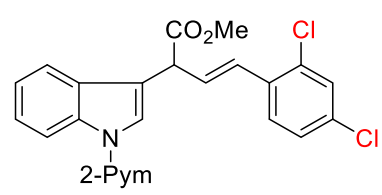

Methyl (E)-4-(2,4-dichlorophenyl)-2-(1-(pyrimidin-2-yl)-1H-indol-3-yl)but-3-enoate (3h):

Obtained as a white solid after column chromatography (petroleum ether/ethyl acetate $=20: 1$ to 10:1), $26 \mathrm{mg}, 59 \%$ yield, mp: $153-154{ }^{\circ} \mathrm{C} .{ }^{1} \mathrm{H}$ NMR $\left(300 \mathrm{MHz}, \mathrm{CDCl}_{3}\right) \delta 8.82(\mathrm{~d}, J=8.3 \mathrm{~Hz}, 1 \mathrm{H})$, $8.68(\mathrm{~d}, J=4.8 \mathrm{~Hz}, 2 \mathrm{H}), 8.30(\mathrm{~s}, 1 \mathrm{H}), 7.68(\mathrm{~d}, J=7.4 \mathrm{~Hz}, 1 \mathrm{H}), 7.51(\mathrm{~d}, J=8.5 \mathrm{~Hz}, 1 \mathrm{H}), 7.39-7.34$ (m, 2H), 7.29-7.24 (m, 1H), $7.18(\mathrm{dd}, J=8.2,1.9 \mathrm{~Hz}, 1 \mathrm{H}), 7.04(\mathrm{t}, J=4.8 \mathrm{~Hz}, 1 \mathrm{H}), 6.96(\mathrm{~d}, J=$ $15.8 \mathrm{~Hz}, 1 \mathrm{H}), 6.66(\mathrm{dd}, J=15.8,8.3 \mathrm{~Hz}, 1 \mathrm{H}), 4.83(\mathrm{~d}, J=8.3 \mathrm{~Hz}, 1 \mathrm{H}), 3.77(\mathrm{~s}, 3 \mathrm{H}) .{ }^{13} \mathrm{C}$ NMR $(75$ $\left.\mathrm{MHz}, \mathrm{CDCl}_{3}\right) \delta 172.5,158.3,157.7,135.9,133.8,133.7,133.6,129.7,129.5,128.1,128.0,127.3$, 124.3, 124.0, 122.4, 119.3, 116.8, 116.7, 116.4, 52.7, 46.9. HRMS (ESI) calcd. for $\mathrm{C}_{23} \mathrm{H}_{18} \mathrm{Cl}_{2} \mathrm{~N}_{3} \mathrm{O}_{2}$ $[\mathrm{M}+\mathrm{H}]^{+}:$438.0771, found: 438.0774 . 


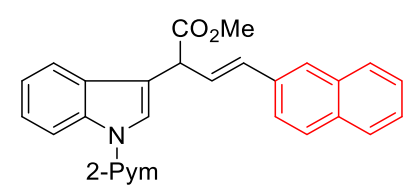

Methyl (E)-4-(naphthalen-2-yl)-2-(1-(pyrimidin-2-yl)-1H-indol-3-yl)but-3-enoate (3i):

Obtained as a white solid after column chromatography (petroleum ether/ethyl acetate $=20: 1$ to 10:1), $31 \mathrm{mg}, 73 \%$ yield, mp: $100-101{ }^{\circ} \mathrm{C} .{ }^{1} \mathrm{H}$ NMR $\left(300 \mathrm{MHz}, \mathrm{CDCl}_{3}\right) \delta 8.82(\mathrm{~d}, J=8.3 \mathrm{~Hz}, 1 \mathrm{H})$, $8.65(\mathrm{~d}, J=4.8 \mathrm{~Hz}, 2 \mathrm{H}), 8.34(\mathrm{~s}, 1 \mathrm{H}), 7.77-7.68(\mathrm{~m}, 5 \mathrm{H}), 7.64-7.60(\mathrm{~m}, 1 \mathrm{H}), 7.45-7.34(\mathrm{~m}, 3 \mathrm{H})$, 7.29-7.23 (m, 1H), $6.98(\mathrm{t}, J=4.8 \mathrm{~Hz}, 1 \mathrm{H}), 6.87-6.74(\mathrm{~m}, 2 \mathrm{H}), 4.85(\mathrm{~d}, J=6.2 \mathrm{~Hz}, 1 \mathrm{H}), 3.77(\mathrm{~s}$, $3 \mathrm{H}) .{ }^{13} \mathrm{C}$ NMR $\left(75 \mathrm{MHz}, \mathrm{CDCl}_{3}\right) \delta 172.9,158.2,157.7,135.9,134.3,133.6,133.1,133.0,129.9$, $128.3,128.1,127.8,126.6,126.4,126.4,126.0,124.2,124.1,123.8,122.4,119.4,117.2,116.6$, 116.3, 52.6, 46.9. HRMS (ESI) calcd. for $\mathrm{C}_{27} \mathrm{H}_{22} \mathrm{~N}_{3} \mathrm{O}_{2}[\mathrm{M}+\mathrm{H}]^{+}:$420.1707, found: 420.1703 .

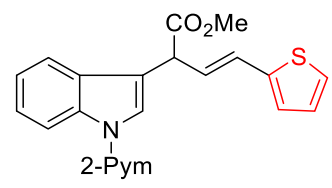

Methyl (E)-2-(1-(pyrimidin-2-yl)-1H-indol-3-yl)-4-(thiophen-2-yl)but-3-enoate (3j):

Obtained as a light yellow solid after column chromatography (petroleum ether/ethyl acetate $=$ 20:1 to 10:1), $24 \mathrm{mg}, 63 \%$ yield, mp: $137-138{ }^{\circ} \mathrm{C} .{ }^{1} \mathrm{H}$ NMR $\left(300 \mathrm{MHz}, \mathrm{CDCl}_{3}\right) \delta 8.81(\mathrm{~d}, J=8.3$ $\mathrm{Hz}, 1 \mathrm{H}), 8.66(\mathrm{~d}, J=4.8 \mathrm{~Hz}, 2 \mathrm{H}), 8.30(\mathrm{~s}, 1 \mathrm{H}), 7.64(\mathrm{~d}, J=7.5 \mathrm{~Hz}, 1 \mathrm{H}), 7.38-7.33(\mathrm{~m}, 1 \mathrm{H})$, 7.28-7.22 (m, 1H), 7.15-7.12 (m, 1H), $7.00(\mathrm{t}, J=4.8 \mathrm{~Hz}, 1 \mathrm{H}), 6.92(\mathrm{~m}, 2 \mathrm{H}), 6.73(\mathrm{~d}, J=15.7 \mathrm{~Hz}$, $1 \mathrm{H}), 6.52(\mathrm{dd}, J=15.7,7.7 \mathrm{~Hz}, 1 \mathrm{H}), 4.75(\mathrm{~d}, J=7.7 \mathrm{~Hz}, 1 \mathrm{H}), 3.75(\mathrm{~s}, 3 \mathrm{H}) .{ }^{13} \mathrm{C}$ NMR $(75 \mathrm{MHz}$, $\left.\mathrm{CDCl}_{3}\right) \delta 172.6,158.2,157.7,141.8,135.9,129.9,127.4,126.1,126.0,125.5,124.6,124.2,122.4$, 119.3, 116.8, 116.6, 116.3, 52.6, 46.5. HRMS (ESI) calcd. for $\mathrm{C}_{21} \mathrm{H}_{18} \mathrm{~N}_{3} \mathrm{O}_{2} \mathrm{~S}[\mathrm{M}+\mathrm{H}]^{+}$: 376.1114 , found: 376.1111 .

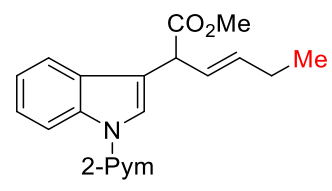

Methyl (E)-2-(1-(pyrimidin-2-yl)-1H-indol-3-yl)hex-3-enoate (3k):

Obtained as colorless oil after column chromatography (petroleum ether/ethyl acetate $=20: 1$ to 10:1), $10 \mathrm{mg}, 31 \%$ yield. ${ }^{1} \mathrm{H}$ NMR $\left(400 \mathrm{MHz}, \mathrm{CDCl}_{3}\right) \delta 8.80(\mathrm{~d}, J=8.4 \mathrm{~Hz}, 1 \mathrm{H}), 8.68(\mathrm{~d}, J=4.8$ $\mathrm{Hz}, 2 \mathrm{H}), 8.23(\mathrm{~s}, 1 \mathrm{H}), 7.62(\mathrm{~d}, J=7.7 \mathrm{~Hz}, 1 \mathrm{H}), 7.37-7.33(\mathrm{~m}, 1 \mathrm{H}), 7.26-7.22(\mathrm{~m}, 1 \mathrm{H}), 7.02(\mathrm{t}, J=$ $4.8 \mathrm{~Hz}, 1 \mathrm{H}), 5.93(\mathrm{ddt}, J=15.3,8.1,1.4 \mathrm{~Hz}, 1 \mathrm{H}), 5.77(\mathrm{dt}, J=15.3,6.1 \mathrm{~Hz}, 1 \mathrm{H}), 4.58(\mathrm{~d}, J=8.1$ $\mathrm{Hz}, 1 \mathrm{H}), 3.72(\mathrm{~s}, 3 \mathrm{H}), 2.14-2.06(\mathrm{~m}, 2 \mathrm{H}), 1.01$ (t, $J=7.4 \mathrm{~Hz}, 3 \mathrm{H}) .{ }^{13} \mathrm{C} \mathrm{NMR}\left(75 \mathrm{MHz}, \mathrm{CDCl}_{3}\right) \delta$ $173.4,158.2,157.8,135.9,130.0,125.1,124.1,123.8,122.2,119.4,117.9,116.6,116.2,52.4$, 46.6, 25.6, 13.5. HRMS (ESI) calcd. for $\mathrm{C}_{21} \mathrm{H}_{20} \mathrm{~N}_{3} \mathrm{O}_{2}[\mathrm{M}+\mathrm{H}]^{+}:$322.1550, found: 322.1546 .

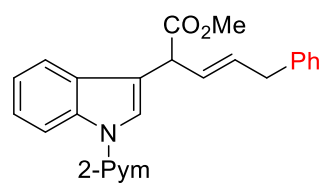




\section{Methyl (E)-5-phenyl-2-(1-(pyrimidin-2-yl)-1H-indol-3-yl)pent-3-enoate (3l):}

Obtained as colorless oil after column chromatography (petroleum ether/ethyl acetate $=20: 1$ to 10:1), $26 \mathrm{mg}$, 68\% yield. ${ }^{1} \mathrm{H}$ NMR $\left(300 \mathrm{MHz}, \mathrm{CDCl}_{3}\right) \delta 8.80(\mathrm{~d}, J=8.3 \mathrm{~Hz}, 1 \mathrm{H}), 8.66(\mathrm{~d}, J=4.8$ $\mathrm{Hz}, 2 \mathrm{H}), 8.24(\mathrm{~s}, 1 \mathrm{H}), 7.62(\mathrm{~d}, J=7.8 \mathrm{~Hz}, 1 \mathrm{H}), 7.38-7.32(\mathrm{~m}, 1 \mathrm{H}), 7.29-7.24(\mathrm{~m}, 3 \mathrm{H}), 7.21-7.15$ (m, 3H), 7.00 (t, $J=4.8 \mathrm{~Hz}, 1 \mathrm{H}), 6.07$ (ddt, $J=15.2 \mathrm{~Hz}, 8.0 \mathrm{~Hz}, 7.8 \mathrm{~Hz}, 1 \mathrm{H}), 5.91-5.81$ (m, 1H), $4.63(\mathrm{~d}, J=7.9 \mathrm{~Hz}, 1 \mathrm{H}), 3.72(\mathrm{~s}, 3 \mathrm{H}), 3.43(\mathrm{~d}, J=6.8 \mathrm{~Hz}, 2 \mathrm{H}) \cdot{ }^{13} \mathrm{C} \mathrm{NMR}\left(75 \mathrm{MHz}, \mathrm{CDCl}_{3}\right) \delta$ $173.1,158.2,157.7,140.1,135.9,132.6,129.9,128.7,128.5,127.8,126.2,124.1,123.9,122.2$, 119.4, 117.5, 116.6, 116.2, 52.4, 46.5, 38.9. HRMS (ESI) calcd. for $\mathrm{C}_{24} \mathrm{H}_{22} \mathrm{~N}_{3} \mathrm{O}_{2}[\mathrm{M}+\mathrm{H}]^{+}$: 384.1707, found: 384.1710 .

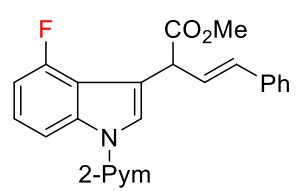

Methyl (E)-2-(4-fluoro-1-(pyrimidin-2-yl)-1H-indol-3-yl)-4-phenylbut-3-enoate (3m):

Obtained as colorless oil after column chromatography (petroleum ether/ethyl acetate $=20: 1$ to 10:1), $17 \mathrm{mg}, 45 \%$ yield. ${ }^{1} \mathrm{H}$ NMR $\left(300 \mathrm{MHz}, \mathrm{CDCl}_{3}\right) \delta 8.66(\mathrm{~d}, J=4.8 \mathrm{~Hz}, 2 \mathrm{H}), 8.60(\mathrm{~d}, J=8.3$ $\mathrm{Hz}, 1 \mathrm{H}), 8.26(\mathrm{~s}, 1 \mathrm{H}), 7.43-7.39(\mathrm{~m}, 2 \mathrm{H}), 7.33-7.27(\mathrm{~m}, 2 \mathrm{H}), 7.26-7.19(\mathrm{~m}, 2 \mathrm{H}), 7.03(\mathrm{t}, J=4.8 \mathrm{~Hz}$, $1 \mathrm{H}), 6.91$ (ddd, $J=10.7,8.0,0.6 \mathrm{~Hz}, 1 \mathrm{H}), 6.65-6.63(\mathrm{~m}, 2 \mathrm{H}), 4.99-4.96(\mathrm{~m}, 1 \mathrm{H}), 3.78(\mathrm{~s}, 3 \mathrm{H}) .{ }^{13} \mathrm{C}$ NMR $\left(75 \mathrm{MHz}, \mathrm{CDCl}_{3}\right) \delta 173.0,158.2,158.1,157.6,154.8,138.1,138.0,136.9,133.1,128.7$, 128.6, 127.8, 126.7, 126.3, 124.7, 124.6, 124.3, 118.6, 118.3, 116.7, 115.8, 115.7, 112.8, 112.7, 108.1, 107.8, 52.6, 47.1, 47.1. ${ }^{19} \mathrm{~F}$ NMR $\left(282 \mathrm{MHz}, \mathrm{CDCl}_{3}\right) \delta-123.0$. HRMS (ESI) calcd. for $\mathrm{C}_{23} \mathrm{H}_{19} \mathrm{FN}_{3} \mathrm{O}_{2}[\mathrm{M}+\mathrm{H}]^{+}: 388.1456$, found: 388.1452 .

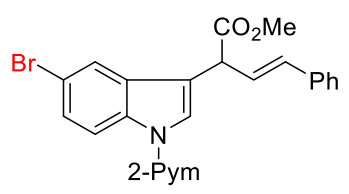

\section{Methyl (E)-2-(5-bromo-1-(pyrimidin-2-yl)-1H-indol-3-yl)-4-phenylbut-3-enoate (3n):}

Obtained as a white solid after column chromatography (petroleum ether/ethyl acetate $=20: 1$ to 10:1), $28 \mathrm{mg}, 63 \%$ yield, mp: $135-136{ }^{\circ} \mathrm{C} .{ }^{1} \mathrm{H}$ NMR $\left(400 \mathrm{MHz}, \mathrm{CDCl}_{3}\right) \delta 8.68(\mathrm{~d}, J=8.9 \mathrm{~Hz}, 1 \mathrm{H})$, $8.66(\mathrm{~d}, J=4.7 \mathrm{~Hz}, 2 \mathrm{H}), 8.31(\mathrm{~s}, 1 \mathrm{H}), 7.77-7.77(\mathrm{~m}, 1 \mathrm{H}), 7.44-7.39(\mathrm{~m}, 3 \mathrm{H}), 7.31(\mathrm{t}, J=7.3 \mathrm{~Hz}$, 2H), 7.25-7.21 (m, 1H), 7.04-7.02 (m, 1H), 6.58-6.69 (m, 2H), $4.72(\mathrm{~d}, J=6.8 \mathrm{~Hz}, 1 \mathrm{H}), 3.77(\mathrm{~s}$, $3 \mathrm{H}) .{ }^{13} \mathrm{C} \mathrm{NMR}\left(100 \mathrm{MHz}, \mathrm{CDCl}_{3}\right) \delta 172.5,158.2,157.4,136.6,134.5,133.1,131.6,128.7,127.9$, 127.0, 126.7, 125.6, 125.2, 121.9, 118.1, 116.6, 116.4, 115.7, 52.7, 46.5. HRMS (ESI) calcd. for $\mathrm{C}_{23} \mathrm{H}_{19} \mathrm{BrN}_{3} \mathrm{O}_{2}[\mathrm{M}+\mathrm{H}]^{+}:$448.0655, found: 448.0651 .

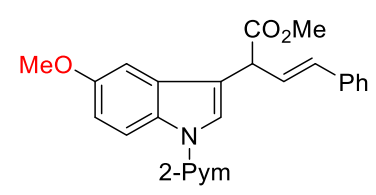

$\operatorname{Methyl}($ E)-2-(5-methoxy-1-(pyrimidin-2-yl)-1H-indol-3-yl)-4-phenylbut-3-enoate (3o):

Obtained as a white solid after column chromatography (petroleum ether/ethyl acetate $=20: 1$ to 10:1), $31 \mathrm{mg}, 77 \%$ yield, mp: $117-118{ }^{\circ} \mathrm{C} .{ }^{1} \mathrm{H}$ NMR $\left(400 \mathrm{MHz}, \mathrm{CDCl}_{3}\right) \delta 8.70(\mathrm{~d}, J=9.1 \mathrm{~Hz}, 1 \mathrm{H})$, 
$8.64(\mathrm{~d}, J=4.8 \mathrm{~Hz}, 2 \mathrm{H}), 8.27(\mathrm{~s}, 1 \mathrm{H}), 7.40(\mathrm{~d}, J=7.4 \mathrm{~Hz}, 2 \mathrm{H}), 7.30(\mathrm{t}, J=7.2 \mathrm{~Hz}, 2 \mathrm{H}), 7.25-7.21$ (m, 1H), 7.11-7.11 (m, 1H), 7.00-6.96 (m, 2H), $6.69(\mathrm{dd}, J=15.8,7.2 \mathrm{~Hz}, 1 \mathrm{H}), 6.62(\mathrm{~d}, J=15.8$ $\mathrm{Hz}, 1 \mathrm{H}), 4.75(\mathrm{~d}, J=7.2 \mathrm{~Hz}, 1 \mathrm{H}), 3.87(\mathrm{~s}, 3 \mathrm{H}), 3.76(\mathrm{~s}, 3 \mathrm{H}) .{ }^{13} \mathrm{C}$ NMR $\left(75 \mathrm{MHz}, \mathrm{CDCl}_{3}\right) \delta 172.8$, 158.2, 157.6, 155.6, 136.8, 132.9, 130.7, 130.7, 128.7, 127.8, 126.7, 125.9, 124.6, 117.5, 116.9, 116.1, 112.9, 102.0, 55.9, 52.6, 46.8. HRMS (ESI) calcd. for $\mathrm{C}_{24} \mathrm{H}_{22} \mathrm{~N}_{3} \mathrm{O}_{3}[\mathrm{M}+\mathrm{H}]^{+}$: 400.1656 , found: 400.1653 .

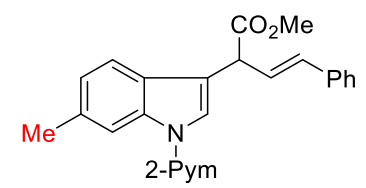

Methyl (E)-2-(6-methyl-1-(pyrimidin-2-yl)-1H-indol-3-yl)-4-phenylbut-3-enoate (3p):

Obtained as a white solid after column chromatography (petroleum ether/ethyl acetate $=20: 1$ to 10:1), $30 \mathrm{mg}, 79 \%$ yield, mp: $136-137{ }^{\circ} \mathrm{C} .{ }^{1} \mathrm{H}$ NMR (400 MHz, $\left.\mathrm{CDCl}_{3}\right) \delta 8.65-8.63(\mathrm{~m}, 3 \mathrm{H}), 8.22$ (s, 1H), $7.53(\mathrm{~d}, J=8.0 \mathrm{~Hz}, 1 \mathrm{H}), 7.40-7.38(\mathrm{~m}, 2 \mathrm{H}), 7.30-7.27(\mathrm{~m}, 2 \mathrm{H}), 7.22-7.19(\mathrm{~m}, 1 \mathrm{H})$, 7.09-7.07 (m, 1H), 6.97 (t, $J=4.8 \mathrm{~Hz}, 1 \mathrm{H}), 6.69(\mathrm{dd}, J=15.8,7.6 \mathrm{~Hz}, 1 \mathrm{H}), 6.61(\mathrm{~d}, J=15.8 \mathrm{~Hz}$, $1 \mathrm{H}), 4.76(\mathrm{~d}, J=7.6 \mathrm{~Hz}, 1 \mathrm{H}), 3.75(\mathrm{~s}, 3 \mathrm{H}), 2.53(\mathrm{~s}, 3 \mathrm{H}) .{ }^{13} \mathrm{C}$ NMR $\left(75 \mathrm{MHz}, \mathrm{CDCl}_{3}\right) \delta 172.8$, 158.1, 157.7, 136.8, 136.2, 134.1, 132.8, 128.6, 127.8, 127.7, 126.6, 126.1, 123.8, 123.5, 119.0, 117.1, 116.6, 116.1, 52.5, 46.9, 22.2. HRMS (ESI) calcd. for $\mathrm{C}_{24} \mathrm{H}_{22} \mathrm{~N}_{3} \mathrm{O}_{2}[\mathrm{M}+\mathrm{H}]^{+}:$:384.1707, found: 384.17108 .

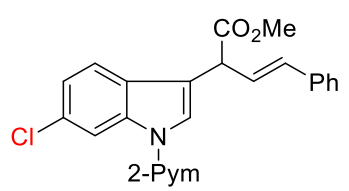

Methyl (E)-2-(6-chloro-1-(pyrimidin-2-yl)-1H-indol-3-yl)-4-phenylbut-3-enoate (3q):

Obtained as a white solid after column chromatography (petroleum ether/ethyl acetate $=20: 1$ to 10:1), $28 \mathrm{mg}, 69 \%$ yield, mp: $143-144{ }^{\circ} \mathrm{C} .{ }^{1} \mathrm{H}$ NMR $\left(400 \mathrm{MHz}, \mathrm{CDCl}_{3}\right) \delta 8.87(\mathrm{~d}, J=1.8 \mathrm{~Hz}, 1 \mathrm{H})$, $8.67(\mathrm{~d}, J=4.8 \mathrm{~Hz}, 2 \mathrm{H}), 8.28(\mathrm{~s}, 1 \mathrm{H}), 7.56(\mathrm{~d}, J=8.4 \mathrm{~Hz}, 1 \mathrm{H}), 7.39$ (d, $J=7.3 \mathrm{~Hz}, 2 \mathrm{H}), 7.30$ (t, $J$ $=7.2 \mathrm{~Hz}, 2 \mathrm{H}), 7.24-7.21(\mathrm{~m}, 2 \mathrm{H}), 7.03(\mathrm{t}, J=4.8 \mathrm{~Hz}, 1 \mathrm{H}), 6.67(\mathrm{dd}, J=15.8,7.2 \mathrm{~Hz}, 1 \mathrm{H}), 6.60(\mathrm{~d}$, $J=15.8 \mathrm{~Hz}, 1 \mathrm{H}), 4.75(\mathrm{~d}, J=7.2 \mathrm{~Hz}, 1 \mathrm{H}), 3.76(\mathrm{~s}, 3 \mathrm{H}) .{ }^{13} \mathrm{C} \mathrm{NMR}\left(100 \mathrm{MHz}, \mathrm{CDCl}_{3}\right) \delta 172.6$, 158.3, 157.3, 136.6, 136.1, 133.1, 130.1, 128.7, 128.4, 127.9, 126.7, 125.6, 124.6, 122.8, 120.1, 116.9, 116.8, 116.6, 52.6, 46.7. HRMS (ESI) calcd. for $\mathrm{C}_{23} \mathrm{H}_{19} \mathrm{ClN}_{3} \mathrm{O}_{2}[\mathrm{M}+\mathrm{H}]^{+}: 404.1106$, found: 404.1108.

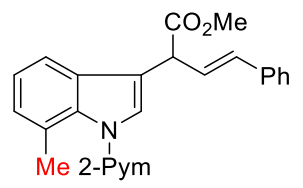

Methyl (E)-2-(7-methyl-1-phenyl-1H-indol-3-yl)-4-phenylbut-3-enoate (3r):

Obtained as colorless oil after column chromatography (petroleum ether/ethyl acetate $=20: 1$ to 10:1), $27 \mathrm{mg}, 70 \%$ yield. ${ }^{1} \mathrm{H}$ NMR (400 MHz, DMSO-d 6 ) $\delta 8.91(\mathrm{~d}, J=4.8 \mathrm{~Hz}, 2 \mathrm{H}), 7.87(\mathrm{~s}, 1 \mathrm{H})$, $7.55(\mathrm{~d}, J=7.6 \mathrm{~Hz}, 1 \mathrm{H}), 7.47-7.44(\mathrm{~m}, 3 \mathrm{H}), 7.33(\mathrm{t}, J=7.2 \mathrm{~Hz}, 2 \mathrm{H}), 7.25-7.22(\mathrm{~m}, 1 \mathrm{H}), 7.17(\mathrm{t}, J$ $=7.4 \mathrm{~Hz}, 1 \mathrm{H}), 7.11-7.09(\mathrm{~m}, 1 \mathrm{H}), 6.75(\mathrm{dd}, J=15.8,7.6 \mathrm{~Hz}, 1 \mathrm{H}), 6.68(\mathrm{~d}, J=15.8 \mathrm{~Hz}, 1 \mathrm{H}), 4.94$ 
(d, $J=7.6 \mathrm{~Hz}, 1 \mathrm{H}), 3.69$ (s, 3H), $2.30(\mathrm{~s}, 3 \mathrm{H}) .{ }^{13} \mathrm{C} \mathrm{NMR}\left(100 \mathrm{MHz}, \mathrm{CDCl}_{3}\right) \delta 172.9,158.3,158.0$, 136.8, 135.1, 132.7, 130.4, 128.6, 128.0, 127.7, 127.2, 126.6, 126.1, 124.6, 122.4, 117.5, 117.1, 117.0, 52.5, 46.6, 22.4. HRMS (ESI) calcd. for $\mathrm{C}_{24} \mathrm{H}_{22} \mathrm{~N}_{3} \mathrm{O}_{2}[\mathrm{M}+\mathrm{H}]^{+}: 384.1707$, found: 384.1702 .

\section{General procedure for Scheme 3}

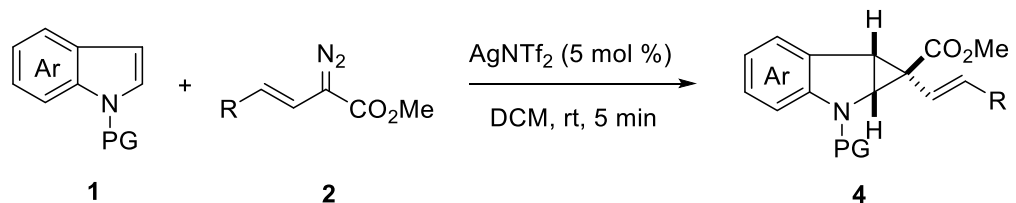

To a dry Schlenk tube was added $\mathbf{1}(0.1 \mathrm{mmol})$ and $\operatorname{AgNTf}_{2}(0.005 \mathrm{mmol})$. Then $\mathbf{2}(0.15 \mathrm{mmol})$ in $2 \mathrm{~mL}$ DCM was added in one portion and stirred at $\mathrm{rt}$ for $5 \mathrm{~min}$ under argon. The reaction mixture was concentrated under vacuum, the residue was purified by column chromatography (silica gel, petroleum ether/ethyl acetate $=10: 1$ to $5: 1$ ) to give 4 .

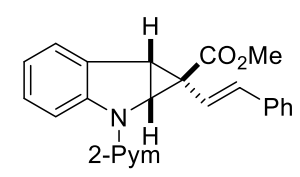

Methyl (E)-2-(pyrimidin-2-yl)-1-styryl-1,1a,2,6b-tetrahydrocyclopropa[b]indole-1-carboxylate

(4a):

Obtained as a white solid after column chromatography (petroleum ether/ethyl acetate $=10: 1$ to 5:1), $32 \mathrm{mg}, 86 \%$ yield, mp: $153-154{ }^{\circ} \mathrm{C} .{ }^{1} \mathrm{H}$ NMR $\left(400 \mathrm{MHz}, \mathrm{CDCl}_{3}\right) \delta 8.53(\mathrm{~d}, J=4.8 \mathrm{~Hz}, 2 \mathrm{H}$ ), $8.27(\mathrm{~d}, J=8.2 \mathrm{~Hz}, 1 \mathrm{H}), 7.45(\mathrm{~d}, J=7.3 \mathrm{~Hz}, 1 \mathrm{H}), 7.22(\mathrm{t}, J=7.6 \mathrm{~Hz}, 1 \mathrm{H}), 7.11-7.04(\mathrm{~m}, 3 \mathrm{H}), 7.00$ $(\mathrm{t}, J=7.4 \mathrm{~Hz}, 1 \mathrm{H}), 6.85-6.79(\mathrm{~m}, 3 \mathrm{H}), 6.16(\mathrm{~d}, J=16.2 \mathrm{~Hz}, 1 \mathrm{H}), 5.45(\mathrm{~d}, J=16.2 \mathrm{~Hz}, 1 \mathrm{H}), 5.26(\mathrm{~d}$, $J=6.7 \mathrm{~Hz}, 1 \mathrm{H}), 3.77$ (s, $3 \mathrm{H}), 3.66(\mathrm{~d}, J=6.7 \mathrm{~Hz}, 1 \mathrm{H}) .{ }^{13} \mathrm{C} \mathrm{NMR}\left(75 \mathrm{MHz}, \mathrm{CDCl}_{3}\right) \delta 173.9,159.6$, $157.8,143.7,138.0,137.1,128.8,128.3,128.2,127.4,126.2,125.5,121.9,117.8,115.9,113.3$, 52.7, 52.2, 35.7, 28.0. HRMS (ESI) calcd. for $\mathrm{C}_{23} \mathrm{H}_{20} \mathrm{~N}_{3} \mathrm{O}_{2}[\mathrm{M}+\mathrm{H}]^{+}: 370.1550$, found: 370.1546 .

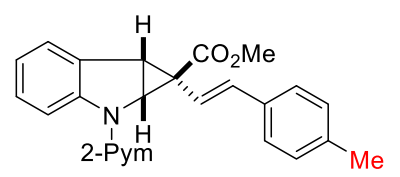

\section{Methyl}

(E)-1-(4-methylstyryl)-2-(pyrimidin-2-yl)-1,1a,2,6b-tetrahydrocyclopropa[b]indole-1-carboxylat $e(4 b)$ :

Obtained as a white solid after column chromatography (petroleum ether/ethyl acetate $=10: 1$ to 5:1), $31 \mathrm{mg}, 82 \%$ yield, mp: $135-136{ }^{\circ} \mathrm{C} .{ }^{1} \mathrm{H}$ NMR $\left(300 \mathrm{MHz}, \mathrm{CDCl}_{3}\right) \delta 8.52(\mathrm{~d}, J=4.8 \mathrm{~Hz}, 2 \mathrm{H})$, $8.27(\mathrm{~d}, J=8.2 \mathrm{~Hz}, 1 \mathrm{H}), 7.44(\mathrm{~d}, J=7.3 \mathrm{~Hz}, 1 \mathrm{H}), 7.23-7.18(\mathrm{~m}, 1 \mathrm{H}), 6.98(\mathrm{td}, J=7.4,0.8 \mathrm{~Hz}, 1 \mathrm{H})$, $6.89(\mathrm{~d}, J=8.0 \mathrm{~Hz}, 2 \mathrm{H}), 6.79(\mathrm{t}, J=4.8 \mathrm{~Hz}, 1 \mathrm{H}), 6.74(\mathrm{~d}, J=8.1 \mathrm{~Hz}, 2 \mathrm{H}), 6.12(\mathrm{~d}, J=16.2 \mathrm{~Hz}$, $1 \mathrm{H}), 5.40(\mathrm{~d}, J=16.2 \mathrm{~Hz}, 1 \mathrm{H}), 5.25(\mathrm{~d}, J=6.7 \mathrm{~Hz}, 1 \mathrm{H}), 3.76(\mathrm{~s}, 3 \mathrm{H}), 3.65(\mathrm{~d}, J=6.7 \mathrm{~Hz}, 1 \mathrm{H})$, 2.19 (s, 3H). ${ }^{13} \mathrm{C}$ NMR $\left(75 \mathrm{MHz}, \mathrm{CDCl}_{3}\right) \delta 174.0,159.6,157.8,143.7,137.9,137.2,134.3,129.0$, $128.8,128.1,126.1,125.4,121.8,116.6,115.9,113.2,52.6,52.2,35.6,28.0,21.2$. HRMS (ESI) calcd. for $\mathrm{C}_{24} \mathrm{H}_{22} \mathrm{~N}_{3} \mathrm{O}_{2}[\mathrm{M}+\mathrm{H}]^{+}: 384.1707$, found: 384.1704 . 


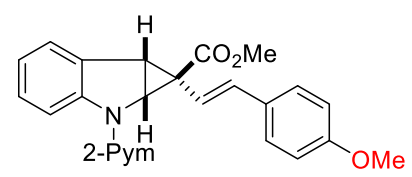

\section{Methyl}

(E)-1-(4-methoxystyryl)-2-(pyrimidin-2-yl)-1,1a,2,6b-tetrahydrocyclopropa[b]indole-1-carboxyl ate $(4 c)$ :

Obtained as a white solid after column chromatography (petroleum ether/ethyl acetate $=10: 1$ to 5:1), $32 \mathrm{mg}, 79 \%$ yield, mp: $153-154{ }^{\circ} \mathrm{C} .{ }^{1} \mathrm{H}$ NMR $\left(400 \mathrm{MHz}, \mathrm{CDCl}_{3}\right) \delta 8.54(\mathrm{~d}, J=4.8 \mathrm{~Hz}, 2 \mathrm{H})$, $8.27(\mathrm{~d}, J=8.2 \mathrm{~Hz}, 1 \mathrm{H}), 7.44(\mathrm{~d}, J=7.3 \mathrm{~Hz}, 1 \mathrm{H}), 7.23-7.19(\mathrm{~m}, 1 \mathrm{H}), 6.99$ (t, $J=7.4 \mathrm{~Hz}, 1 \mathrm{H})$, 6.82-6.77 (m, 3H), $6.63(\mathrm{~d}, J=8.7 \mathrm{~Hz}, 2 \mathrm{H}), 6.09(\mathrm{~d}, J=16.2 \mathrm{~Hz}, 1 \mathrm{H}), 5.30(\mathrm{~d}, J=16.2 \mathrm{~Hz}, 1 \mathrm{H})$, $5.25(\mathrm{~d}, J=6.7 \mathrm{~Hz}, 1 \mathrm{H}), 3.78(\mathrm{~s}, 3 \mathrm{H}), 3.69(\mathrm{~s}, 3 \mathrm{H}), 3.65(\mathrm{~d}, J=6.7 \mathrm{~Hz}, 1 \mathrm{H}) .{ }^{13} \mathrm{C}$ NMR $(75 \mathrm{MHz}$, $\left.\mathrm{CDCl}_{3}\right) \delta 174.2,159.7,159.1,157.8,143.8,137.5,130.0,128.9,128.1,127.4,125.5,121.8,115.9$, 115.4, 113.7, 113.2, 55.3, 52.7, 52.2, 35.7, 28.1. HRMS (ESI) calcd. for $\mathrm{C}_{24} \mathrm{H}_{22} \mathrm{~N}_{3} \mathrm{O}_{3}[\mathrm{M}+\mathrm{H}]^{+}$: 400.1656, found: 400.1658 .

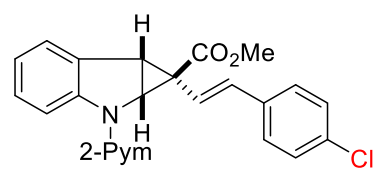

\section{Methyl}

(E)-1-(4-chlorostyryl)-2-(pyrimidin-2-yl)-1,1a,2,6b-tetrahydrocyclopropa[b]indole-1-carboxylate $(4 d)$ :

Obtained as a white solid after column chromatography (petroleum ether/ethyl acetate $=10: 1$ to 5:1), $30 \mathrm{mg}, 75 \%$ yield, mp: $157-158{ }^{\circ} \mathrm{C} .{ }^{1} \mathrm{H}$ NMR $\left(400 \mathrm{MHz}, \mathrm{CDCl}_{3}\right) \delta 8.55(\mathrm{~d}, J=4.8 \mathrm{~Hz}, 2 \mathrm{H})$, $8.26(\mathrm{~d}, J=8.2 \mathrm{~Hz}, 1 \mathrm{H}), 7.45(\mathrm{~d}, J=7.2 \mathrm{~Hz}, 1 \mathrm{H}), 7.25-7.21(\mathrm{~m}, 1 \mathrm{H}), 7.05(\mathrm{~d}, J=8.5 \mathrm{~Hz}, 2 \mathrm{H})$, $7.00(\mathrm{td}, J=7.4,0.7 \mathrm{~Hz}, 1 \mathrm{H}), 6.83(\mathrm{t}, J=4.8 \mathrm{~Hz}, 1 \mathrm{H}), 6.75(\mathrm{~d}, J=8.4 \mathrm{~Hz}, 2 \mathrm{H}), 6.10(\mathrm{~d}, J=16.2$ $\mathrm{Hz}, 1 \mathrm{H}), 5.42(\mathrm{~d}, J=16.2 \mathrm{~Hz}, 1 \mathrm{H}), 5.27(\mathrm{~d}, J=6.7 \mathrm{~Hz}, 1 \mathrm{H}), 3.79(\mathrm{~s}, 3 \mathrm{H}), 3.67(\mathrm{~d}, J=6.7 \mathrm{~Hz}, 1 \mathrm{H})$. ${ }^{13} \mathrm{C}$ NMR $\left(75 \mathrm{MHz}, \mathrm{CDCl}_{3}\right) \delta 173.7,159.6,157.9,143.7,136.8,135.6,133.1,128.7,128.5,128.2$, 127.4, 125.5, 121.9, 118.5, 115.9, 113.3, 52.7, 52.3, 35.7, 27.9. HRMS (ESI) calcd. for $\mathrm{C}_{23} \mathrm{H}_{19} \mathrm{ClN}_{3} \mathrm{O}_{2}[\mathrm{M}+\mathrm{H}]^{+}:$404.1160, found: 404.1156.

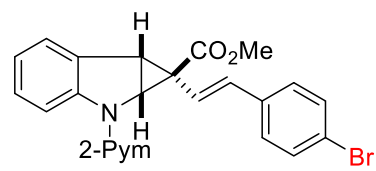

\section{Methyl}

(E)-1-(4-bromostyryl)-2-(pyrimidin-2-yl)-1,1a,2,6b-tetrahydrocyclopropa[b]indole-1-carboxylate $(4 e)$ :

Obtained as a white solid after column chromatography (petroleum ether/ethyl acetate $=10: 1$ to 5:1), $28 \mathrm{mg}, 62 \%$ yield, mp: $174-175{ }^{\circ} \mathrm{C} .{ }^{1} \mathrm{H}$ NMR $\left(400 \mathrm{MHz}, \mathrm{CDCl}_{3}\right) \delta 8.52(\mathrm{~d}, J=4.7 \mathrm{~Hz}, 2 \mathrm{H})$, $8.26(\mathrm{~d}, J=8.2 \mathrm{~Hz}, 1 \mathrm{H}), 7.44(\mathrm{~d}, J=7.4 \mathrm{~Hz}, 1 \mathrm{H}), 7.24-7.17$ (m, 3H), 6.99 (t, $J=7.4 \mathrm{~Hz}, 1 \mathrm{H}), 6.79$ (t, $J=4.7 \mathrm{~Hz}, 1 \mathrm{H}), 6.67(\mathrm{~d}, J=8.3 \mathrm{~Hz}, 2 \mathrm{H}), 6.08(\mathrm{~d}, J=16.1 \mathrm{~Hz}, 1 \mathrm{H}), 5.43(\mathrm{~d}, J=16.1 \mathrm{~Hz}, 1 \mathrm{H})$, $5.26(\mathrm{~d}, J=6.7 \mathrm{~Hz}, 1 \mathrm{H}), 3.77(\mathrm{~s}, 3 \mathrm{H}), 3.67(\mathrm{~d}, J=6.7 \mathrm{~Hz}, 1 \mathrm{H}) .{ }^{13} \mathrm{C} \mathrm{NMR}\left(75 \mathrm{MHz}, \mathrm{CDCl}_{3}\right) \delta$ 
173.6, 159.6, 157.8, 143.6, 136.8, 135.9, 131.4, 128.6, 128.2, 127.6, 125.5, 121.9, 121.2, 118.6, 115.8, 113.3, 52.7, 52.2, 35.6, 27.9. HRMS (ESI) calcd. for $\mathrm{C}_{23} \mathrm{H}_{19} \mathrm{BrN}_{3} \mathrm{O}_{2}[\mathrm{M}+\mathrm{H}]^{+}:$: 448.0655, found: 448.0659 .

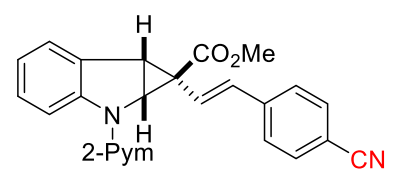

\section{Methyl}

(E)-1-(4-cyanostyryl)-2-(pyrimidin-2-yl)-1,1a,2,6b-tetrahydrocyclopropa[b]indole-1-carboxylate $(4 f):$

Obtained as a white solid after column chromatography (petroleum ether/ethyl acetate $=5: 1$ ), 26 mg, $65 \%$ yield, mp: $166-167^{\circ} \mathrm{C} .{ }^{1} \mathrm{H}$ NMR $\left(300 \mathrm{MHz}, \mathrm{CDCl}_{3}\right) \delta 8.54(\mathrm{~d}, J=4.8 \mathrm{~Hz}, 2 \mathrm{H}), 8.27(\mathrm{~d}, J$ $=8.2 \mathrm{~Hz}, 1 \mathrm{H}), 7.46(\mathrm{~d}, J=7.1 \mathrm{~Hz}, 1 \mathrm{H}), 7.34(\mathrm{~d}, J=8.3 \mathrm{~Hz}, 2 \mathrm{H}), 7.26-7.20(\mathrm{~m}, 1 \mathrm{H}), 7.01(\mathrm{td}, J=$ 7.4, $0.8 \mathrm{~Hz}, 1 \mathrm{H}), 6.88-6.81(\mathrm{~m}, 3 \mathrm{H}), 6.17(\mathrm{~d}, J=16.2 \mathrm{~Hz}, 1 \mathrm{H}), 5.58(\mathrm{~d}, J=16.2 \mathrm{~Hz}, 1 \mathrm{H}), 5.30(\mathrm{~d}$, $J=6.7 \mathrm{~Hz}, 1 \mathrm{H}), 3.79(\mathrm{~s}, 3 \mathrm{H}), 3.71(\mathrm{~d}, J=6.7 \mathrm{~Hz}, 1 \mathrm{H}) .{ }^{13} \mathrm{C}$ NMR $\left(100 \mathrm{MHz}, \mathrm{CDCl}_{3}\right) \delta 173.1$, $159.4,157.8,143.5,141.3,136.1,132.1,128.4,128.3,126.5,125.5,122.0,122.0,118.9,115.8$, 113.4, 110.5, 52.7, 52.4, 35.8, 27.7. HRMS (ESI) calcd. for $\mathrm{C}_{24} \mathrm{H}_{19} \mathrm{~N}_{4} \mathrm{O}_{2}[\mathrm{M}+\mathrm{H}]^{+}: 395.1503$, found: 395.1505 .

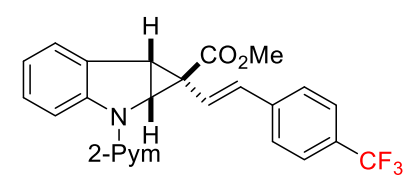

\section{Methyl}

(E)-2-(pyrimidin-2-yl)-1-(4-(trifluoromethyl)styryl)-1,1a,2,6b-tetrahydrocyclopropa[b]indole-1-c arboxylate (4g):

Obtained as a white solid after column chromatography (petroleum ether/ethyl acetate $=10: 1$ to 5:1), $31 \mathrm{mg}, 72 \%$ yield, mp: $166-167^{\circ} \mathrm{C} .{ }^{1} \mathrm{H}$ NMR $\left(400 \mathrm{MHz}, \mathrm{CDCl}_{3}\right) \delta 8.55(\mathrm{~d}, J=4.7 \mathrm{~Hz}, 2 \mathrm{H})$, $8.28(\mathrm{~d}, J=8.2 \mathrm{~Hz}, 1 \mathrm{H}), 7.46(\mathrm{~d}, J=7.4 \mathrm{~Hz}, 1 \mathrm{H}), 7.33(\mathrm{~d}, J=8.0 \mathrm{~Hz}, 2 \mathrm{H}), 7.24(\mathrm{t}, J=7.8 \mathrm{~Hz}, 1 \mathrm{H})$, $7.01(\mathrm{t}, J=7.4 \mathrm{~Hz}, 1 \mathrm{H}), 6.90(\mathrm{~d}, J=8.0 \mathrm{~Hz}, 2 \mathrm{H}), 6.83(\mathrm{t}, J=4.7 \mathrm{~Hz}, 1 \mathrm{H}), 6.19$ (d, $J=16.2 \mathrm{~Hz}$, $1 \mathrm{H}), 5.55(\mathrm{~d}, J=16.2 \mathrm{~Hz}, 1 \mathrm{H}), 5.29(\mathrm{~d}, J=6.7 \mathrm{~Hz}, 1 \mathrm{H}), 3.79(\mathrm{~s}, 3 \mathrm{H}), 3.70(\mathrm{~d}, J=6.7 \mathrm{~Hz}, 1 \mathrm{H}) .{ }^{13} \mathrm{C}$ NMR $\left(75 \mathrm{MHz}, \mathrm{CDCl}_{3}\right) \delta 173.5,159.6,157.9,143.7,140.5,136.6,129.4,129.0,128.6,128.3$, 126.3, 126.0, 125.6, 125.4, 125.3, 125.3, 125.2, 122.4, 122.0, 120.7, 115.9, 113.4, 52.8, 52.4, 35.8, 27.9. ${ }^{19} \mathrm{~F}$ NMR (282 MHz, $\left.\mathrm{CDCl}_{3}\right) \delta$-62.6. HRMS (ESI) calcd. for $\mathrm{C}_{24} \mathrm{H}_{19} \mathrm{~F}_{3} \mathrm{~N}_{3} \mathrm{O}_{2}[\mathrm{M}+\mathrm{H}]^{+}$: 438.1424, found: 438.1422 .

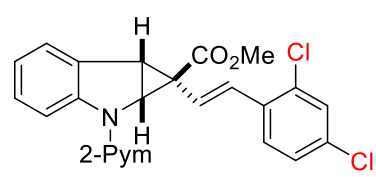

\section{Methyl}

(E)-1-(2,4-dichlorostyryl)-2-(pyrimidin-2-yl)-1,1a,2,6b-tetrahydrocyclopropa[b]indole-1-carboxy late (4h): 
Obtained as a white solid after column chromatography (petroleum ether/ethyl acetate $=10: 1$ to 5:1), $30 \mathrm{mg}, 69 \%$ yield, mp: $199-200{ }^{\circ} \mathrm{C} .{ }^{1} \mathrm{H}$ NMR $\left(400 \mathrm{MHz}, \mathrm{CDCl}_{3}\right) \delta 8.56(\mathrm{~d}, J=4.6 \mathrm{~Hz}, 2 \mathrm{H})$, $8.30(\mathrm{~d}, J=8.2 \mathrm{~Hz}, 1 \mathrm{H}), 7.44(\mathrm{~d}, J=7.3 \mathrm{~Hz}, 1 \mathrm{H}), 7.22(\mathrm{t}, J=8.0 \mathrm{~Hz}, 1 \mathrm{H}), 7.12(\mathrm{~s}, 1 \mathrm{H}), 7.00-6.92$ $(\mathrm{m}, 3 \mathrm{H}), 6.82(\mathrm{t}, J=4.7 \mathrm{~Hz}, 1 \mathrm{H}), 6.48(\mathrm{~d}, J=16.0 \mathrm{~Hz}, 1 \mathrm{H}), 5.44(\mathrm{~d}, J=16.0 \mathrm{~Hz}, 1 \mathrm{H}), 5.28(\mathrm{~d}, J=$ $6.7 \mathrm{~Hz}, 1 \mathrm{H}), 3.80(\mathrm{~s}, 3 \mathrm{H}), 3.67(\mathrm{~d}, J=6.7 \mathrm{~Hz}, 1 \mathrm{H}) .{ }^{13} \mathrm{C} \mathrm{NMR}\left(75 \mathrm{MHz}, \mathrm{CDCl}_{3}\right) \delta 173.6,159.6$, 157.9, 143.6, 133.8, 133.5, 133.4, 133.3, 129.1, 128.4, 128.3, 127.5, 127.0, 125.6, 122.0, 121.0, 115.9, 113.4, 52.8, 52.1, 35.8, 28.1. HRMS (ESI) calcd. for $\mathrm{C}_{23} \mathrm{H}_{18} \mathrm{Cl}_{2} \mathrm{~N}_{3} \mathrm{O}_{2}[\mathrm{M}+\mathrm{H}]^{+}$: 438.0771, found: 438.0768 .

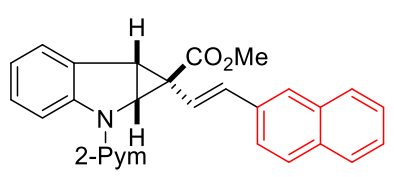

\section{Methyl}

(E)-1-(2-(naphthalen-2-yl)vinyl)-2-(pyrimidin-2-yl)-1,1a,2,6b-tetrahydrocyclopropa[b]indole-1-c arboxylate (4i):

Obtained as a white solid after column chromatography (petroleum ether/ethyl acetate $=10: 1$ to 5:1), $39 \mathrm{mg}, 92 \%$ yield, mp: $145-146{ }^{\circ} \mathrm{C} .{ }^{1} \mathrm{H}$ NMR $\left(300 \mathrm{MHz}, \mathrm{CDCl}_{3}\right) \delta 8.53(\mathrm{~d}, J=4.8 \mathrm{~Hz}, 2 \mathrm{H})$, $8.22(\mathrm{~d}, J=8.2 \mathrm{~Hz}, 1 \mathrm{H}), 7.66-7.59(\mathrm{~m}, 2 \mathrm{H}), 7.53(\mathrm{~d}, J=8.6 \mathrm{~Hz}, 1 \mathrm{H}), 7.47(\mathrm{~d}, J=7.4 \mathrm{~Hz}, 1 \mathrm{H})$, 7.37-7.29 (m, 2H), 7.23-7.18 (m, 2H), 7.05-6.98 (m, 2H), $6.80(\mathrm{t}, J=4.8 \mathrm{~Hz}, 1 \mathrm{H}), 6.32(\mathrm{~d}, J=$ $16.1 \mathrm{~Hz}, 1 \mathrm{H}), 5.57$ (d, $J=16.1 \mathrm{~Hz}, 1 \mathrm{H}), 5.30$ (d, $J=6.7 \mathrm{~Hz}, 1 \mathrm{H}), 3.79$ (s, $3 \mathrm{H}), 3.70$ (d, $J=6.7 \mathrm{~Hz}$, 1H). ${ }^{13} \mathrm{C}$ NMR $\left(75 \mathrm{MHz}, \mathrm{CDCl}_{3}\right) \delta 174.0,159.7,157.8,143.7,138.1,134.5,133.4,132.9,128.8$, 128.2, 128.0, 127.9, 127.6, 126.1, 126.0, 125.8, 125.5, 123.5, 121.9, 118.1, 115.9, 113.3, 52.7, 52.3, 35.8, 28.1. HRMS (ESI) calcd. for $\mathrm{C}_{27} \mathrm{H}_{22} \mathrm{~N}_{3} \mathrm{O}_{2}[\mathrm{M}+\mathrm{H}]^{+}$: 420.1707, found: 420.1710 .

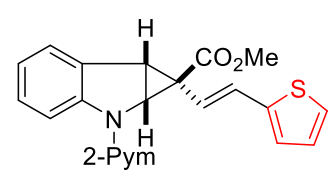

\section{Methyl}

(E)-2-(pyrimidin-2-yl)-1-(2-(thiophen-2-yl)vinyl)-1,1a,2,6b-tetrahydrocyclopropa[b]indole-1-car boxylate $(4 j)$ :

Obtained as a light yellow solid after column chromatography (petroleum ether/ethyl acetate $=$ 10:1 to 5:1), $23 \mathrm{mg}, 62 \%$ yield, mp: $123-124{ }^{\circ} \mathrm{C} .{ }^{1} \mathrm{H}$ NMR $\left(300 \mathrm{MHz}, \mathrm{CDCl}_{3}\right.$ ) $\delta 8.53$ (d, $J=4.8$ $\mathrm{Hz}, 2 \mathrm{H}), 8.29(\mathrm{~d}, J=8.3 \mathrm{~Hz}, 1 \mathrm{H}), 7.44(\mathrm{dd}, J=7.4,0.7 \mathrm{~Hz}, 1 \mathrm{H}), 7.26-7.20(\mathrm{~m}, 1 \mathrm{H}), 7.00(\mathrm{td}, J=$ 7.4, 1.0 Hz, 1H), $6.93(\mathrm{~d}, J=5.1 \mathrm{~Hz}, 1 \mathrm{H}), 6.80$ (t, $J=4.8 \mathrm{~Hz}, 1 \mathrm{H}), 6.74(\mathrm{dd}, J=5.1,3.5 \mathrm{~Hz}, 1 \mathrm{H})$, $6.48(\mathrm{~d}, J=3.5 \mathrm{~Hz}, 1 \mathrm{H}), 6.26(\mathrm{~d}, J=16.0 \mathrm{~Hz}, 1 \mathrm{H}), 5.31-5.23(\mathrm{~m}, 2 \mathrm{H}), 3.77(\mathrm{~s}, 3 \mathrm{H}), 3.66(\mathrm{~d}, J=$ $6.8 \mathrm{~Hz}, 1 \mathrm{H}) .{ }^{13} \mathrm{C}$ NMR $\left(75 \mathrm{MHz}, \mathrm{CDCl}_{3}\right) \delta 173.8,159.6,157.8,143.7,142.0,130.9,128.6,128.2$, 127.1, 125.5, 125.3, 124.1, 122.0, 117.2, 115.9, 113.3, 52.7, 52.5, 35.8, 28.0. HRMS (ESI) calcd. for $\mathrm{C}_{21} \mathrm{H}_{18} \mathrm{~N}_{3} \mathrm{O}_{2} \mathrm{~S}[\mathrm{M}+\mathrm{H}]^{+}: 376.1114$, found: 376.1117 .

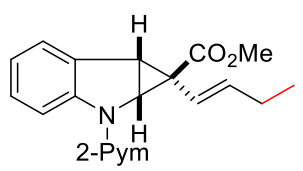




\section{Methyl}

(E)-1-(but-1-en-1-yl)-2-(pyrimidin-2-yl)-1,1a,2,6b-tetrahydrocyclopropa[b]indole-1-carboxylate (4k):

Obtained as colorless oil after column chromatography (petroleum ether/ethyl acetate $=10: 1$ to 5:1), $16 \mathrm{mg}, 51 \%$ yield. ${ }^{1} \mathrm{H}$ NMR $\left(400 \mathrm{MHz}, \mathrm{CDCl}_{3}\right) \delta 8.53(\mathrm{~d}, J=4.8 \mathrm{~Hz}, 2 \mathrm{H}), 8.32(\mathrm{~d}, J=8.2$ $\mathrm{Hz}, 1 \mathrm{H}), 7.38(\mathrm{~d}, J=7.3 \mathrm{~Hz}, 1 \mathrm{H}), 7.26-7.22(\mathrm{~m}, 1 \mathrm{H}), 6.97(\mathrm{td}, J=7.4,0.8 \mathrm{~Hz}, 1 \mathrm{H}), 6.79(\mathrm{t}, J=4.8$ $\mathrm{Hz}, 1 \mathrm{H}), 5.21(\mathrm{dt}, J=15.6,6.9 \mathrm{~Hz}, 1 \mathrm{H}), 5.14(\mathrm{~d}, J=6.8 \mathrm{~Hz}, 1 \mathrm{H}), 4.73(\mathrm{~d}, J=15.6 \mathrm{~Hz}, 1 \mathrm{H}), 3.75$ (s, 3H), $3.53(\mathrm{~d}, J=6.8 \mathrm{~Hz}, 1 \mathrm{H}), 1.65-1.58(\mathrm{~m}, 2 \mathrm{H}), 0.40(\mathrm{t}, J=7.4 \mathrm{~Hz}, 3 \mathrm{H}) .{ }^{13} \mathrm{C}$ NMR $(75 \mathrm{MHz}$, $\left.\mathrm{CDCl}_{3}\right) \delta 174.3,159.7,157.7,143.9,142.0,129.2,127.8,125.3,121.6,116.3,115.7,113.0,52.5$, 51.6, 35.2, 27.8, 25.7, 13.3. HRMS (ESI) calcd. for $\mathrm{C}_{19} \mathrm{H}_{20} \mathrm{~N}_{3} \mathrm{O}_{2}[\mathrm{M}+\mathrm{H}]^{+}: 322.1550$, found: 322.1555 .

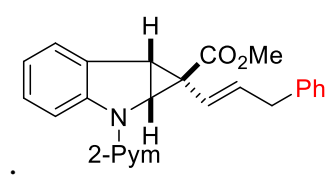

\section{Methyl}

(E)-1-(3-phenylprop-1-en-1-yl)-2-(pyrimidin-2-yl)-1,1a,2,6b-tetrahydrocyclopropa[b]indole-1-ca rboxylate $(4 l)$ :

Obtained as colorless oil after column chromatography (petroleum ether/ethyl acetate $=10: 1$ to 5:1), $28 \mathrm{mg}, 73 \%$ yield. ${ }^{1} \mathrm{H}$ NMR $\left(400 \mathrm{MHz}, \mathrm{CDCl}_{3}\right) \delta 8.46(\mathrm{~d}, J=4.7 \mathrm{~Hz}, 2 \mathrm{H}), 8.31(\mathrm{~d}, J=8.2$ $\mathrm{Hz}, 1 \mathrm{H}), 7.37(\mathrm{~d}, J=7.4 \mathrm{~Hz}, 1 \mathrm{H}), 7.26(\mathrm{t}, J=7.7 \mathrm{~Hz}, 1 \mathrm{H}), 7.07-7.06(\mathrm{~m}, 3 \mathrm{H}), 6.98(\mathrm{t}, J=7.4 \mathrm{~Hz}$, $1 \mathrm{H}), 6.72(\mathrm{t}, J=4.7 \mathrm{~Hz}, 1 \mathrm{H}), 6.59-6.57(\mathrm{~m}, 2 \mathrm{H}), 5.42-5.36(\mathrm{~m}, 1 \mathrm{H}), 5.14(\mathrm{~d}, J=6.8 \mathrm{~Hz}, 1 \mathrm{H}), 4.85$ $(\mathrm{d}, J=15.6 \mathrm{~Hz}, 1 \mathrm{H}), 3.77(\mathrm{~s}, 3 \mathrm{H}), 3.56(\mathrm{~d}, J=6.8 \mathrm{~Hz}, 1 \mathrm{H}), 3.06-2.94(\mathrm{~m}, 2 \mathrm{H}) .{ }^{13} \mathrm{C}$ NMR $(75 \mathrm{MHz}$, $\left.\mathrm{CDCl}_{3}\right) \delta 174.2,159.5,157.7,143.8,139.7,138.6,129.0,128.4,128.1,127.9,125.7,125.5,121.8$, 119.2, 116.0, 113.1, 52.6, 51.8, 38.9, 35.3, 27.9. HRMS (ESI) calcd. for $\mathrm{C}_{24} \mathrm{H}_{22} \mathrm{~N}_{3} \mathrm{O}_{2}[\mathrm{M}+\mathrm{H}]^{+}$: 384.1707, found: 384.1712 .

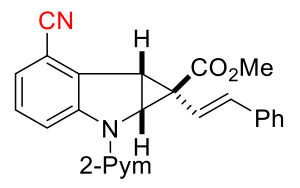

\section{Methyl}

(E)-6-cyano-2-(pyrimidin-2-yl)-1-styryl-1,1a,2,6b-tetrahydrocyclopropa[b]indole-1-carboxylate $(4 m)$ :

Obtained as a white solid after column chromatography (petroleum ether/ethyl acetate $=5: 1$ ), 22 mg, 55\% yield, mp: $107-108{ }^{\circ} \mathrm{C} .{ }^{1} \mathrm{H}$ NMR $\left(400 \mathrm{MHz}, \mathrm{CDCl}_{3}\right) \delta 8.59(\mathrm{~d}, J=4.8 \mathrm{~Hz}, 2 \mathrm{H}), 8.51(\mathrm{dd}$, $J=7.7,1.5 \mathrm{~Hz}, 1 \mathrm{H}), 7.29-7.23(\mathrm{~m}, 2 \mathrm{H}), 7.13-7.10(\mathrm{~m}, 3 \mathrm{H}), 6.92(\mathrm{t}, J=4.8 \mathrm{~Hz}, 1 \mathrm{H}), 6.87-6.85(\mathrm{~m}$, 2H), $6.14(\mathrm{~d}, J=16.1 \mathrm{~Hz}, 1 \mathrm{H}), 5.48(\mathrm{~d}, J=16.1 \mathrm{~Hz}, 1 \mathrm{H}), 5.33$ (d, $J=6.7 \mathrm{~Hz}, 1 \mathrm{H}), 3.87$ (d, $J=6.7$ $\mathrm{Hz}, 1 \mathrm{H}), 3.81$ (s, 3H). ${ }^{13} \mathrm{C} \mathrm{NMR}\left(75 \mathrm{MHz}, \mathrm{CDCl}_{3}\right) \delta 173.1,159.2,158.0,144.3,138.9,136.5$, 133.0, 128.9, 128.4, 127.9, 126.3, 124.9, 120.0, 117.6, 116.7, 114.3, 109.4, 53.0, 52.2, 34.2, 27.8. HRMS (ESI) calcd. for $\mathrm{C}_{24} \mathrm{H}_{19} \mathrm{~N}_{4} \mathrm{O}_{2}[\mathrm{M}+\mathrm{H}]^{+}:$395.1503, found: 395.1501 . 


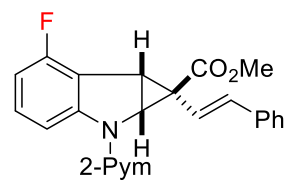

\section{Methyl}

(E)-6-fluoro-2-(pyrimidin-2-yl)-1-styryl-1,1a,2,6b-tetrahydrocyclopropa[b]indole-1-carboxylate (4n):

Obtained as colorless oil after column chromatography (petroleum ether/ethyl acetate $=10: 1$ to 5:1), $30 \mathrm{mg}, 78 \%$ yield. ${ }^{1} \mathrm{H}$ NMR $\left(300 \mathrm{MHz}, \mathrm{CDCl}_{3}\right) \delta 8.52(\mathrm{~d}, J=4.8 \mathrm{~Hz}, 2 \mathrm{H}), 8.05(\mathrm{~d}, J=8.3$ $\mathrm{Hz}, 1 \mathrm{H}), 7.17-7.11(\mathrm{~m}, 1 \mathrm{H}), 7.10-7.05(\mathrm{~m}, 3 \mathrm{H}), 6.88-6.85(\mathrm{~m}, 2 \mathrm{H}), 6.80(\mathrm{t}, J=4.8 \mathrm{~Hz}, 1 \mathrm{H})$, 6.72-6.67 (m, 1H), $6.19(\mathrm{~d}, J=16.1 \mathrm{~Hz}, 1 \mathrm{H}), 5.53(\mathrm{~d}, J=16.1 \mathrm{~Hz}, 1 \mathrm{H}), 5.26(\mathrm{~d}, J=6.8 \mathrm{~Hz}, 1 \mathrm{H})$, 3.77-3.76 (m, 4H). ${ }^{13} \mathrm{C}$ NMR $\left(75 \mathrm{MHz}, \mathrm{CDCl}_{3}\right) \delta 173.6,161.8,159.3,158.5,157.8,146.0,145.9$, 138.3, 136.8, 129.7, 129.6, 128.3, 127.6, 126.2, 117.3, 115.7, 115.5, 113.7, 111.8, 111.7, 108.6, 108.3, 52.7, 52.4, 31.6, 27.6. ${ }^{19} \mathrm{~F}$ NMR $\left(282 \mathrm{MHz}, \mathrm{CDCl}_{3}\right) \delta$-120.9. HRMS (ESI) calcd. for $\mathrm{C}_{23} \mathrm{H}_{19} \mathrm{FN}_{3} \mathrm{O}_{2}[\mathrm{M}+\mathrm{H}]^{+}:$388.1456, found: 388.1452 .

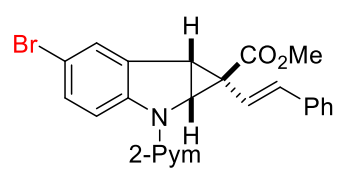

\section{Methyl}

(E)-5-bromo-2-(pyrimidin-2-yl)-1-styryl-1,1a,2,6b-tetrahydrocyclopropa[b]indole-1-carboxylate (4o):

Obtained as a white solid after column chromatography (petroleum ether/ethyl acetate $=10: 1$ to 5:1), $34 \mathrm{mg}, 75 \%$ yield, mp: $163-164{ }^{\circ} \mathrm{C} .{ }^{1} \mathrm{H}$ NMR $\left(400 \mathrm{MHz}, \mathrm{CDCl}_{3}\right) \delta 8.53(\mathrm{~d}, J=4.8 \mathrm{~Hz}, 2 \mathrm{H})$, $8.15(\mathrm{~d}, J=8.8 \mathrm{~Hz}, 1 \mathrm{H}), 7.55-7.52(\mathrm{~m}, 1 \mathrm{H}), 7.30(\mathrm{dd}, J=8.7,1.9 \mathrm{~Hz}, 1 \mathrm{H}), 7.14-7.07(\mathrm{~m}, 3 \mathrm{H})$, 6.88-6.86 (m, 2H), 6.83 (t, $J=4.8 \mathrm{~Hz}, 1 \mathrm{H}), 6.16(\mathrm{~d}, J=16.2 \mathrm{~Hz}, 1 \mathrm{H}), 5.46(\mathrm{~d}, J=16.2 \mathrm{~Hz}, 1 \mathrm{H})$, $5.25(\mathrm{~d}, J=6.7 \mathrm{~Hz}, 1 \mathrm{H}), 3.77(\mathrm{~s}, 3 \mathrm{H}), 3.60(\mathrm{~d}, J=6.7 \mathrm{~Hz}, 1 \mathrm{H}) .{ }^{13} \mathrm{C}$ NMR $\left(100 \mathrm{MHz}, \mathrm{CDCl}_{3}\right) \delta$ 173.6, 159.4, 157.9, 142.8, 138.4, 136.8, 131.0, 130.9, 128.4, 128.3, 127.7, 126.2, 117.3, 117.2, 114.0, 113.6, 52.8, 52.3, 35.0, 27.9. HRMS (ESI) calcd. for $\mathrm{C}_{23} \mathrm{H}_{19} \mathrm{BrN}_{3} \mathrm{O}_{2}[\mathrm{M}+\mathrm{H}]^{+}$: 448.0655, found: 448.0659

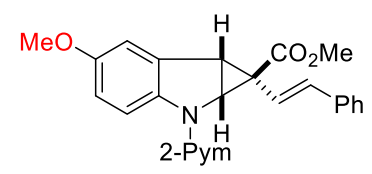

\section{Methyl}

(E)-5-methoxy-2-(pyrimidin-2-yl)-1-styryl-1,1a,2,6b-tetrahydrocyclopropa[b]indole-1-carboxylat $e(4 p)$ :

Obtained as a white solid after column chromatography (petroleum ether/ethyl acetate $=10: 1$ to 5:1), $29 \mathrm{mg}, 73 \%$ yield, mp: $154-155^{\circ} \mathrm{C} .{ }^{1} \mathrm{H}$ NMR $\left(400 \mathrm{MHz}, \mathrm{CDCl}_{3}\right) \delta 8.51(\mathrm{~d}, J=4.7 \mathrm{~Hz}, 2 \mathrm{H})$, $8.18(\mathrm{~d}, J=8.9 \mathrm{~Hz}, 1 \mathrm{H}), 7.13-7.08(\mathrm{~m}, 3 \mathrm{H}), 7.03(\mathrm{~d}, J=2.6 \mathrm{~Hz}, 1 \mathrm{H}), 6.88-6.86(\mathrm{~m}, 2 \mathrm{H}), 6.79-6.76$ $(\mathrm{m}, 2 \mathrm{H}), 6.19(\mathrm{~d}, J=16.2 \mathrm{~Hz}, 1 \mathrm{H}), 5.45(\mathrm{~d}, J=16.2 \mathrm{~Hz}, 1 \mathrm{H}), 5.25(\mathrm{~d}, J=6.7 \mathrm{~Hz}, 1 \mathrm{H}), 3.81(\mathrm{~s}$, $3 \mathrm{H}), 3.78(\mathrm{~s}, 3 \mathrm{H}), 3.62(\mathrm{~d}, J=6.7 \mathrm{~Hz}, 1 \mathrm{H}) .{ }^{13} \mathrm{C} \mathrm{NMR}\left(75 \mathrm{MHz}, \mathrm{CDCl}_{3}\right) \delta 173.9,159.5,157.8$, 
155.1, 138.0, 137.7, 137.2, 130.0 128.3, 127.5, 126.3, 117.7, 116.6, 113.4, 112.8, 111.3, 55.9, 52.7, 52.5, 35.8, 28.6. HRMS (ESI) calcd. for $\mathrm{C}_{24} \mathrm{H}_{22} \mathrm{~N}_{3} \mathrm{O}_{3}[\mathrm{M}+\mathrm{H}]^{+}: 400.1656$, found: 400.1653 .

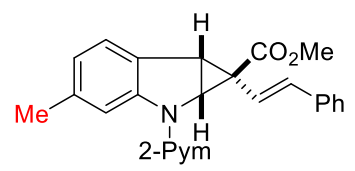

\section{Methyl}

(E)-4-methyl-2-(pyrimidin-2-yl)-1-styryl-1,1a,2,6b-tetrahydrocyclopropa[b]indole-1-carboxylate

$(4 q)$ :

Obtained as a white solid after column chromatography (petroleum ether/ethyl acetate $=10: 1$ to 5:1), $33 \mathrm{mg}, 85 \%$ yield, mp: $96-97{ }^{\circ} \mathrm{C} .{ }^{1} \mathrm{H}$ NMR $\left(400 \mathrm{MHz}, \mathrm{CDCl}_{3}\right) \delta 8.53(\mathrm{~d}, J=4.8 \mathrm{~Hz}, 2 \mathrm{H})$, $8.11(\mathrm{~s}, 1 \mathrm{H}), 7.31(\mathrm{~d}, J=7.6 \mathrm{~Hz}, 1 \mathrm{H}), 7.12-7.04(\mathrm{~m}, 3 \mathrm{H}), 6.87-6.85(\mathrm{~m}, 2 \mathrm{H}), 6.81-6.77(\mathrm{~m}, 2 \mathrm{H})$, $6.18(\mathrm{~d}, J=16.2 \mathrm{~Hz}, 1 \mathrm{H}), 5.46(\mathrm{~d}, J=16.2 \mathrm{~Hz}, 1 \mathrm{H}), 5.24(\mathrm{~d}, J=6.8 \mathrm{~Hz}, 1 \mathrm{H}), 3.76(\mathrm{~s}, 3 \mathrm{H}), 3.61(\mathrm{~d}$, $J=6.8 \mathrm{~Hz}, 1 \mathrm{H}), 2.33$ (s, 3H). ${ }^{13} \mathrm{C}$ NMR $\left(75 \mathrm{MHz}, \mathrm{CDCl}_{3}\right) \delta 173.9,159.6,157.8,143.9,138.1$, 137.9, 137.2, 128.3, 127.4, 126.2, 125.9, 125.1, 122.7, 117.9, 116.5, 113.1, 52.6, 52.5, 35.5, 28.3, 22.1. HRMS (ESI) calcd. for $\mathrm{C}_{24} \mathrm{H}_{22} \mathrm{~N}_{3} \mathrm{O}_{2}[\mathrm{M}+\mathrm{H}]^{+}: 384.1707$, found: 384.1703 .

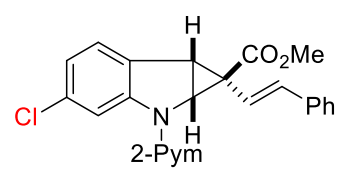

\section{Methyl}

(E)-4-chloro-2-(pyrimidin-2-yl)-1-styryl-1,1a,2,6b-tetrahydrocyclopropa[b]indole-1-carboxylate (4r):

Obtained as a white solid after column chromatography (petroleum ether/ethyl acetate $=10: 1$ to 5:1), $32 \mathrm{mg}, 78 \%$ yield, mp: $168-169^{\circ} \mathrm{C} .{ }^{1} \mathrm{H}$ NMR $\left(400 \mathrm{MHz}, \mathrm{CDCl}_{3}\right) \delta 8.54(\mathrm{~d}, J=4.8 \mathrm{~Hz}, 2 \mathrm{H})$, $8.33(\mathrm{~d}, J=1.7 \mathrm{~Hz}, 1 \mathrm{H}), 7.33(\mathrm{~d}, J=8.0 \mathrm{~Hz}, 1 \mathrm{H}), 7.14-7.08(\mathrm{~m}, 3 \mathrm{H}), 6.96(\mathrm{dd}, J=8.0,1.9 \mathrm{~Hz}$, $1 \mathrm{H}), 6.89-6.85(\mathrm{~m}, 2 \mathrm{H}), 6.83(\mathrm{t}, J=4.8 \mathrm{~Hz}, 1 \mathrm{H}), 6.16(\mathrm{~d}, J=16.2 \mathrm{~Hz}, 1 \mathrm{H}), 5.46(\mathrm{~d}, J=16.2 \mathrm{~Hz}$, $1 \mathrm{H}), 5.25(\mathrm{~d}, J=6.7 \mathrm{~Hz}, 1 \mathrm{H}), 3.76(\mathrm{~s}, 3 \mathrm{H}), 3.59$ (d, $J=6.7 \mathrm{~Hz}, 1 \mathrm{H}) .{ }^{13} \mathrm{C} \mathrm{NMR}\left(100 \mathrm{MHz}, \mathrm{CDCl}_{3}\right)$ $\delta 173.6,159.3,157.9,144.6,138.4,136.8,133.7,128.4,127.6,127.4,126.2,126.0,121.8,117.3$, 116.2, 113.7, 52.8, 52.5, 35.0, 27.9. HRMS (ESI) calcd. for $\mathrm{C}_{23} \mathrm{H}_{19} \mathrm{ClN}_{3} \mathrm{O}_{2}[\mathrm{M}+\mathrm{H}]^{+}:$: 404.1160, found: 404.1165 .

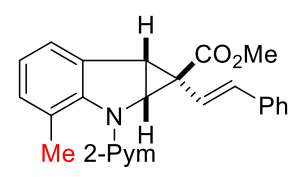

\section{Methyl}

(E)-3-methyl-2-(pyrimidin-2-yl)-1-styryl-1,1a,2,6b-tetrahydrocyclopropa[b]indole-1-carboxylate $(4 s):$

Obtained as colorless oil after column chromatography (petroleum ether/ethyl acetate $=10: 1$ to 5:1), $27 \mathrm{mg}, 80 \%$ yield. ${ }^{1} \mathrm{H}$ NMR (400 MHz, $\left.\mathrm{CDCl}_{3}\right) \delta 8.54(\mathrm{~d}, J=4.8 \mathrm{~Hz}, 2 \mathrm{H}), 7.32-7.30(\mathrm{~m}, 1 \mathrm{H})$, 7.14-7.09 (m, 3H), 7.03-6.97 (m, 2H), 6.92-6.90 (m, 2H), $6.86(\mathrm{t}, J=4.8 \mathrm{~Hz}, 1 \mathrm{H}), 6.39(\mathrm{~d}, J=$ 
$16.1 \mathrm{~Hz}, 1 \mathrm{H}), 5.35(\mathrm{~d}, J=16.1 \mathrm{~Hz}, 1 \mathrm{H}), 5.22(\mathrm{~d}, J=6.3 \mathrm{~Hz}, 1 \mathrm{H}), 3.75(\mathrm{~s}, 3 \mathrm{H}), 3.61(\mathrm{~d}, J=6.3 \mathrm{~Hz}$, 1H), $1.98(\mathrm{~s}, 3 \mathrm{H}) .{ }^{13} \mathrm{C}$ NMR $\left(75 \mathrm{MHz}, \mathrm{CDCl}_{3}\right) \delta 173.9,160.4,157.9,141.4,137.5,137.3,130.6$, $129.9,128.3,127.4,127.4,126.2,123.4,122.9,117.8,114.4,53.9,52.6,36.2,28.3,21.6$. HRMS (ESI) calcd. for $\mathrm{C}_{24} \mathrm{H}_{22} \mathrm{~N}_{3} \mathrm{O}_{2}[\mathrm{M}+\mathrm{H}]^{+}: 384.1707$, found: 384.1712 .

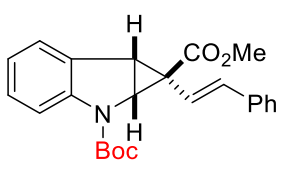

\section{2-(tert-butyl) 1-methyl (E)-1-styryl-1a,6b-dihydrocyclopropa[b]indole-1,2(1H)-dicarboxylate}

$(4 t)$ :

Obtained as light green oil after column chromatography (petroleum ether/ethyl acetate $=10: 1$ to 5:1), $16 \mathrm{mg}, 41 \%$ yield. ${ }^{1} \mathrm{H}$ NMR $\left(300 \mathrm{MHz}, \mathrm{CDCl}_{3}\right) \delta 7.71-7.73(\mathrm{~m}, 0.51 \mathrm{H}), 7.38(\mathrm{~d}, J=7.4 \mathrm{~Hz}$, 1H), 7.29-7.32 (m, 0.49H), 7.21-7.11 (m, 4H), 7.04-6.94 (m, 3H), $6.34(\mathrm{~d}, J=16.2 \mathrm{~Hz}, 1 \mathrm{H}), 5.46$ $(\mathrm{d}, J=16.2 \mathrm{~Hz}, 1 \mathrm{H}), 4.79(\mathrm{~d}, J=29.0 \mathrm{~Hz}, 1 \mathrm{H}), 3.75(\mathrm{~s}, 3 \mathrm{H}), 3.52$ (d, $J=6.8 \mathrm{~Hz}, 1 \mathrm{H}), 1.59$ (s, 9H). ${ }^{13} \mathrm{C}$ NMR $\left(75 \mathrm{MHz}, \mathrm{CDCl}_{3}\right) \delta 173.4,153.0,152.0,143.2,142.1,138.1,136.9,128.4,127.6,126.2$, $125.7,125.3,122.5,117.2,114.9,82.7,81.8,52.7,50.9,35.7,34.8,28.4,27.9$. HRMS (ESI) calcd. for $\mathrm{C}_{24} \mathrm{H}_{26} \mathrm{NO}_{4}[\mathrm{M}+\mathrm{H}]^{+}:$392.1856, found: 392.1858 .

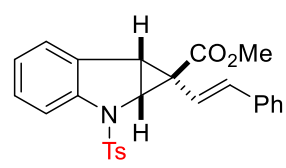

Methyl (E)-1-styryl-2-tosyl-1,1a,2,6b-tetrahydrocyclopropa[b]indole-1-carboxylate (4u):

Obtained as light green oil after column chromatography (petroleum ether/ethyl acetate $=10: 1$ to 5:1), $27 \mathrm{mg}, 60 \%$ yield. ${ }^{1} \mathrm{H}$ NMR (300 MHz, $\left.\mathrm{CDCl}_{3}\right) \delta 7.73-7.70(\mathrm{~m}, 2 \mathrm{H}), 7.49(\mathrm{~d}, J=8.2 \mathrm{~Hz}, 1 \mathrm{H})$, 7.36-7.33 (m, 1H), 7.19-7.11 (m, 6H), 7.00 (td, $J=7.5,1.0 \mathrm{~Hz}, 1 \mathrm{H}), 6.90-6.87(\mathrm{~m}, 2 \mathrm{H}), 6.30(\mathrm{~d}, J$ $=16.2 \mathrm{~Hz}, 1 \mathrm{H}), 5.26(\mathrm{~d}, J=16.2 \mathrm{~Hz}, 1 \mathrm{H}), 4.83(\mathrm{~d}, J=6.7 \mathrm{~Hz}, 1 \mathrm{H}), 3.74(\mathrm{~s}, 3 \mathrm{H}), 3.50(\mathrm{~d}, J=6.7$ $\mathrm{Hz}, 1 \mathrm{H}), 2.32$ (s, 3H). ${ }^{13} \mathrm{C}$ NMR $\left(75 \mathrm{MHz}, \mathrm{CDCl}_{3}\right) \delta 172.9,144.6,142.4,138.2,136.9,135.8$, 123.0, 128.7, 128.4, 128.3, 127.6, 127.0, 126.3, 125.9, 123.5, 116.8, 114.0, 53.2, 52.9, 35.4, 26.9, 21.7. HRMS (ESI) calcd. for $\mathrm{C}_{26} \mathrm{H}_{24} \mathrm{NO}_{4} \mathrm{~S}[\mathrm{M}+\mathrm{H}]^{+}: 446.1421$, found: 446.1424 .

\section{Further exploration for Scheme 4}

\section{Scheme 4-a}
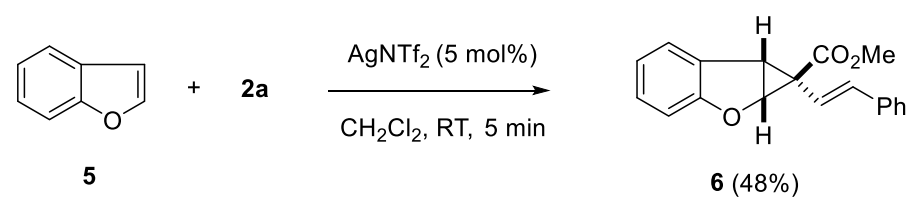

To a dry Schlenk tube was added $5(11.8 \mathrm{mg}, 0.1 \mathrm{mmol})$ and $\mathrm{AgNTf}_{2}(1.9 \mathrm{mg}, 0.005 \mathrm{mmol})$. Then 2a (30.3 mg, $0.15 \mathrm{mmol})$ in $2 \mathrm{~mL}$ DCM was added in one portion and stirred at $\mathrm{rt}$ for $5 \mathrm{~min}$ under argon. The reaction mixture was concentrated under vacuum, the residue was purified by column chromatography (silica gel, petroleum ether/ethyl acetate $=20: 1$ to 10:1) to give $\mathbf{6}$ as colorless oil, $14 \mathrm{mg}, 48 \%$ yield. ${ }^{1} \mathrm{H}$ NMR $\left(400 \mathrm{MHz}, \mathrm{CDCl}_{3}\right) \delta 7.38(\mathrm{~d}, J=7.4 \mathrm{~Hz}, 1 \mathrm{H}), 7.19-7.12(\mathrm{~m}, 3 \mathrm{H})$, 7.10-7.04 (m, 3H), 6.89 (t, $J=7.5 \mathrm{~Hz}, 1 \mathrm{H}), 6.75(\mathrm{~d}, J=8.1 \mathrm{~Hz}, 1 \mathrm{H}), 6.47(\mathrm{~d}, J=16.2 \mathrm{~Hz}, 1 \mathrm{H})$, $5.56(\mathrm{~d}, J=16.2 \mathrm{~Hz}, 1 \mathrm{H}), 5.24(\mathrm{~d}, J=5.5 \mathrm{~Hz}, 1 \mathrm{H}), 3.73(\mathrm{~s}, 3 \mathrm{H}), 3.58(\mathrm{~d}, J=5.5 \mathrm{~Hz}, 1 \mathrm{H}) .{ }^{13} \mathrm{C}$ 
NMR $\left(100 \mathrm{MHz}, \mathrm{CDCl}_{3}\right) \delta 173.2,160.3,138.0,137.0,128.5,128.4,127.6,126.3,125.6,125.2$, 121.4, 116.6, 110.0, 71.1, 52.7, 37.8, 26.9. HRMS (ESI) calcd. for $\mathrm{C}_{19} \mathrm{H}_{17} \mathrm{O}_{3}[\mathrm{M}+\mathrm{H}]^{+}:$293.1172, found: 293.1175 .

\section{Scheme 4-b}

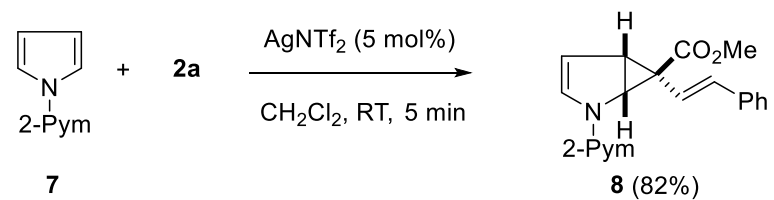

To a dry Schlenk tube was added $7(14.5 \mathrm{mg}, 0.1 \mathrm{mmol})$ and AgNTf $_{2}(1.9 \mathrm{mg}, 0.005 \mathrm{mmol})$. Then 2a (30.3 $\mathrm{mg}, 0.15 \mathrm{mmol})$ in $2 \mathrm{~mL}$ DCM was added in one portion and stirred at $\mathrm{rt}$ for 5 min under argon. The reaction mixture was concentrated under vacuum, the residue was purified by column chromatography (silica gel, petroleum ether/ethyl acetate $=10: 1$ to 5:1) to give $\mathbf{8}$ as a white solid, $20 \mathrm{mg}, 82 \%$ yield, mp: $128-129{ }^{\circ} \mathrm{C} .{ }^{1} \mathrm{H}$ NMR $\left(400 \mathrm{MHz}, \mathrm{CDCl}_{3}\right) \delta 8.40(\mathrm{~d}, J=4.7 \mathrm{~Hz}, 2 \mathrm{H})$, 7.33-7.24 (m, 3H), 7.21-7.18 (m, 2H), $6.68(\mathrm{dd}, J=6.1,2.6 \mathrm{~Hz}, 1 \mathrm{H}), 6.63-6.61(\mathrm{~m}, 2 \mathrm{H}), 5.61(\mathrm{dd}$, $J=6.0,2.5 \mathrm{~Hz}, 1 \mathrm{H}), 5.47-5.46(\mathrm{~m}, 1 \mathrm{H}), 5.33-5.29(\mathrm{~m}, 1 \mathrm{H}), 4.21(\mathrm{dd}, J=5.4,2.6 \mathrm{~Hz}, 1 \mathrm{H}), 3.82(\mathrm{~s}$, 3H). ${ }^{13} \mathrm{C}$ NMR $\left(100 \mathrm{MHz}, \mathrm{CDCl}_{3}\right) \delta 166.2,160.2,158.2,139.7,139.3,137.6,136.8,129.0,128.7$, 128.4, 127.3, 111.6, 62.7, 56.3, 52.1, 40.8. HRMS (ESI) calcd. for $\mathrm{C}_{19} \mathrm{H}_{18} \mathrm{~N}_{3} \mathrm{O}_{2}[\mathrm{M}+\mathrm{H}]^{+}: 320.1394$, found: 320.1390 .

\section{Scheme 4-c}

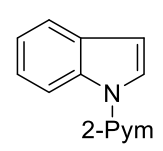

$1 \mathrm{a}$

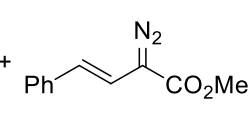

$2 a$

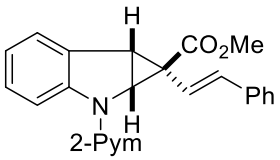

$4 a$

To a dry $50 \mathrm{ml}$ flask was added 1a (195.2 mg, $1.0 \mathrm{mmol})$ and $\operatorname{AgNTf}_{2}(19.4 \mathrm{mg}, 0.05 \mathrm{mmol})$. Then 2a (303 mg, $1.5 \mathrm{mmol})$ in $20 \mathrm{~mL}$ DCM was added in one portion and stirred at $\mathrm{rt}$ for 10 min under argon. The reaction mixture was concentrated under vacuum, the residue was purified by column chromatography (silica gel, petroleum ether/ethyl acetate $=10: 1$ to $5: 1$ ) to give $\mathbf{4 a}, 317 \mathrm{mg}, 86 \%$ yield.

\section{Scheme 4-d}
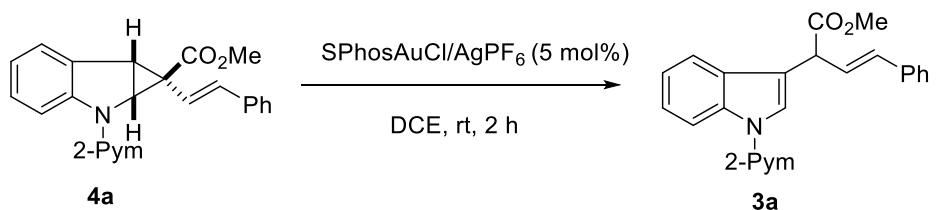

$2 \mathrm{~h}, 16 \%$ yield

$22 \mathrm{~h}, 98 \%$ yield

To a dry Schlenk tube was added SPhosAuCl (3.2 mg, $0.005 \mathrm{mmol}), \mathrm{AgPF}_{6}(1.3 \mathrm{mg}, 0.005 \mathrm{mmol})$ and DCE $(1 \mathrm{~mL})$. The mixture was stirred at $\mathrm{rt}$ for $0.5 \mathrm{~h}$ under an argon atmosphere, then $4 \mathbf{a}(36.9$ $\mathrm{mg}, 0.1 \mathrm{mmol}$ ) was added. The mixture was stirred at $\mathrm{rt}$ under argon atmosphere for $22 \mathrm{~h}$. The reaction mixture was concentrated under vacuum, the residue was purified by column chromatography (silica gel, petroleum ether/ethyl acetate $=10: 1$ ) to give $\mathbf{3 a}, 36 \mathrm{mg}, 98 \%$ yield. 


\section{Scheme 4-e}
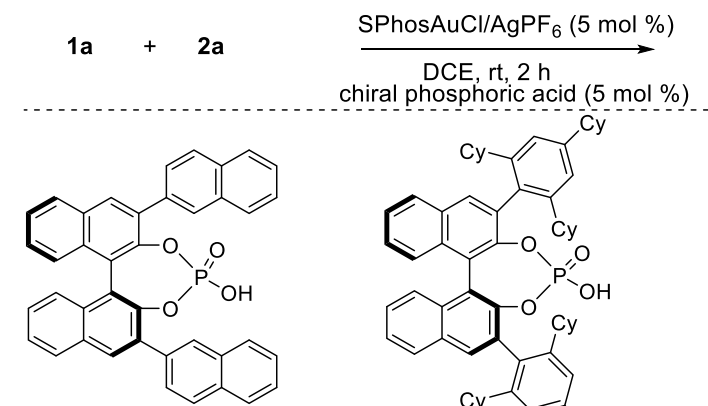

3a

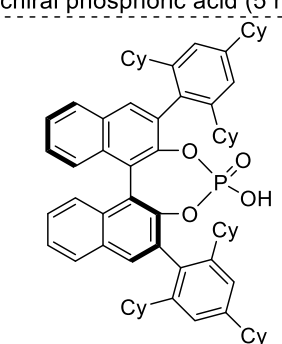

$67 \%$ yield, $12 \%$ ee

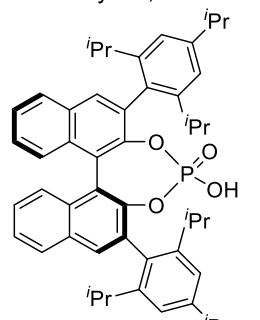

$65 \%$ yield, $11 \%$ ee
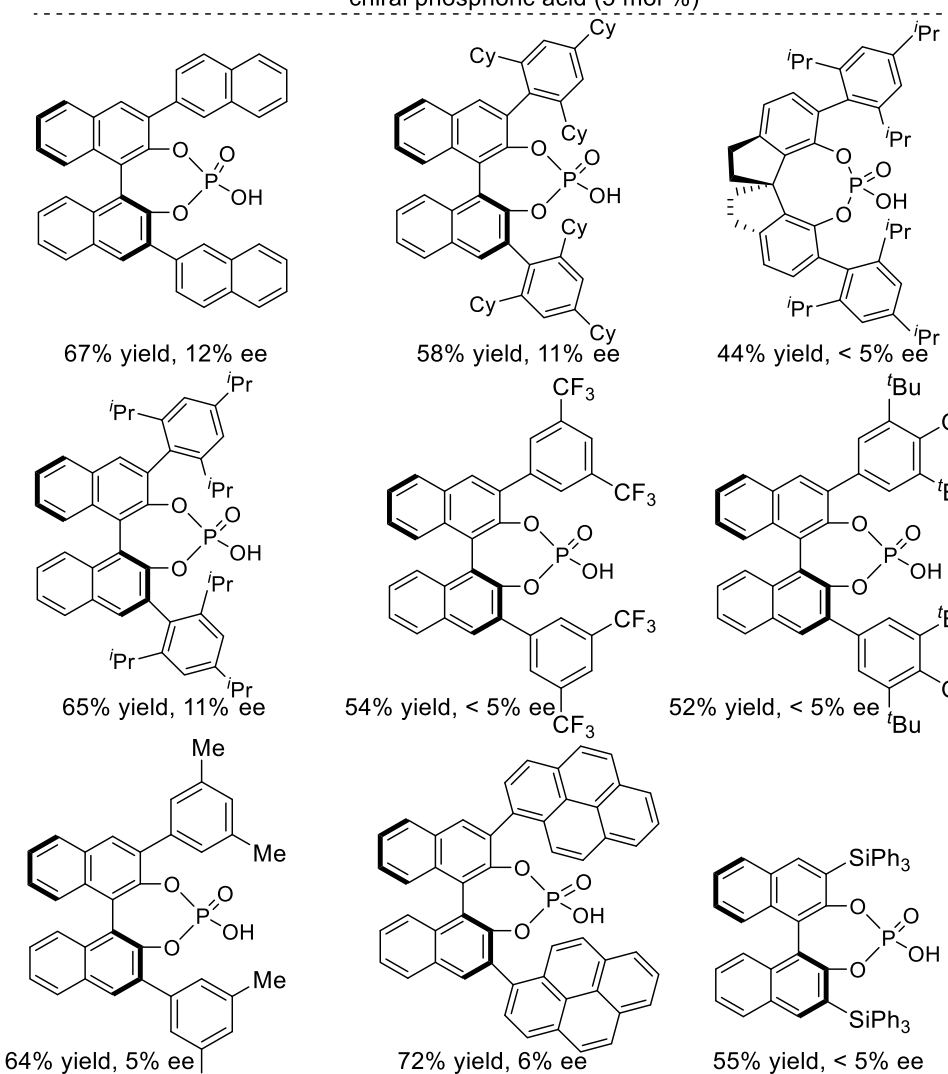

$54 \%$ yield, $<5 \%$ ee $\mathrm{CF}_{3}$
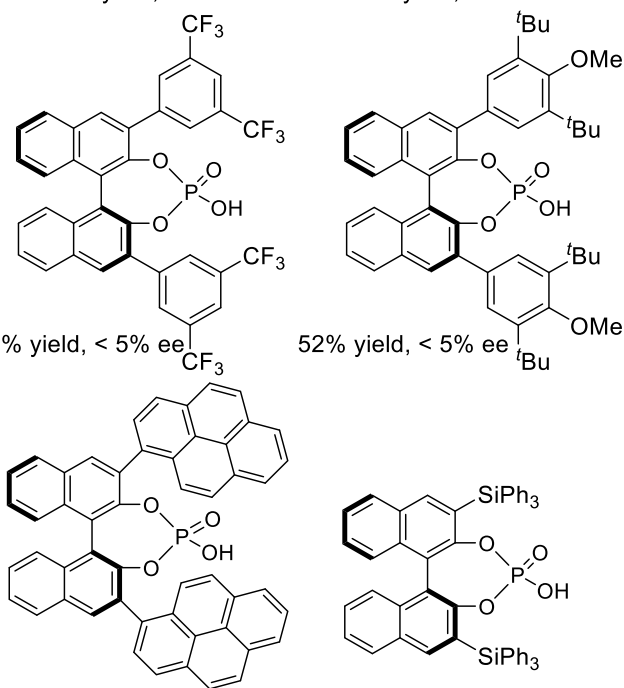

$72 \%$ yield, $6 \%$ ee

$52 \%$ yield, $<5 \%$ ee ${ }_{t_{\mathrm{Bu}}}$

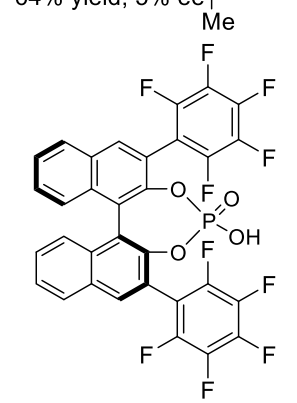

$48 \%$ yield, $<5 \%$ ee

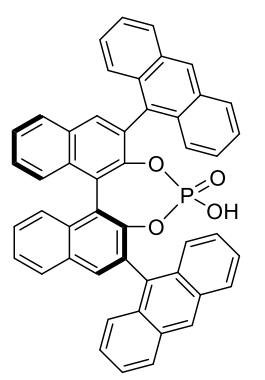

$67 \%$ yield, $<5 \%$ ee
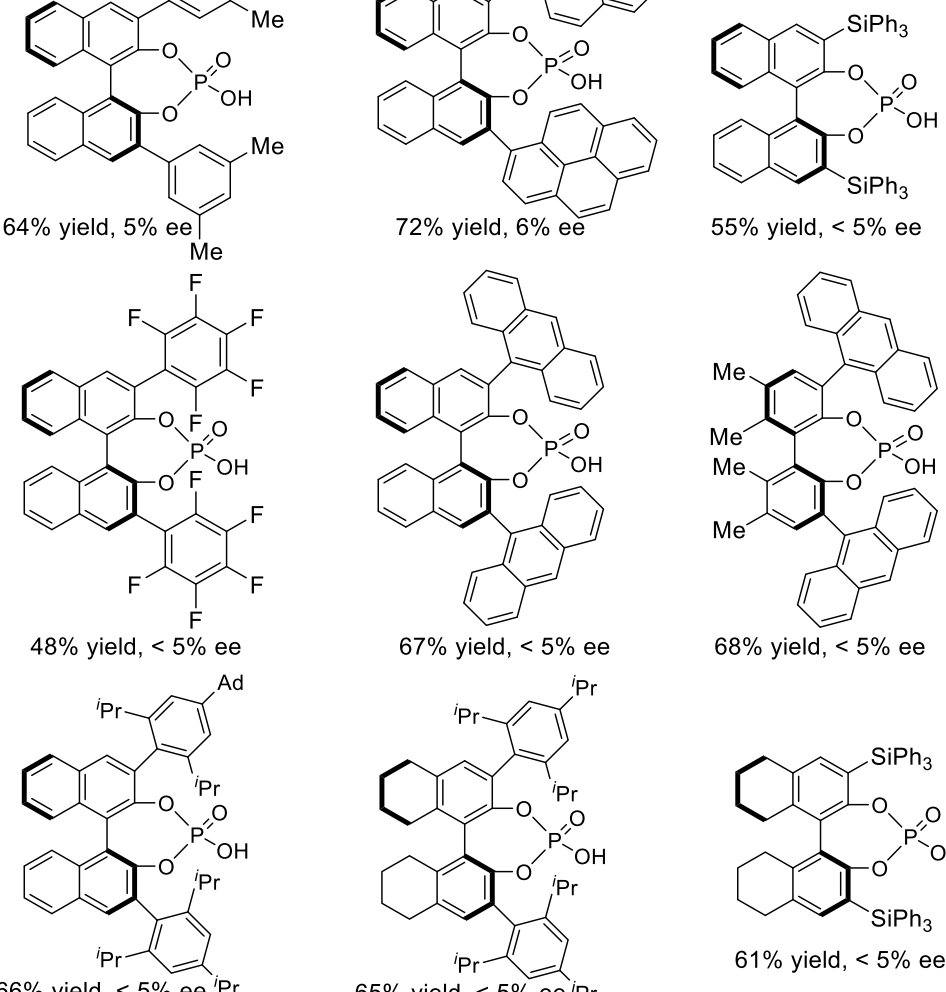

$55 \%$ yield, $<5 \%$ ee

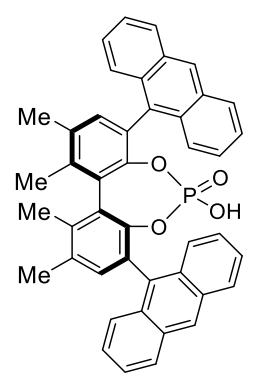

$68 \%$ yield, $<5 \%$ ee

$65 \%$ yield, $<5 \%$ ee ${ }^{i} \mathrm{Pr}$

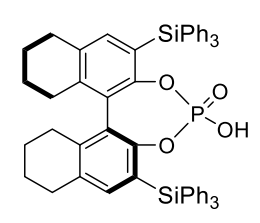

$61 \%$ yield, $<5 \%$ ee

General procedure for asymmetric version for 3a: To a dry Schlenk tube was added 1a (19.5 mg, $0.1 \mathrm{mmol})$, SPhosAuCl (3.2 mg, $0.005 \mathrm{mmol}), \mathrm{AgPF}_{6}(1.3 \mathrm{mg}, 0.005 \mathrm{mmol})$, chiral phosphoric acid $(0.005 \mathrm{mmol})$ and DCE $(1 \mathrm{~mL})$. The mixture was stirred at $\mathrm{rt}$ for $0.5 \mathrm{~h}$ under an argon atmosphere, then $2 \mathrm{a}(40.4 \mathrm{mg}, 0.2 \mathrm{mmol})$ in $1 \mathrm{~mL}$ of DCE was added via a syringe pump over $2 \mathrm{~h}$. The reaction mixture was concentrated under vacuum, the residue was purified by column chromatography (silica gel, petroleum ether/ethyl acetate $=20: 1$ to 10:1) to give 3a. The yields and ee values of different chiral phosphoric acids were listed in the above Scheme. The ee value 
was determined by HPLC: Daicel Chiralpak IC column, $n$-hexane/i-PrOH $=80 / 20$, flow rate 1.0 $\mathrm{mL} / \mathrm{min}, \lambda=254 \mathrm{~nm}, \mathrm{t}_{\mathrm{R}}=6.98 \mathrm{~min}$ (major) and $8.27 \mathrm{~min}$ (minor).

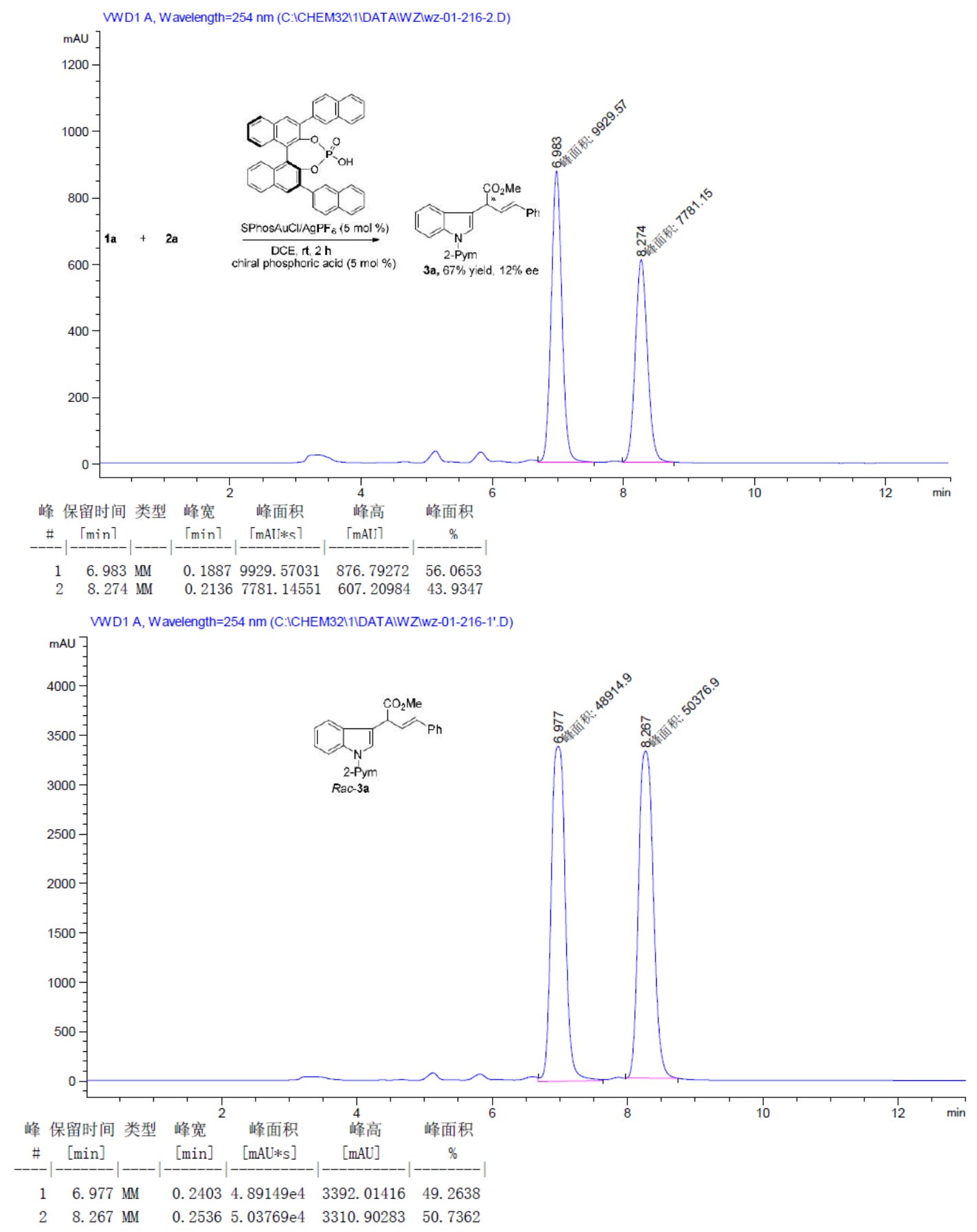

\section{$X$-ray structure of $3 a$ and $4 a$}

The crystal structures have been deposited at the Cambridge Crystallographic Data Centre (CCDC 1954345, 3a and CCDC 1954346, 4a). The data can be obtained free of charge via the internet at https: //www.ccdc.cam.ac.uk/structures/. 


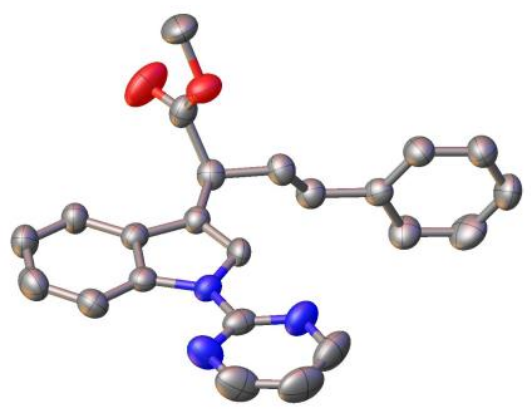

X-ray structure of $\mathbf{3 a}$

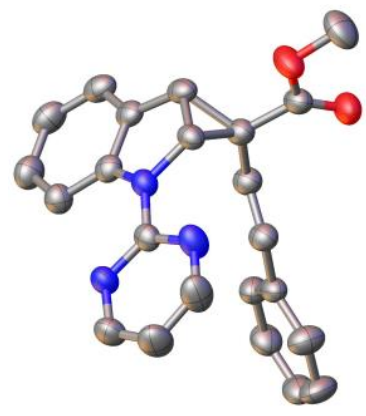

X-ray structure of $\mathbf{4 a}$

\section{References}

(1) (a) Ghorai, J.; Anbarasan, P. J. Org. Chem. 2015, 80, 3455. (b) Shi, J.-J.; Zhou, B.; Yang, Y.-X.; Li, Y.-C. Org. Biomol. Chem. 2012, 10, 8953. (c) Pan, C.-D.; Jin, H.-M.; Xu, P.; Liu, X.; Cheng, Y.-X.; Zhu, C.-J, J. Org. Chem. 2013, 78, 9494.

(2) (a) Davies, H. M. L.; Yang, J.; Manning, J. R. Tetrahedron: Asymmetry 2006, 17, 665. (b) Manning, J. R.; Davies, H. M. L.Org. Synth. 2007, 84, 334. 


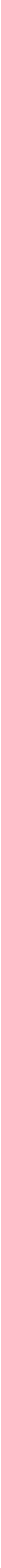




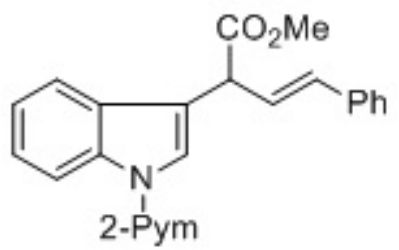

$100 \mathrm{MHz}, \mathrm{CDCl}_{3}$

3a

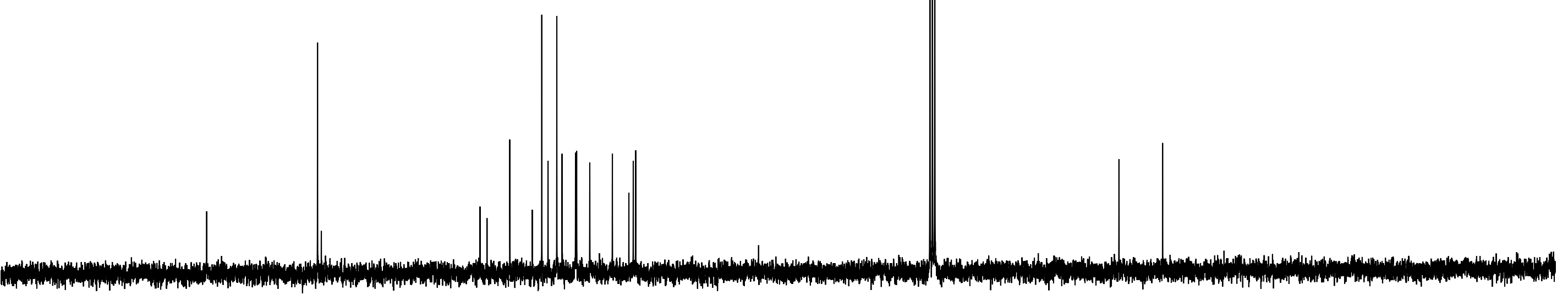$$
0
$$

190

80

70

70 


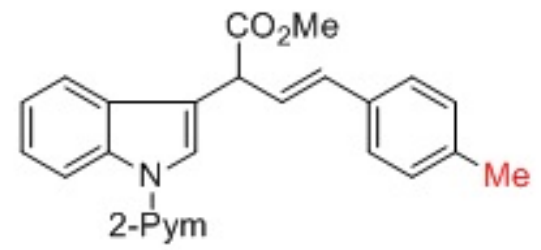

$75 \mathrm{MHz}, \mathrm{CDCl}_{3}$

3b

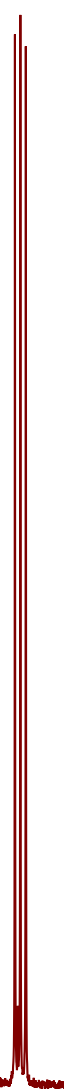

$-6000$

$\begin{array}{llllll}180 & 180 & 170 & 160 & 150 & 140\end{array}$

$130 \quad 120$

$\begin{array}{lcc}110 & 100 & 90 \\ & f 1(\mathrm{ppm}) & \end{array}$




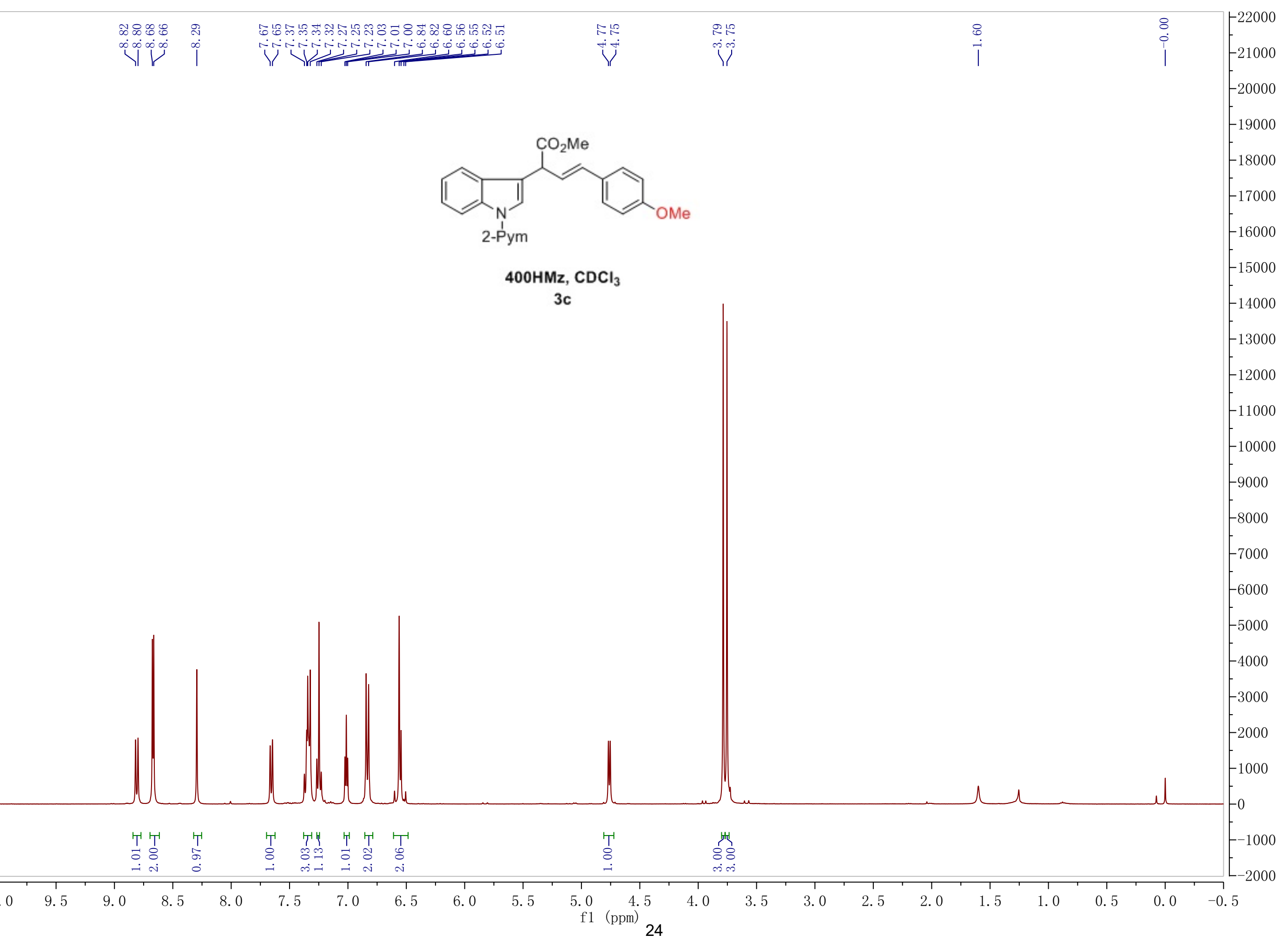




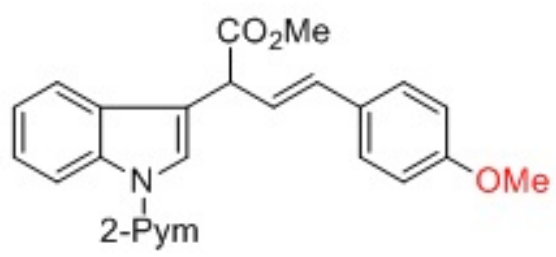

$75 \mathrm{MHz}, \mathrm{CDCl}_{3}$

$3 \mathrm{c}$

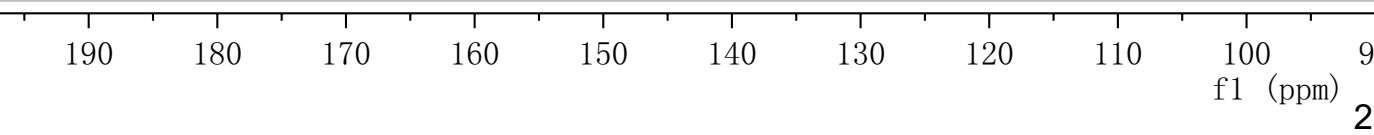




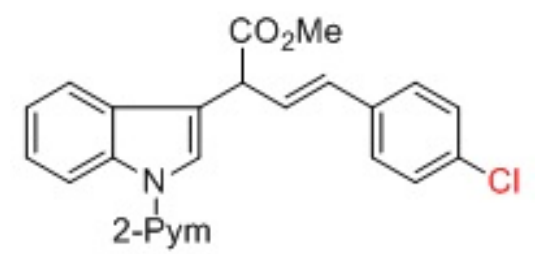

$300 \mathrm{MHz}, \mathrm{CDCl}_{3}$

3d

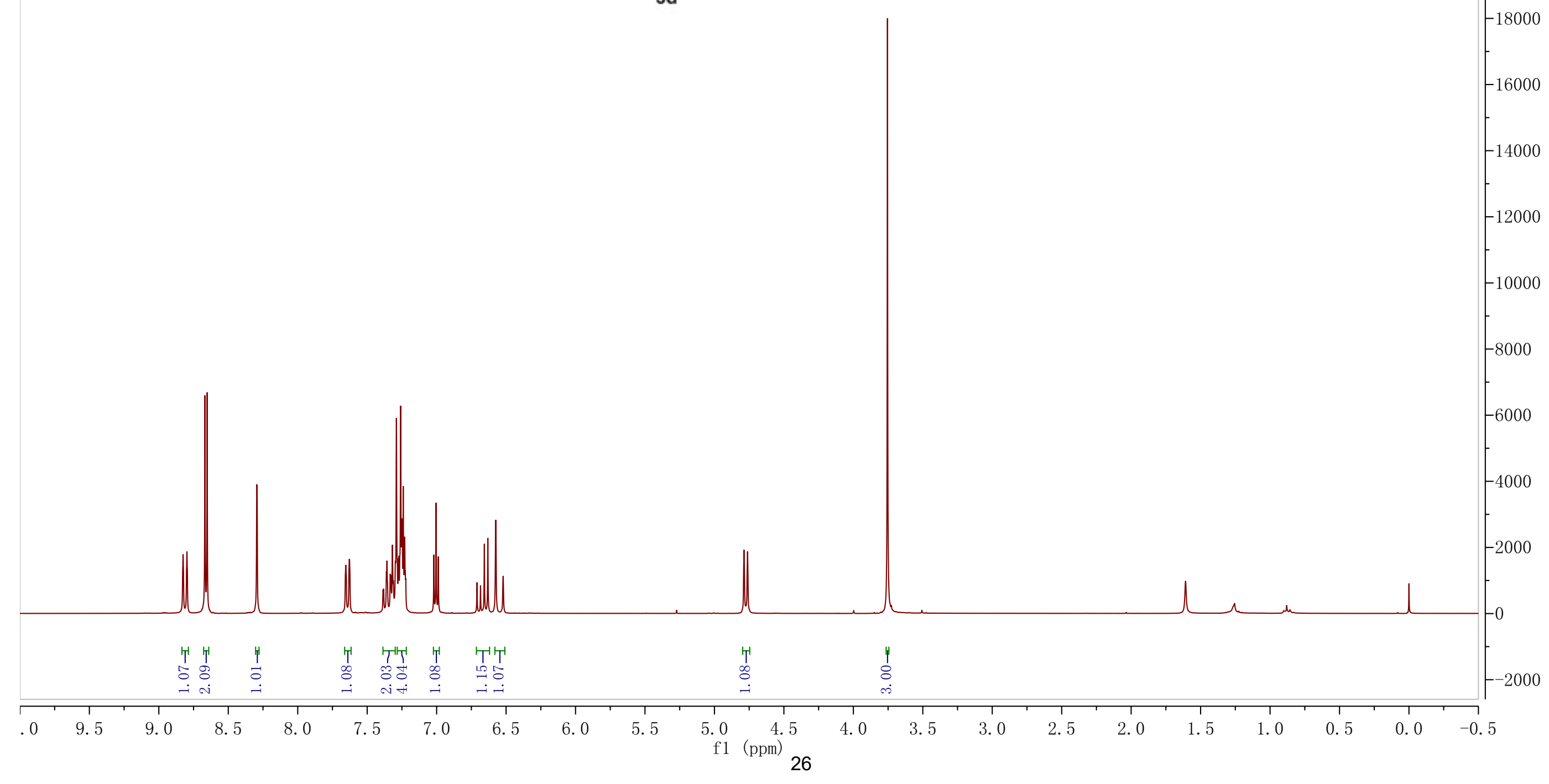




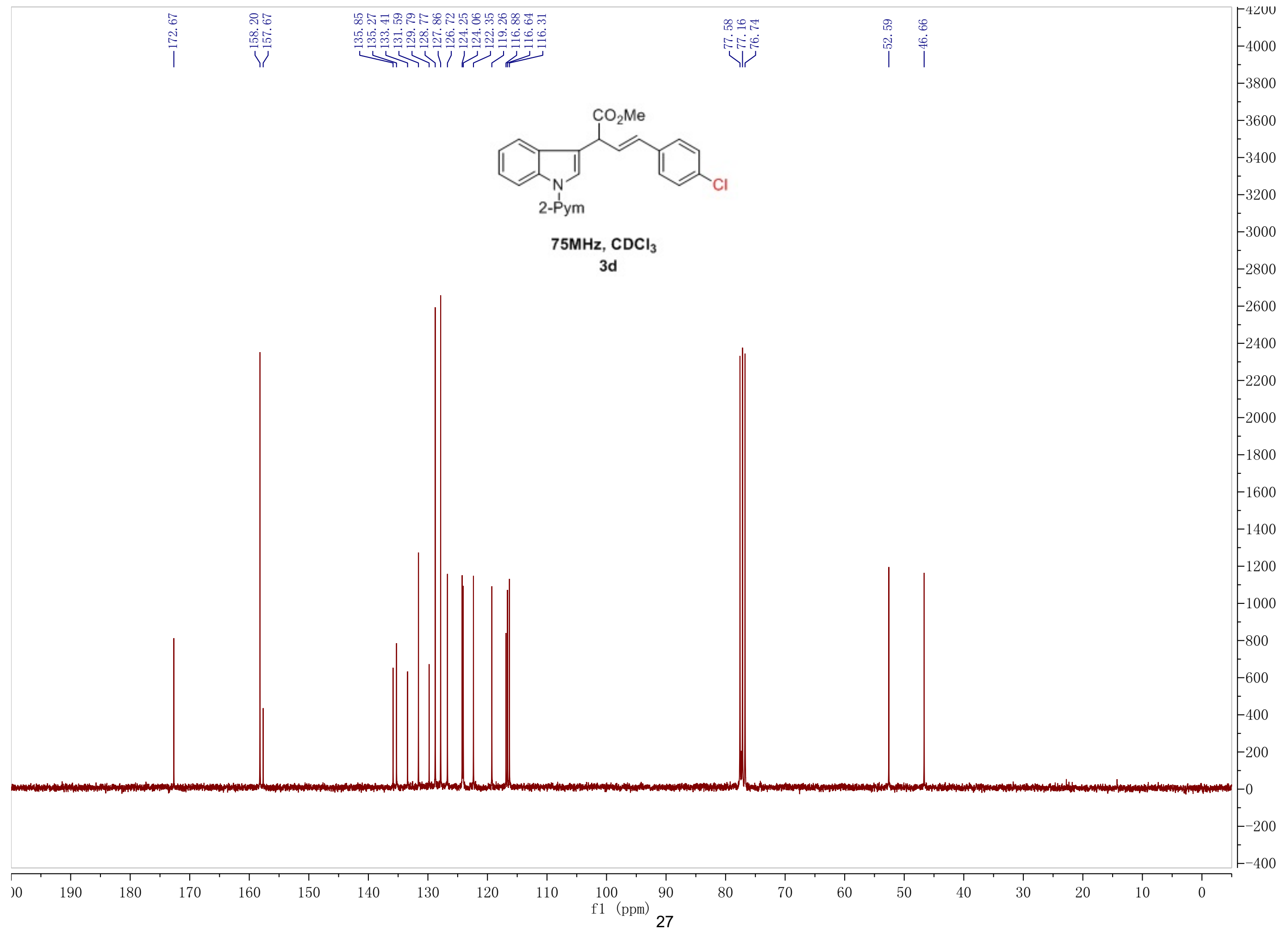




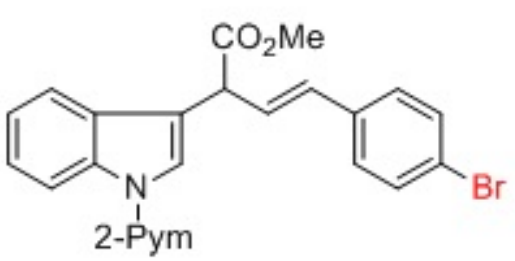

$400 \mathrm{MHz}_{3} \mathrm{CDCl}_{3}$

$3 e$

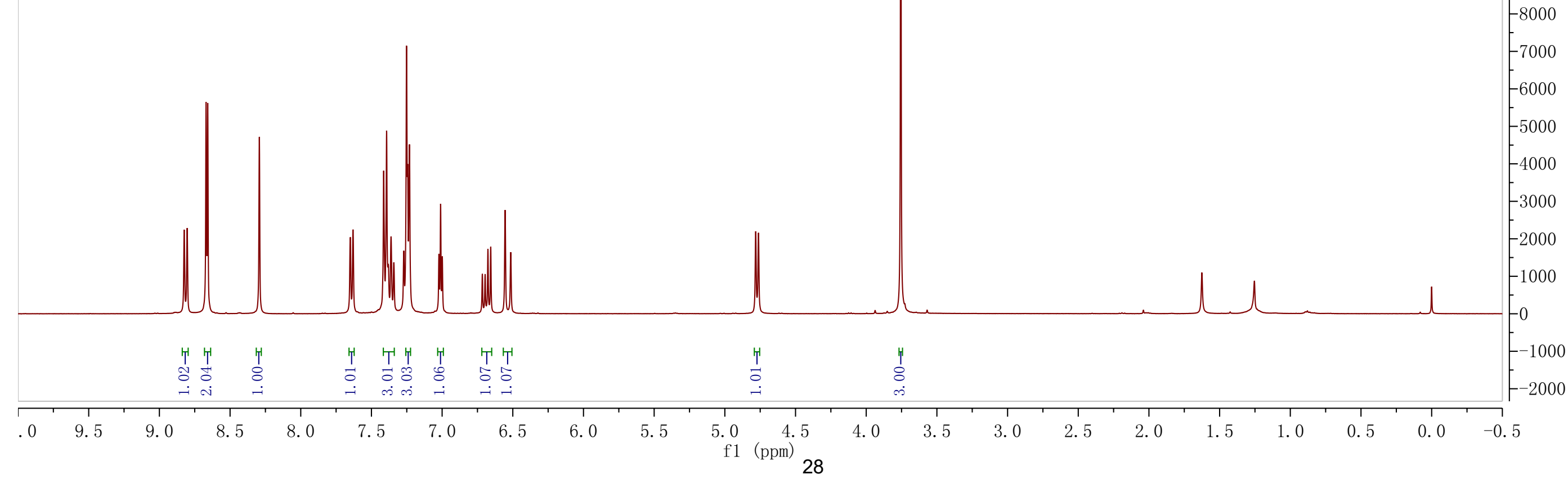




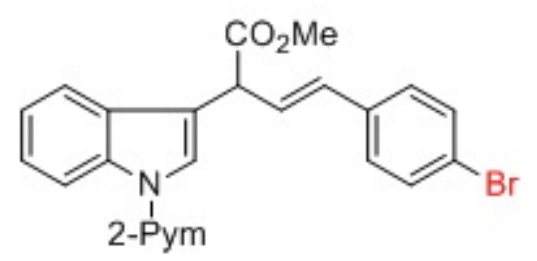

$75 \mathrm{MHz}, \mathrm{CDCl}_{3}$

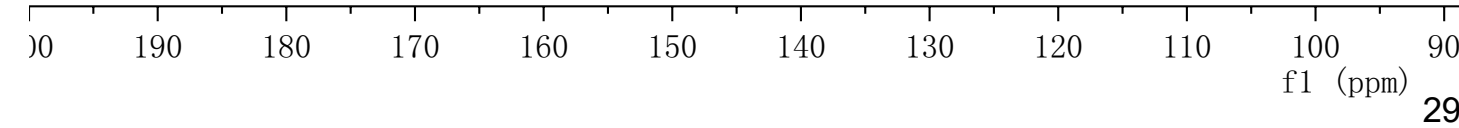




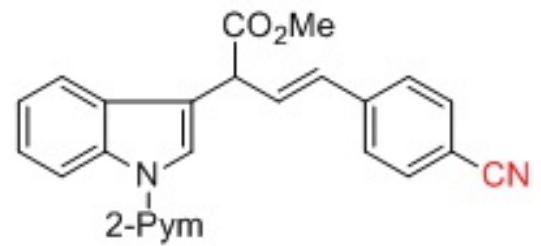

$75 \mathrm{MHz}, \mathrm{CDCl}_{3}$

$3 f$

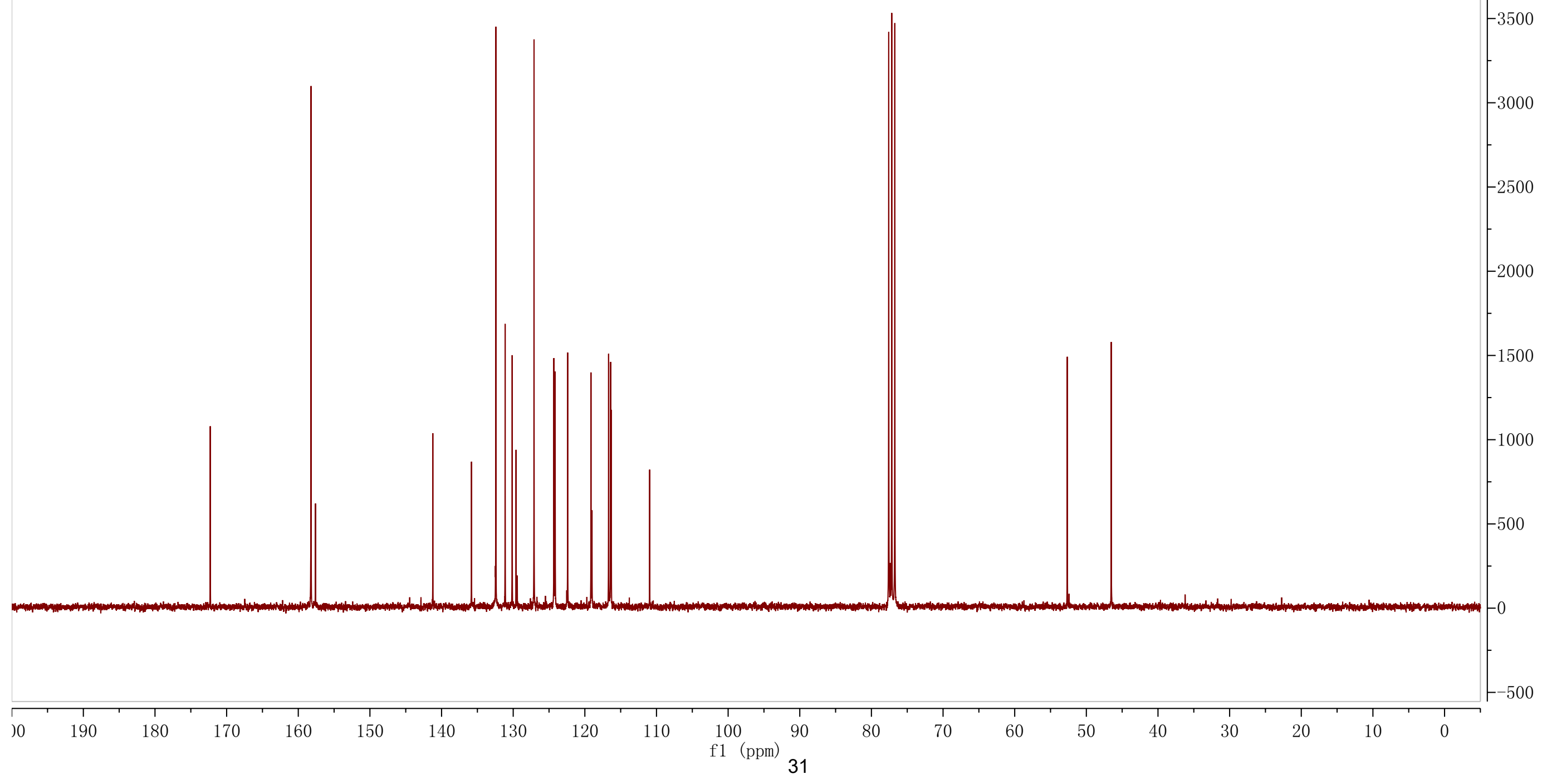




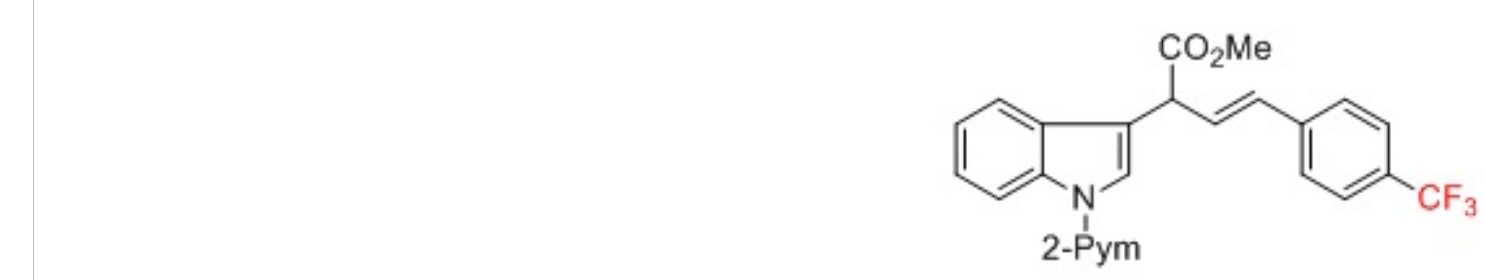

$300 \mathrm{MHz}, \mathrm{CDCl}_{3}$

$3 \mathrm{~g}$

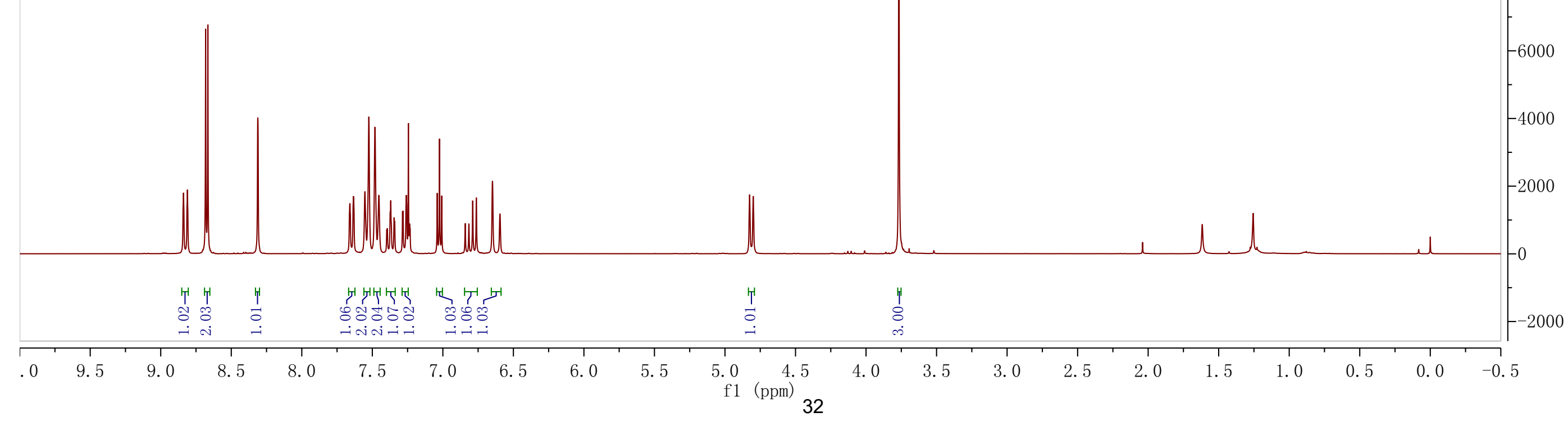




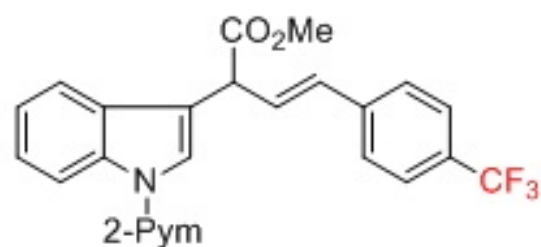

$75 \mathrm{MHz}, \mathrm{CDCl}_{3}$

$3 \mathrm{~g}$

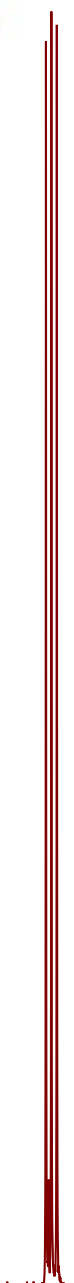




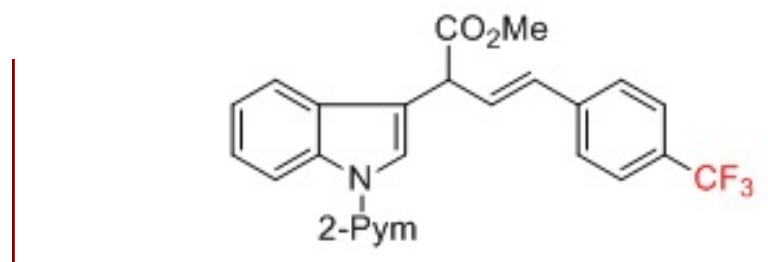

$282 \mathrm{MHz} \mathrm{CDCl}_{3}$

$3 \mathrm{~g}$ 


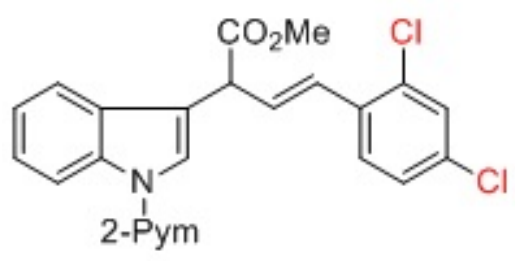

\section{$75 \mathrm{MHz} \mathrm{CDCl}_{3}$}

$3 \mathrm{~h}$

$\begin{array}{lllllllll}180 & 170 & 160 & 150 & 140 & 130 & 120 & 110 & \begin{array}{c}100 \\ \mathrm{f} 1(\mathrm{ppm})\end{array}\end{array}$




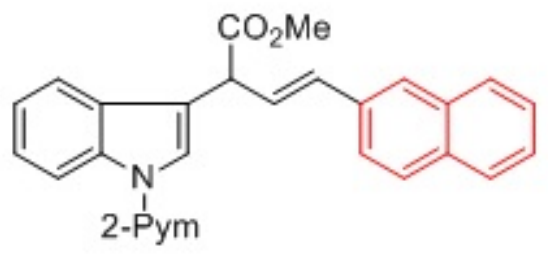

$300 \mathrm{MHz}, \mathrm{CDCl}_{3}$

$3 i$

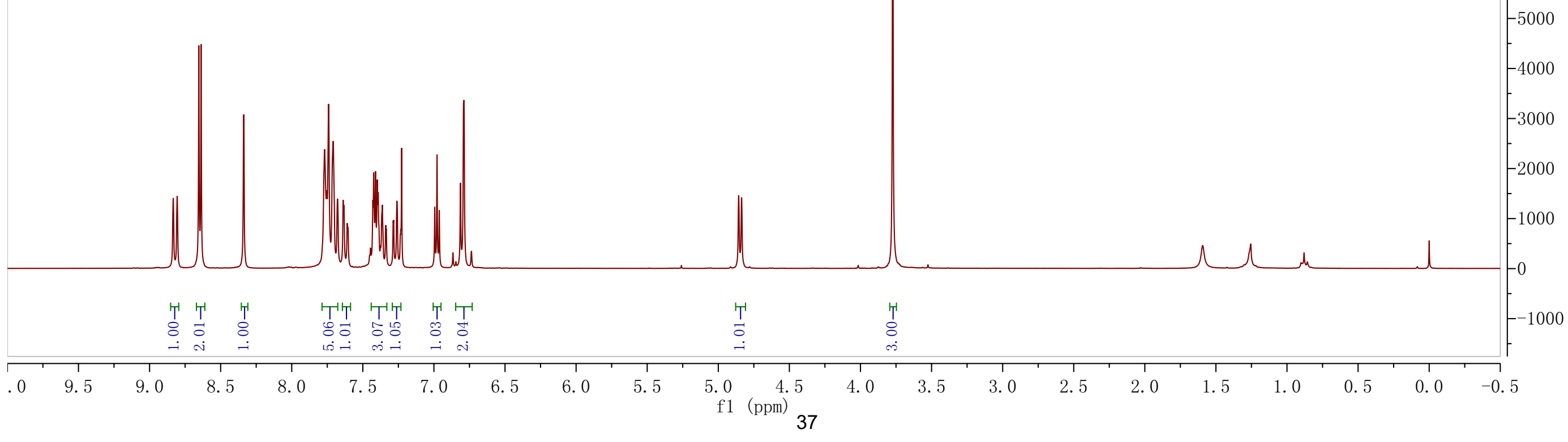




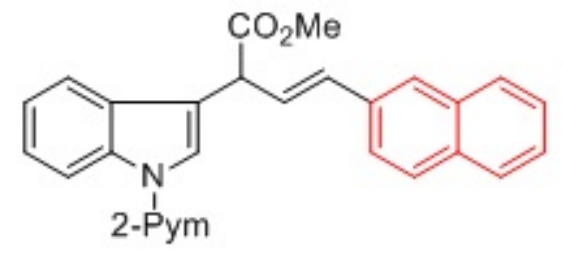

$75 \mathrm{MHz}, \mathrm{CDCl}_{3}$

$3 i$ 


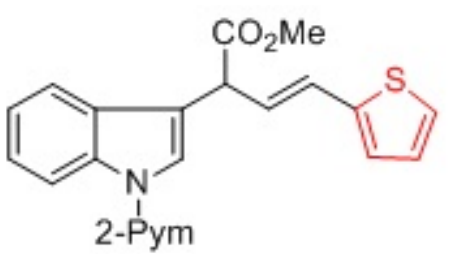

\section{$300 \mathrm{MHz}, \mathrm{CDCl} 3$}

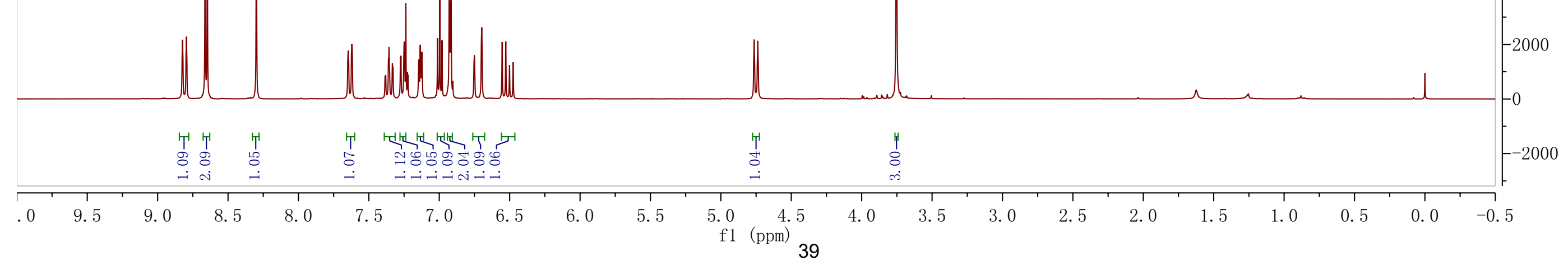




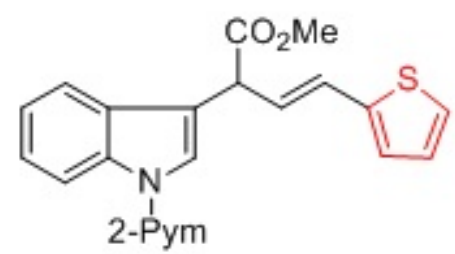

$300 \mathrm{MHz}, \mathrm{CDCl}_{3}$

$3 \mathrm{j}$

180




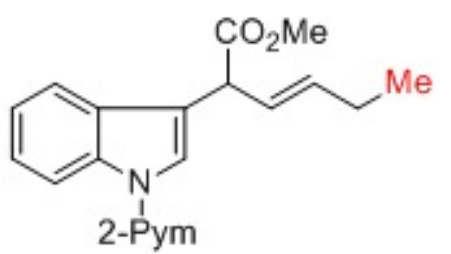

$400 \mathrm{MHz}_{3} \mathrm{CDCl}_{3}$
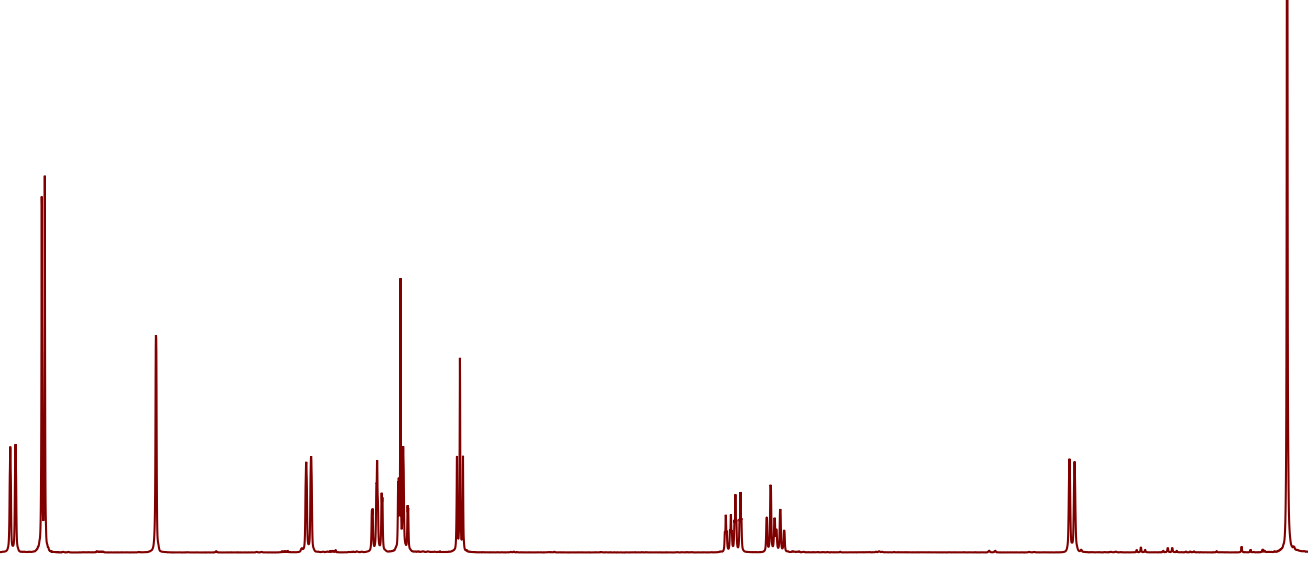

\section{dis}

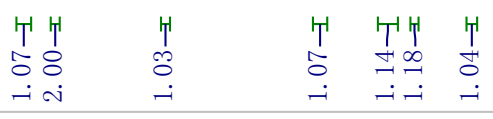

을

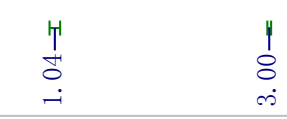




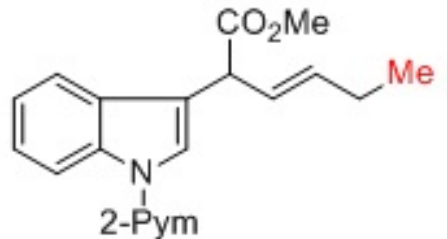

\section{$75 \mathrm{MHz}, \mathrm{CDCl}_{3}$}

$3 k$

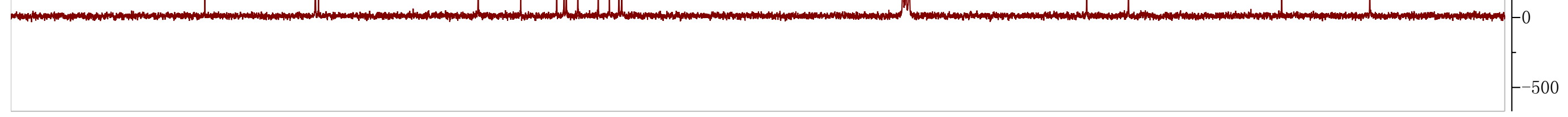

\begin{tabular}{|c|c|c|c|c|c|c|c|c|c|c|}
\hline$) 0$ & 190 & 180 & 170 & 160 & 150 & 140 & 130 & 120 & 110 & $\begin{array}{l}100 \\
\mathrm{f} 1 \quad(\mathrm{ppm})\end{array}$ \\
\hline
\end{tabular}




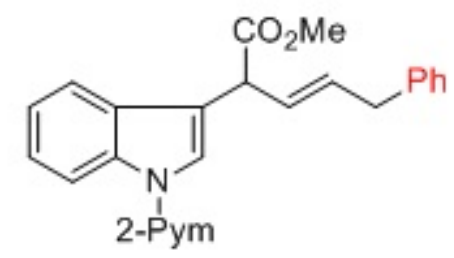

$300 \mathrm{MHz}, \mathrm{CDCl}_{3}$

3।
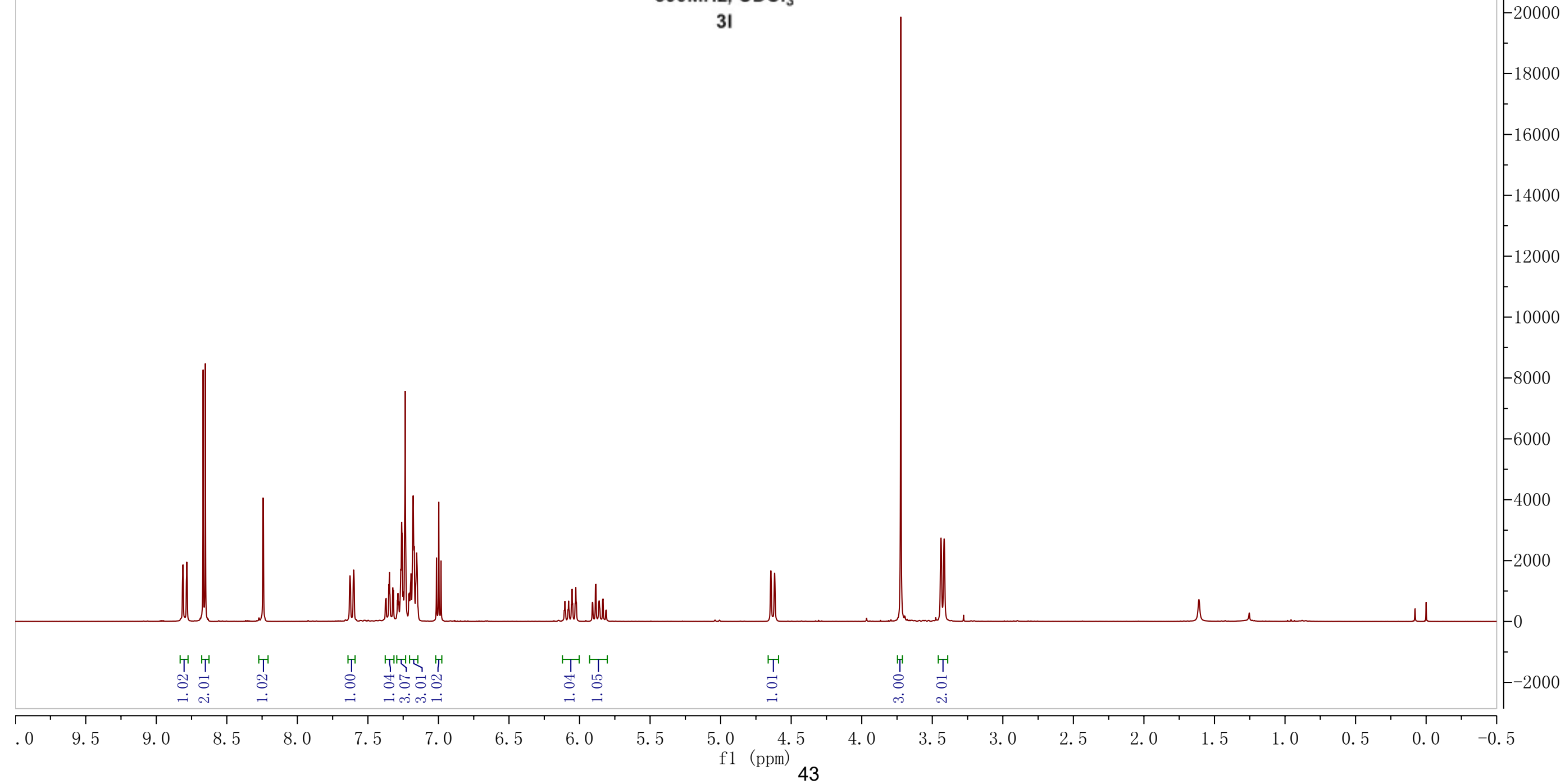


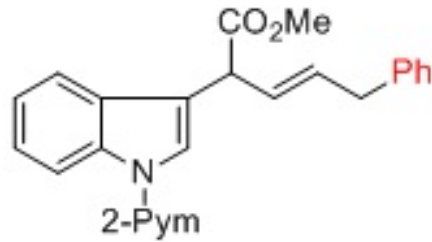

$75 \mathrm{MHz}, \mathrm{CDCl}_{3}$

31

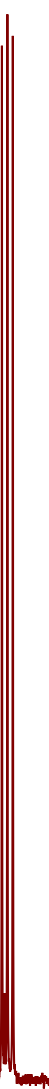


<smiles>CC(=O)C(C=Cc1ccccc1)c1cn(P)c2cccc(F)c12</smiles>

$300 \mathrm{MHz}, \mathrm{CDCl}_{3}$

$3 \mathrm{~m}$
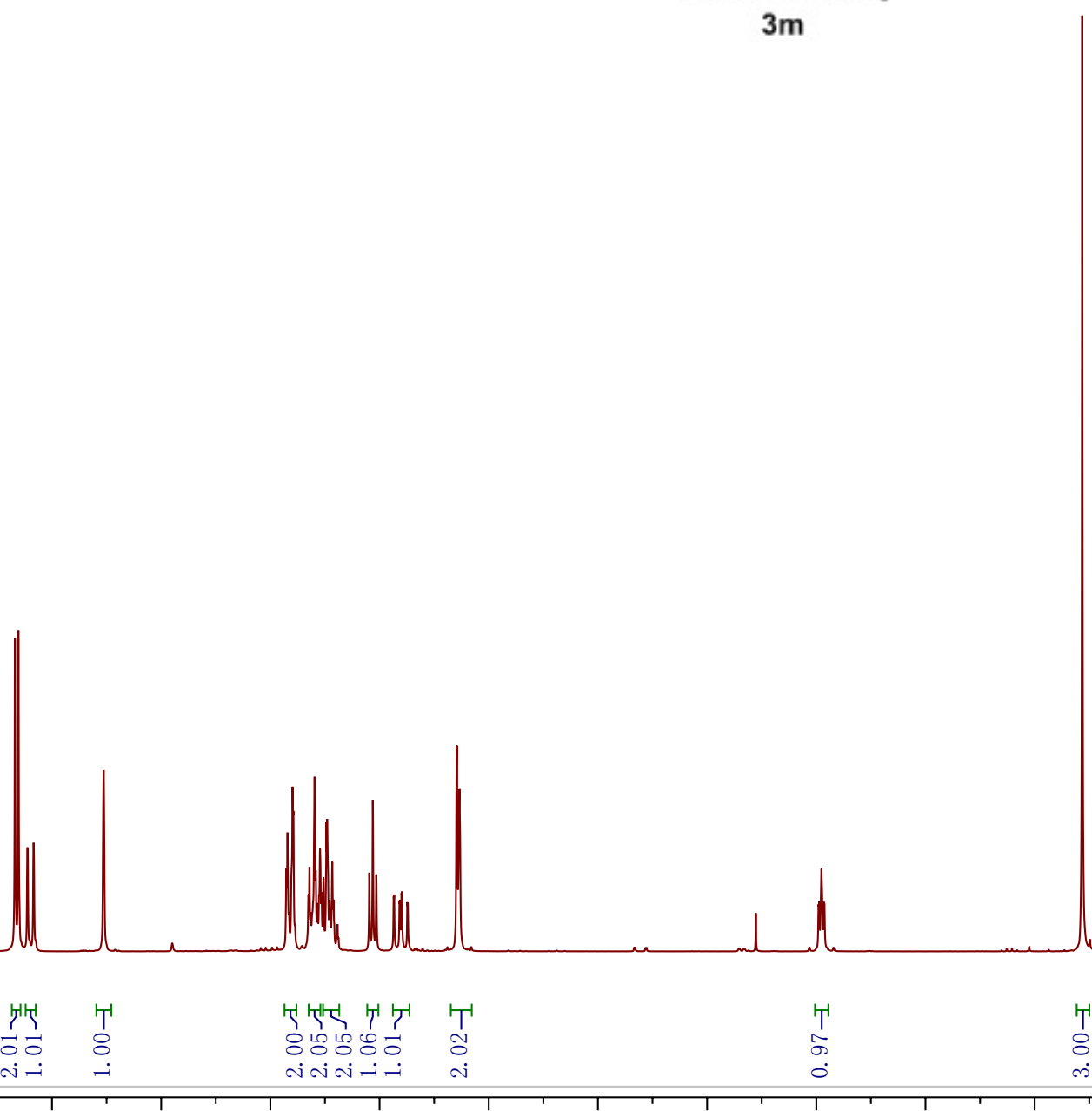


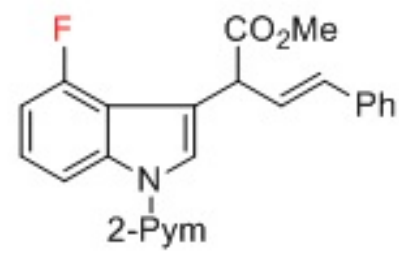

$300 \mathrm{MHz}, \mathrm{CDCl}_{3}$ 


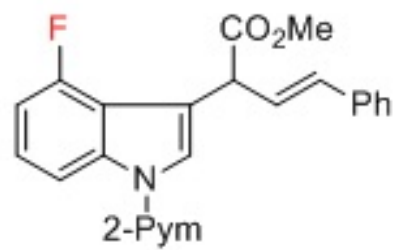

$282 \mathrm{MHz}, \mathrm{CDCl}_{3}$

$3 \mathrm{~m}$

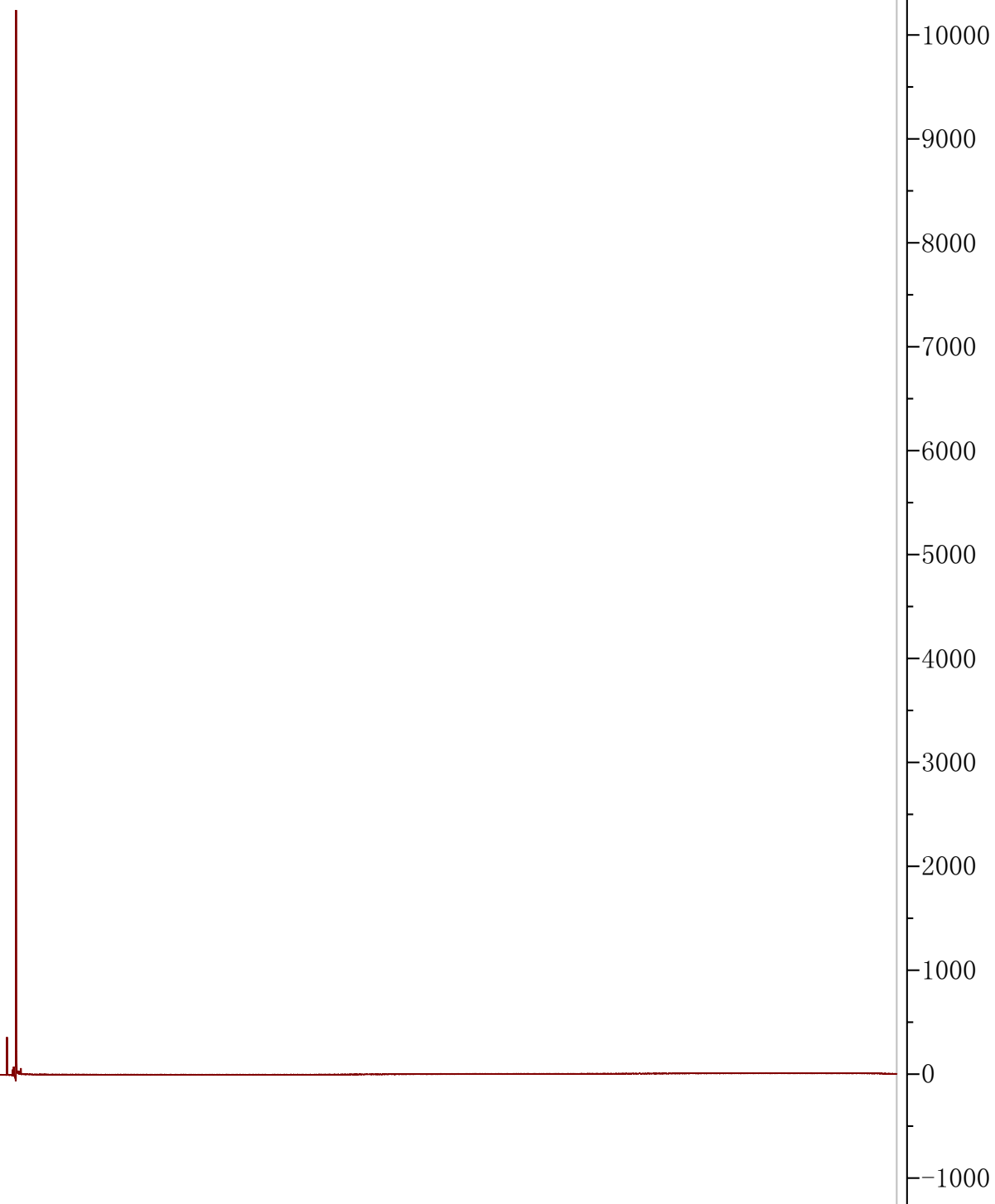




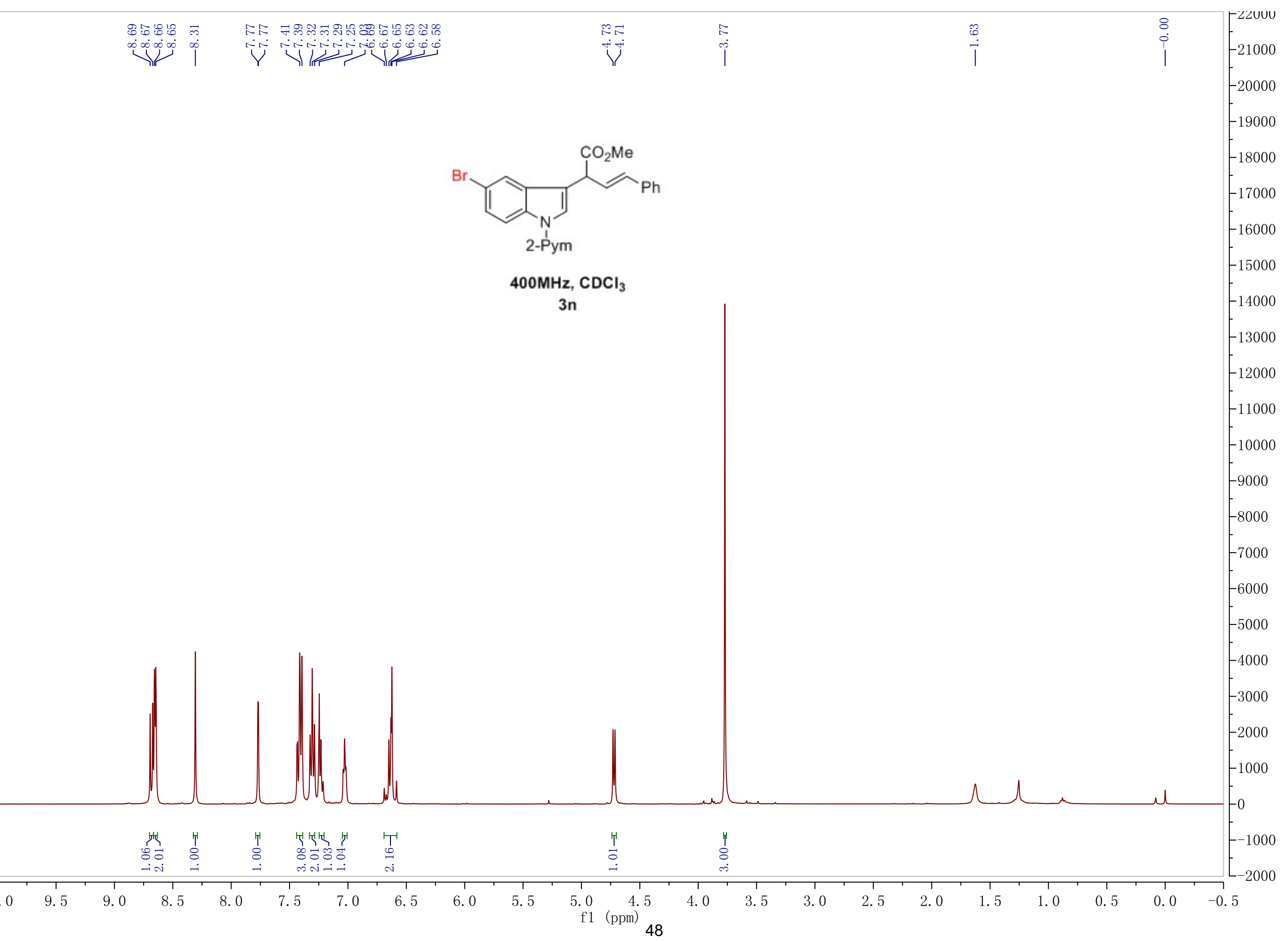




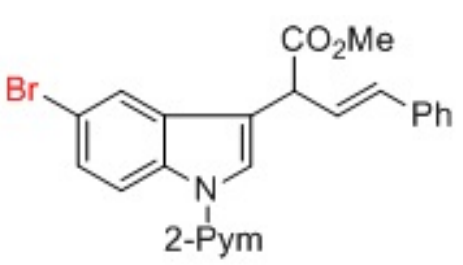

$100 \mathrm{MHz} \mathrm{CDCl}_{3}$ 


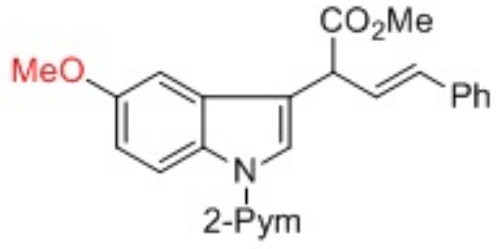

$75 \mathrm{MHz}, \mathrm{CDCl}_{3}$ 3o 


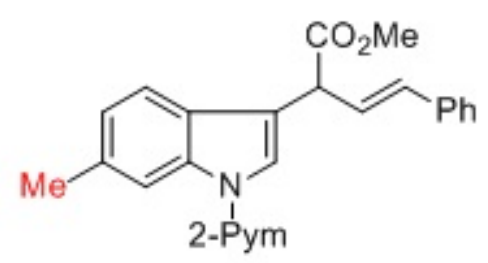

$400 \mathrm{MHz}_{3} \mathrm{CDCl}_{3}$

$3 p$

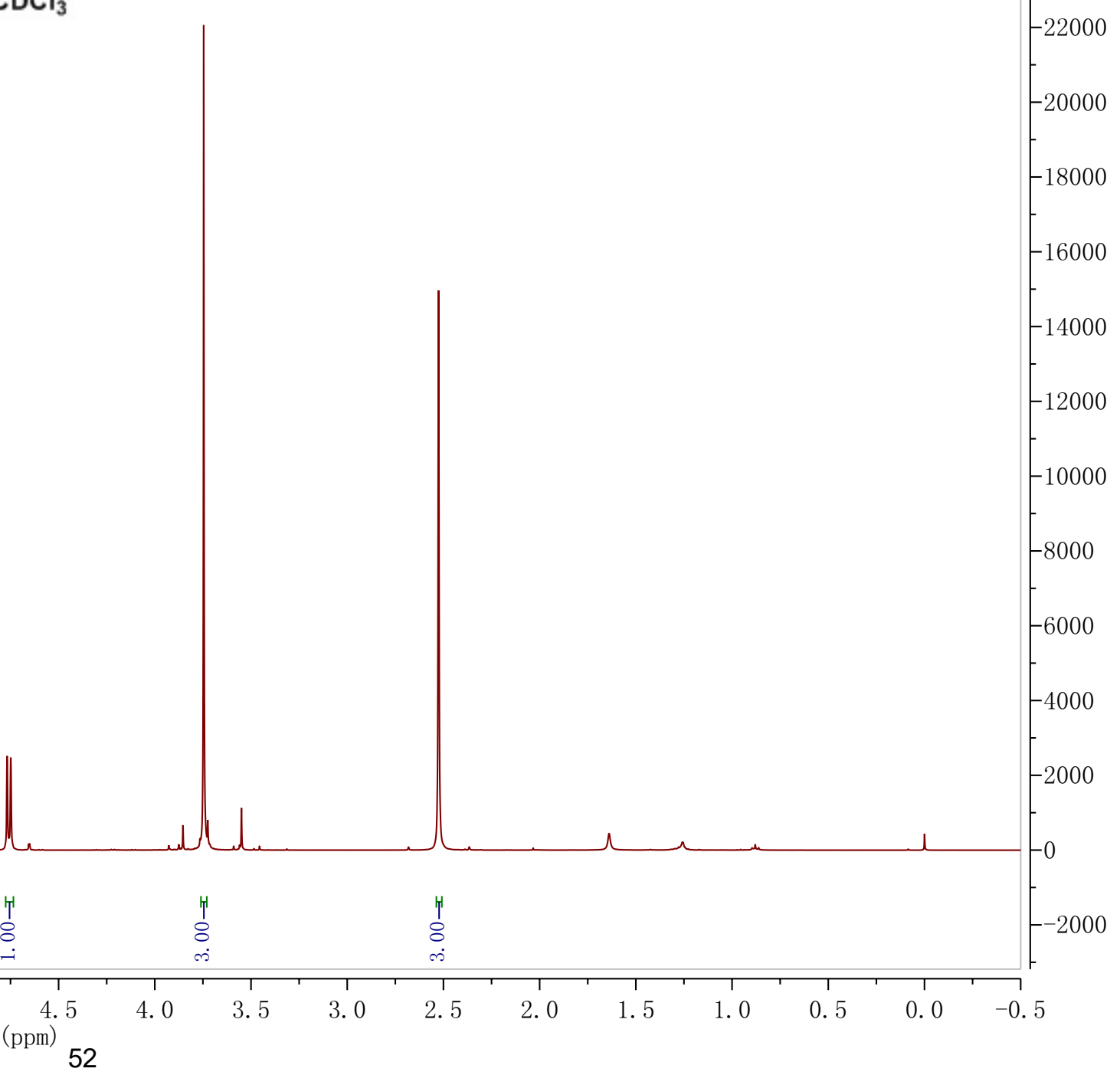




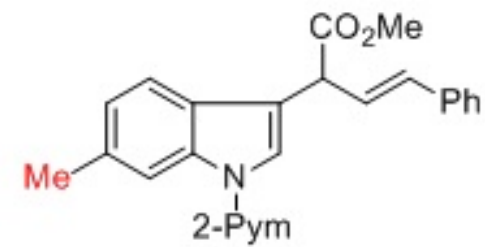

$75 \mathrm{MHz} \mathrm{CDCl}_{3}$

$3 p$ 


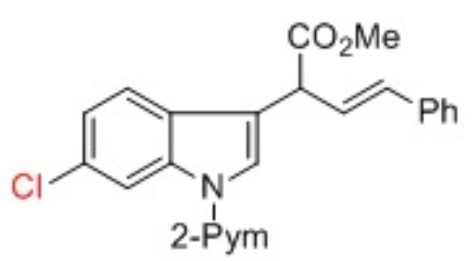

$400 \mathrm{MHz} \mathrm{CDCl}_{3}$

$3 q$ 
<smiles>CCCCN(C=CC(C=Cc1ccccc1)c1cn(P)c2cc(Cl)ccc12)CC</smiles>

$100 \mathrm{MHz}, \mathrm{CDCl}_{3}$

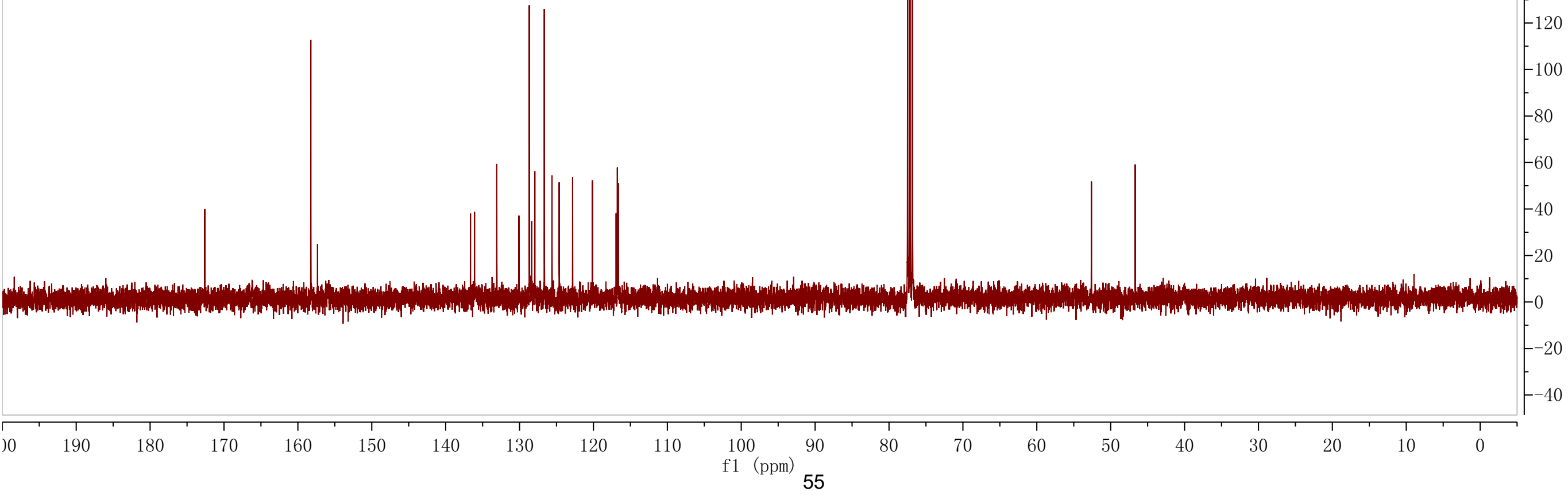




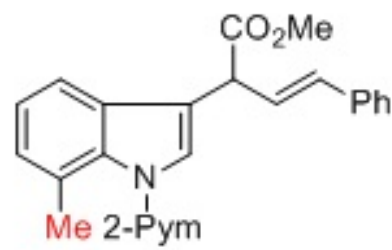

\section{$400 \mathrm{MHz}$, DMSO-d}

$$
3 r
$$

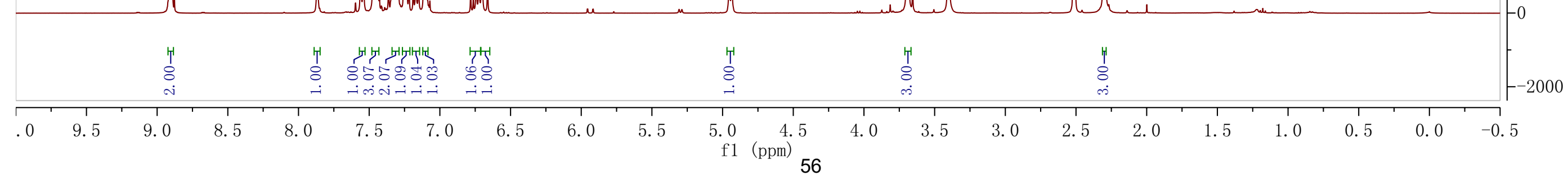




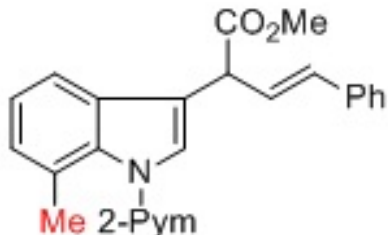

$100 \mathrm{MHz}, \mathrm{CDCl}_{3}$ 


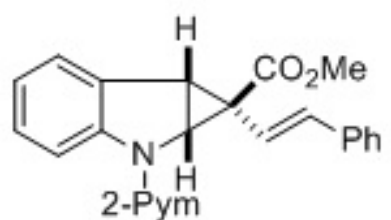

$400 \mathrm{MHz}, \mathrm{CDCl} 3$

$4 a$

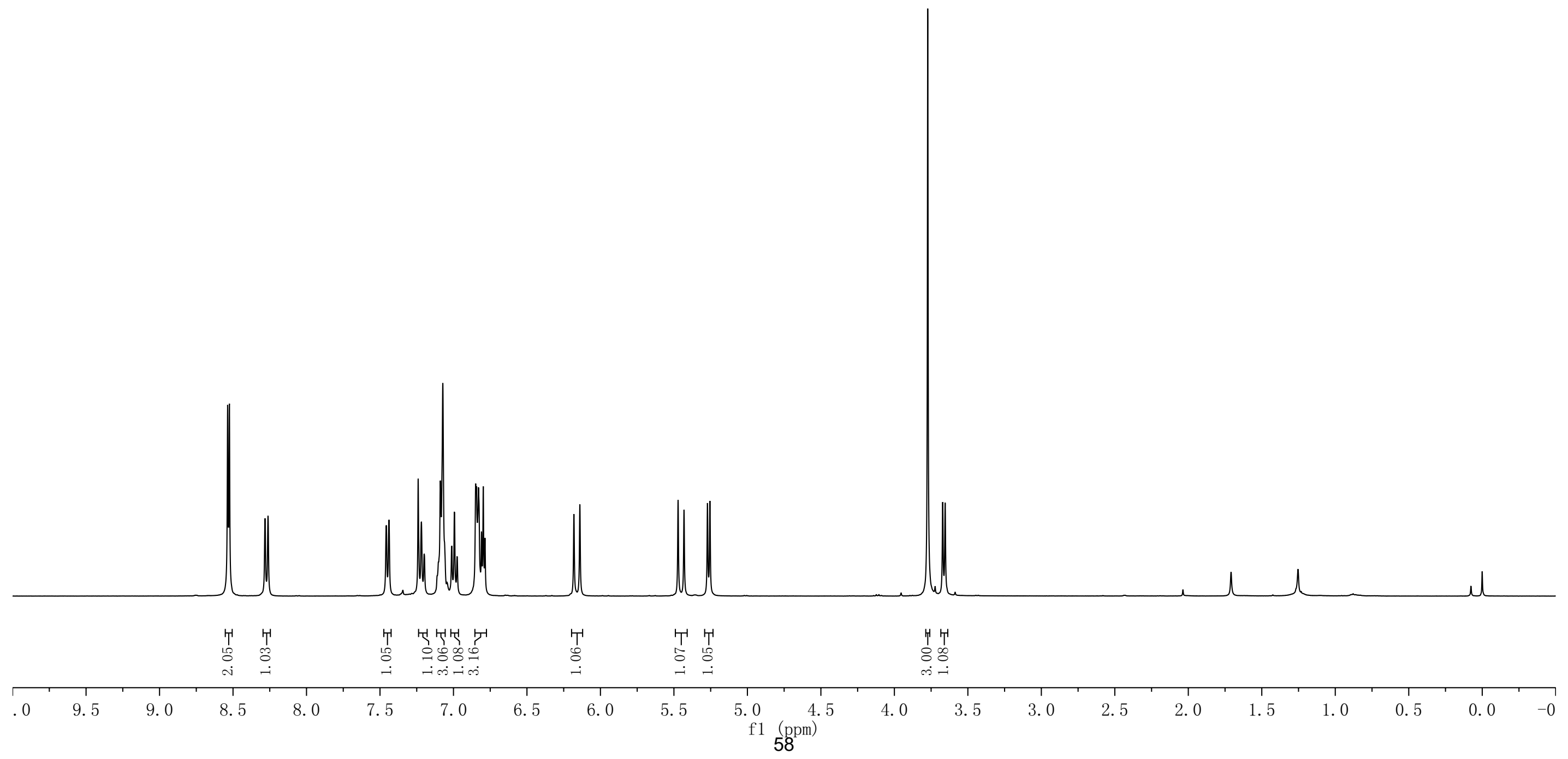




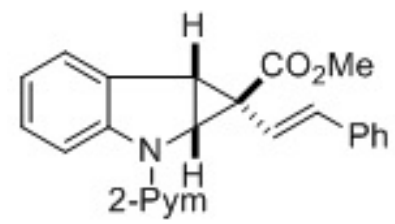

$75 \mathrm{MHz}, \mathrm{CDCl}^{3}$ 


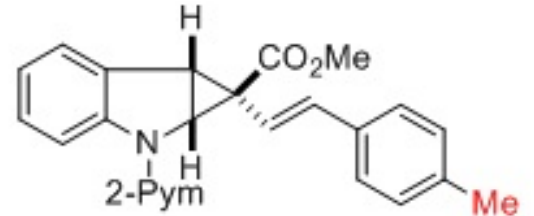

$300 \mathrm{MHz}, \mathrm{CDCl}_{3}$

$4 b$

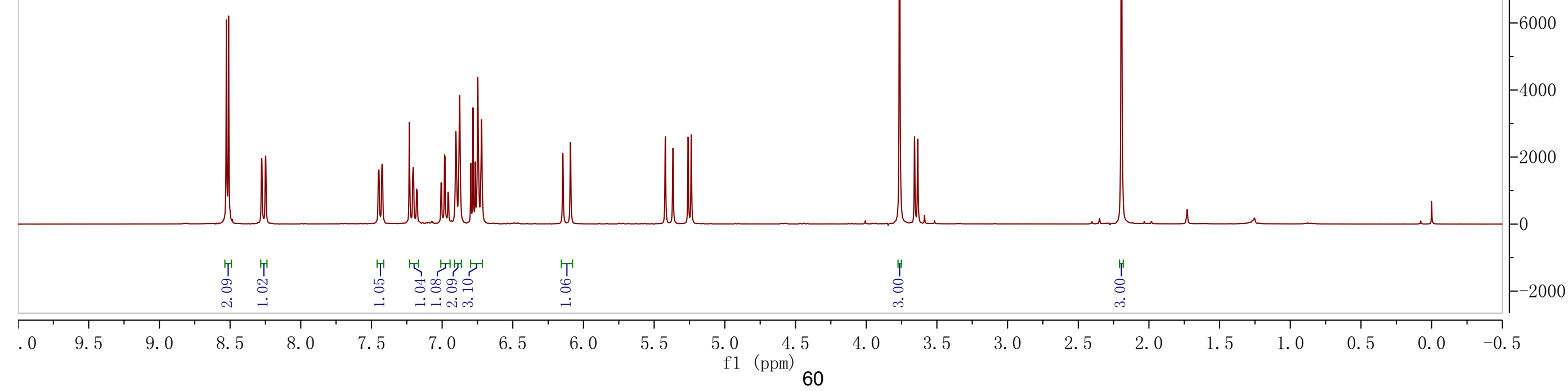




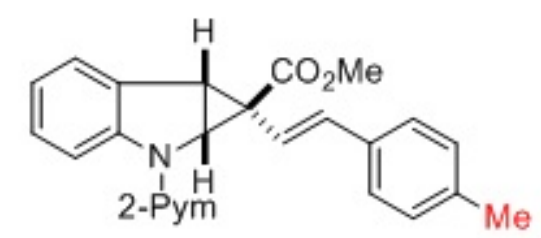

$75 \mathrm{MHz}, \mathrm{CDCl}_{3}$

4b

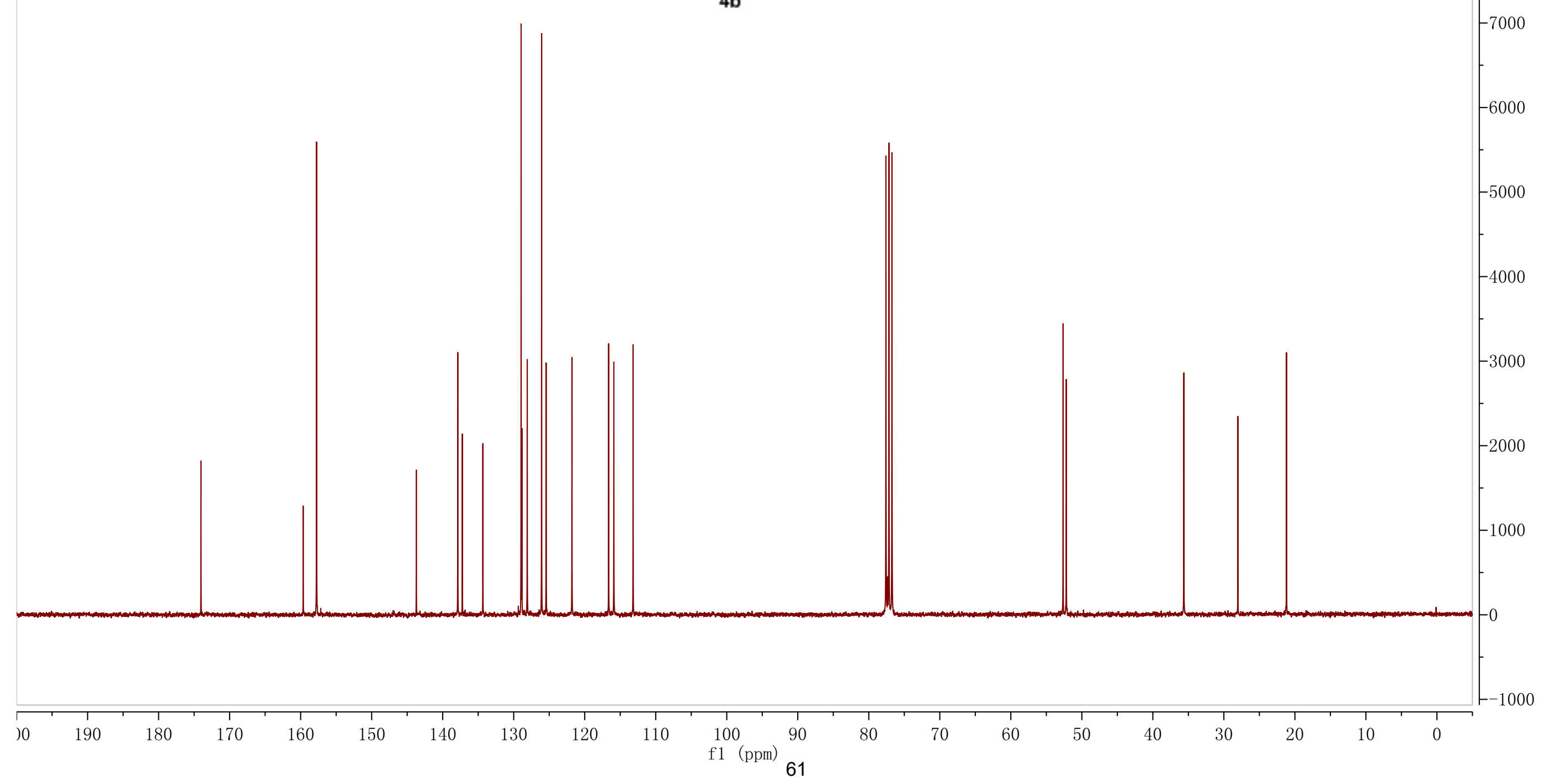




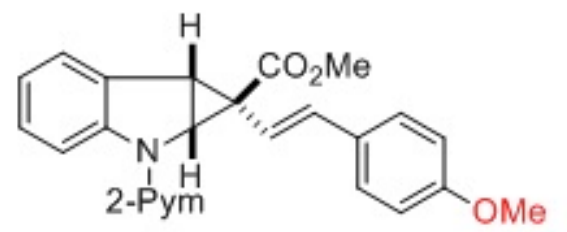

$400 \mathrm{MHz}, \mathrm{CDCl}_{3}$

$4 \mathrm{c}$

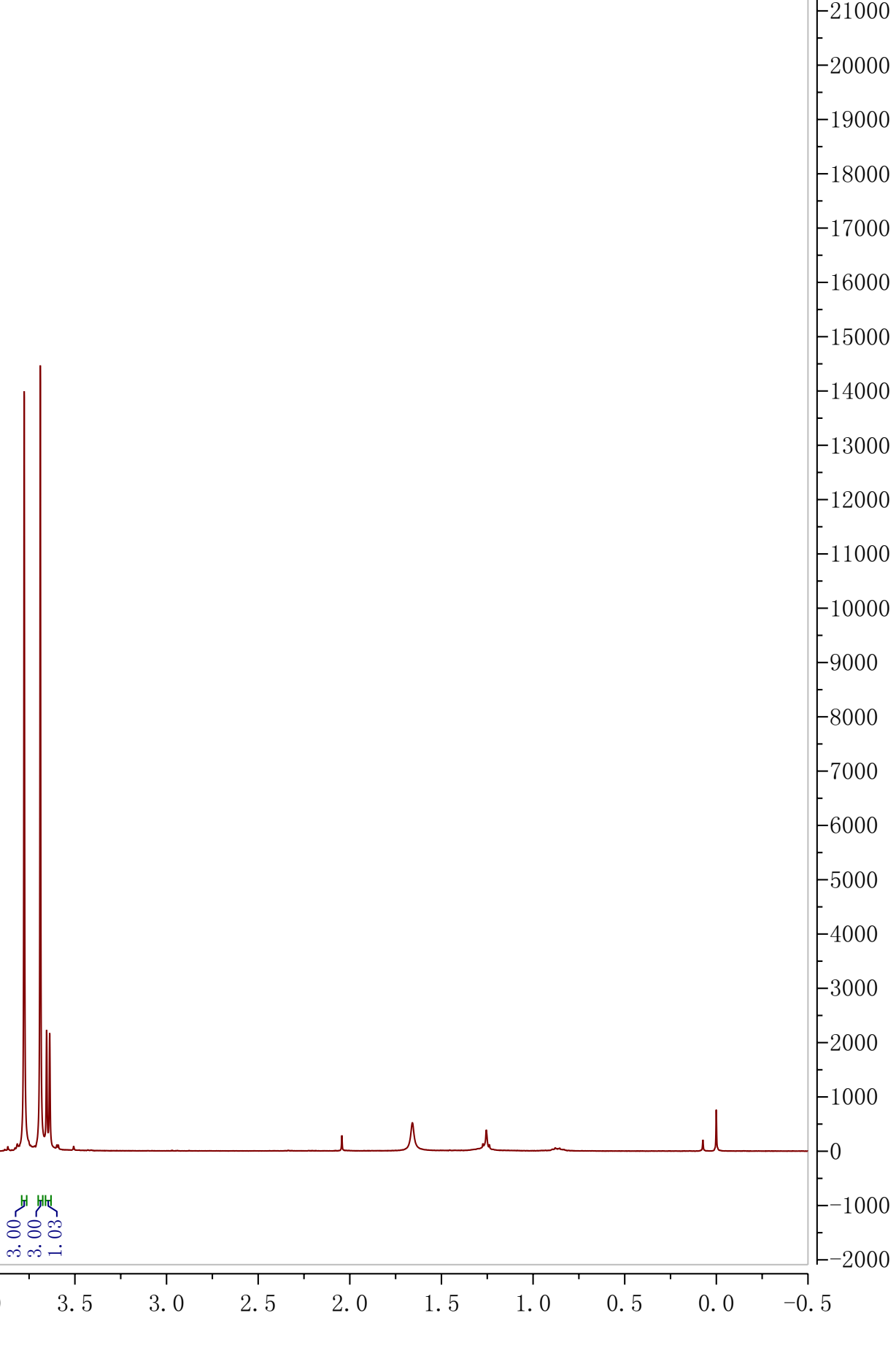




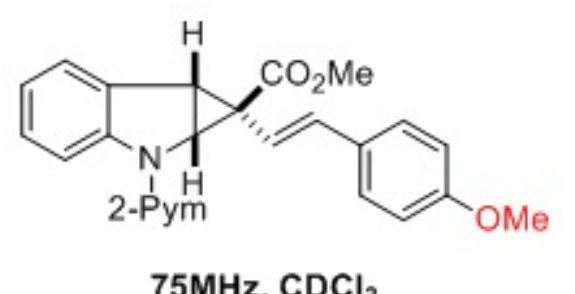

$4 c$

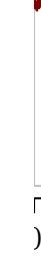




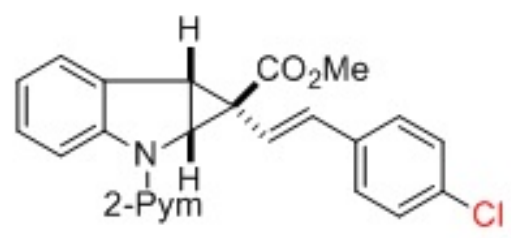

$400 \mathrm{MHz}, \mathrm{CDCl}_{3}$

$4 d$

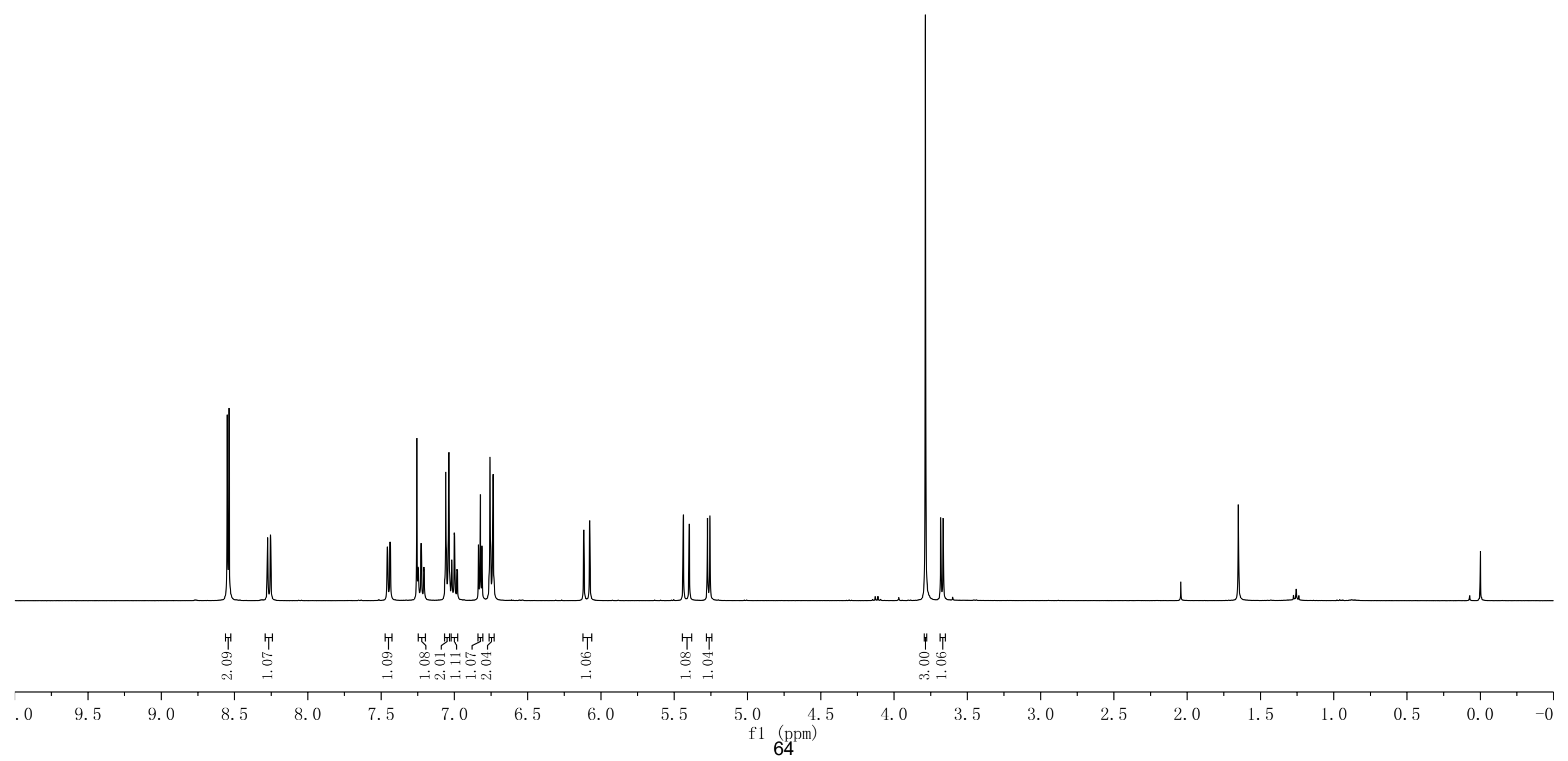




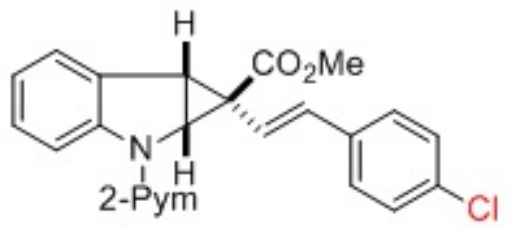

$75 \mathrm{MHz}, \mathrm{CDCl}_{3}$

$$
4 d
$$




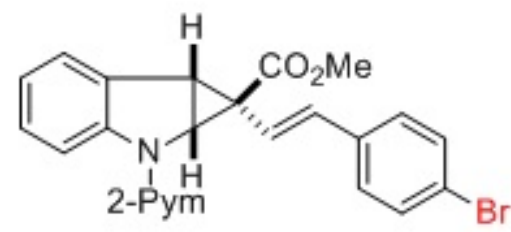

$400 \mathrm{MHz}, \mathrm{CDCl}_{3}$

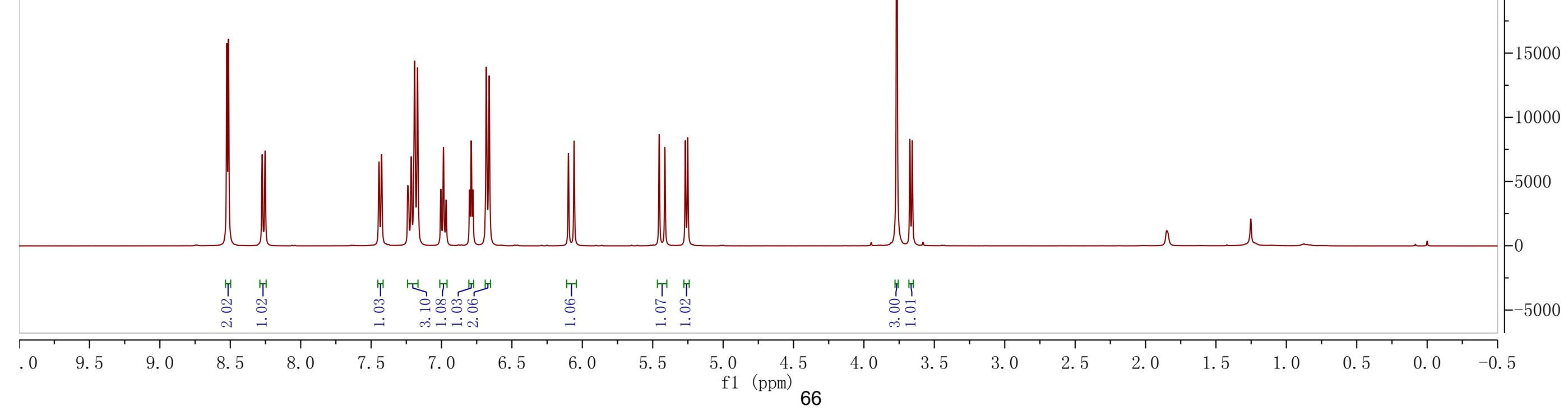




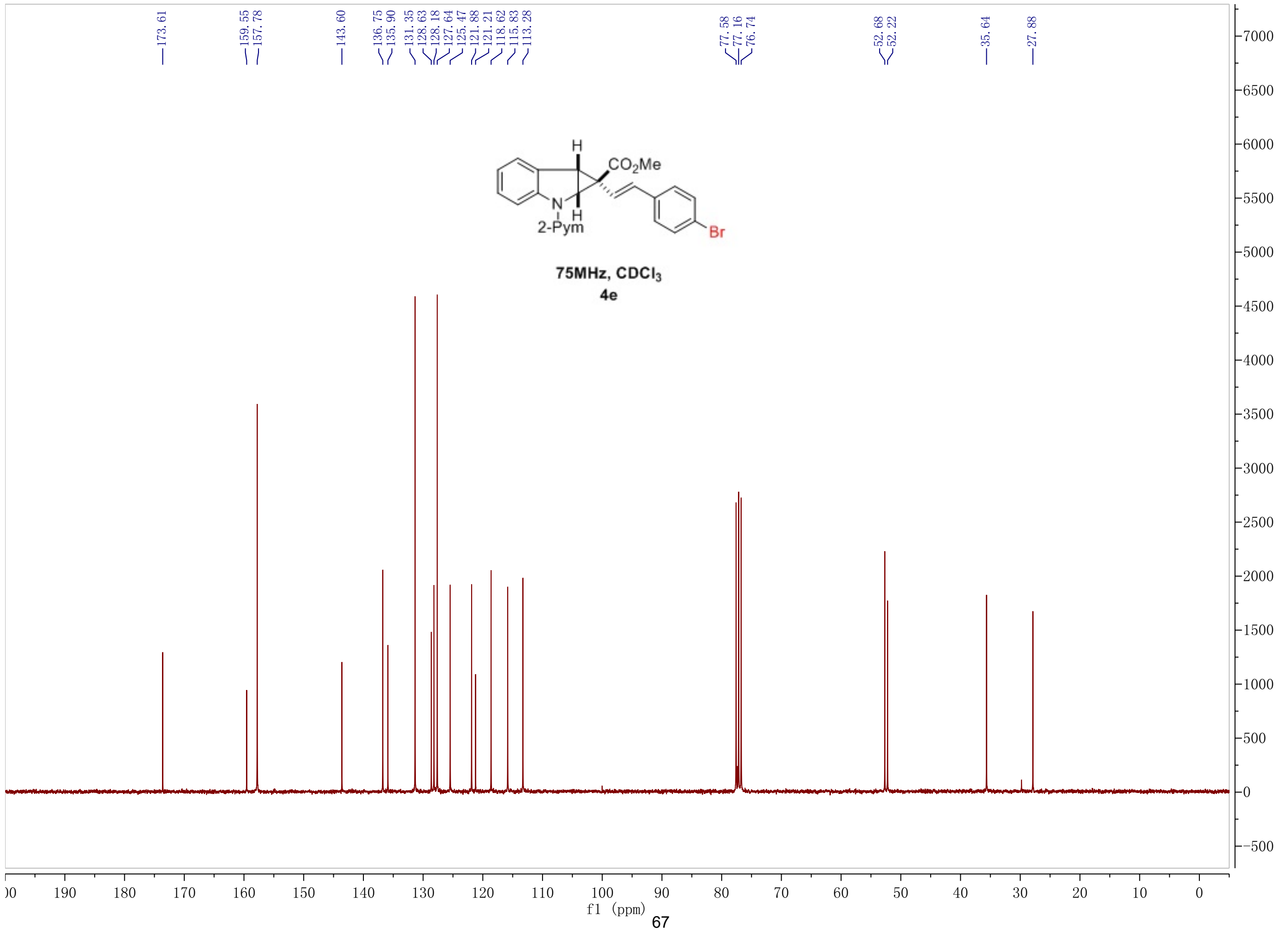




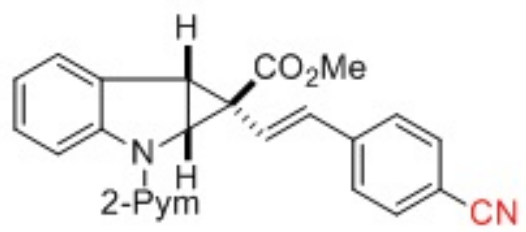

$300 \mathrm{MHz}, \mathrm{CDCl}_{3}$

$$
4 f
$$

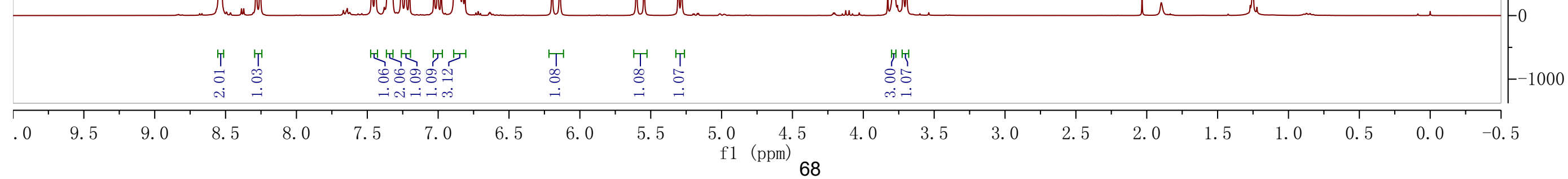




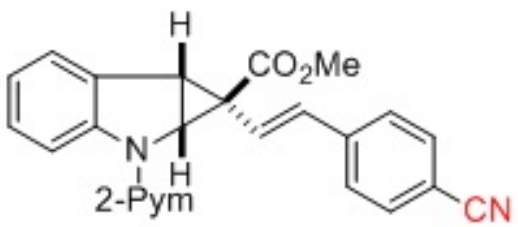

$100 \mathrm{MHz}, \mathrm{CDCl}_{3}$

$4 f$ 


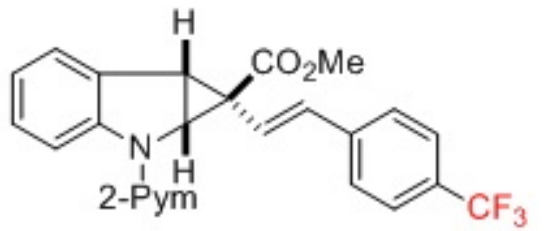

$400 \mathrm{MHz}, \mathrm{CDCl}_{3}$

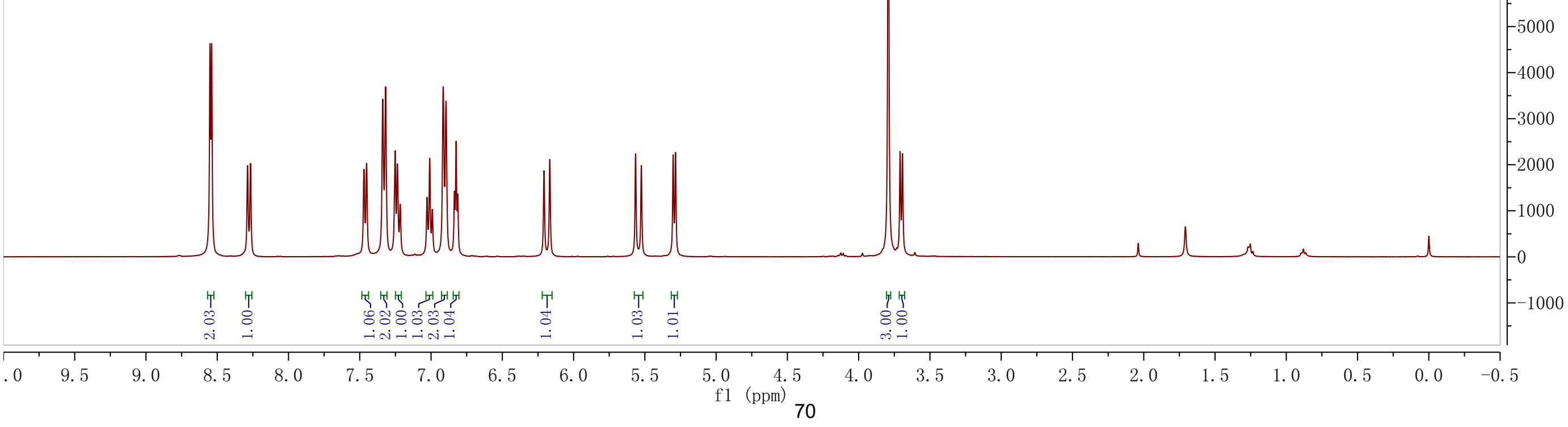




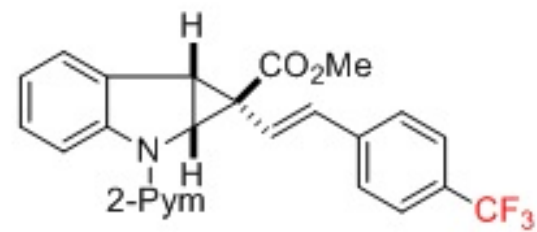

$75 \mathrm{MHz}, \mathrm{CDCl}_{3}$ 


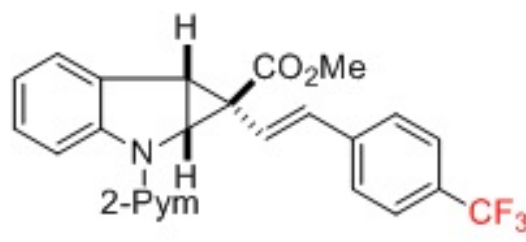

$282 \mathrm{MHz} \mathrm{CDCl}_{3}$

$4 \mathrm{~g}$ 


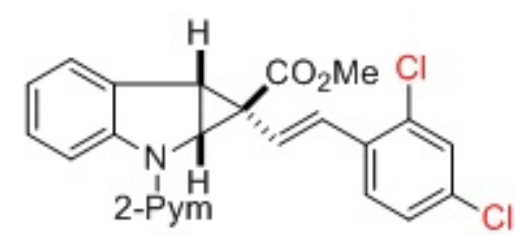

$400 \mathrm{MHz}, \mathrm{CDCl}_{3}$

$4 \mathrm{~h}$

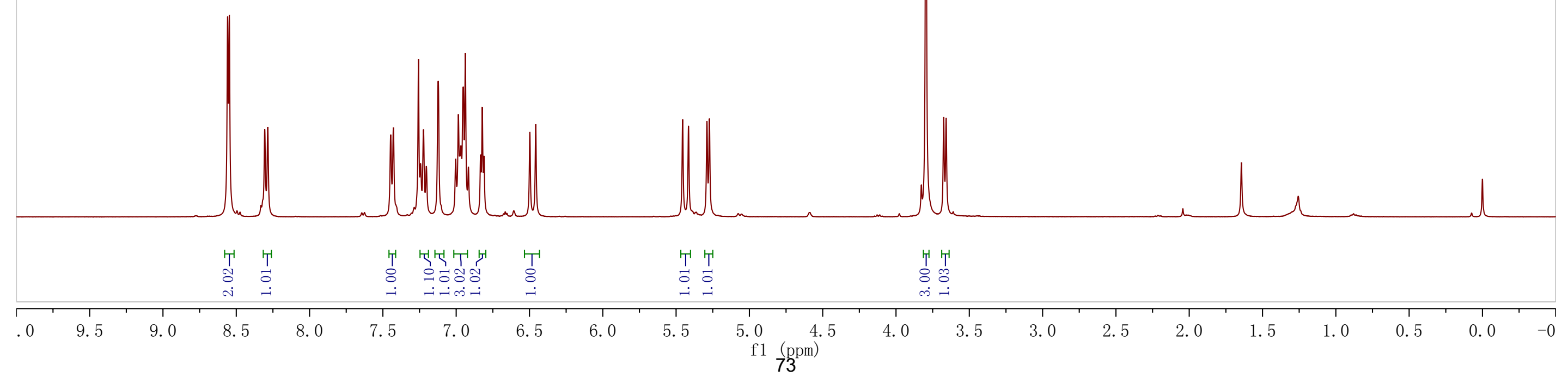




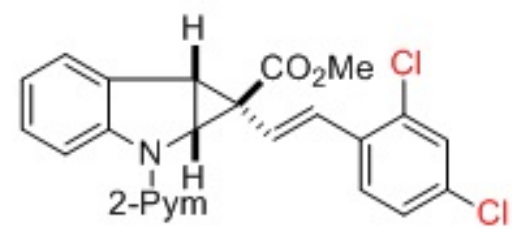

$75 \mathrm{MHz}, \mathrm{CDCl}_{3}$

$4 \mathrm{~h}$

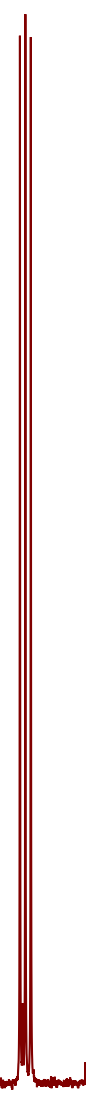




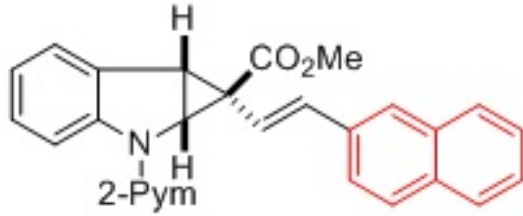

$300 \mathrm{MHz}, \mathrm{CDCl}_{3}$

$4 \mathrm{i}$ 


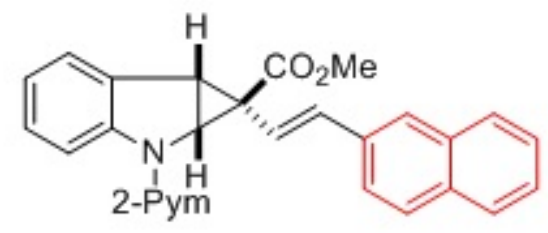

$75 \mathrm{MHz} \mathrm{CDCl}_{3}$

$4 i$ 


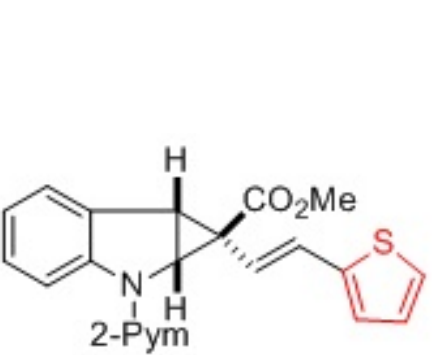

$300 \mathrm{MHz}, \mathrm{CDCl}_{3}$

4j

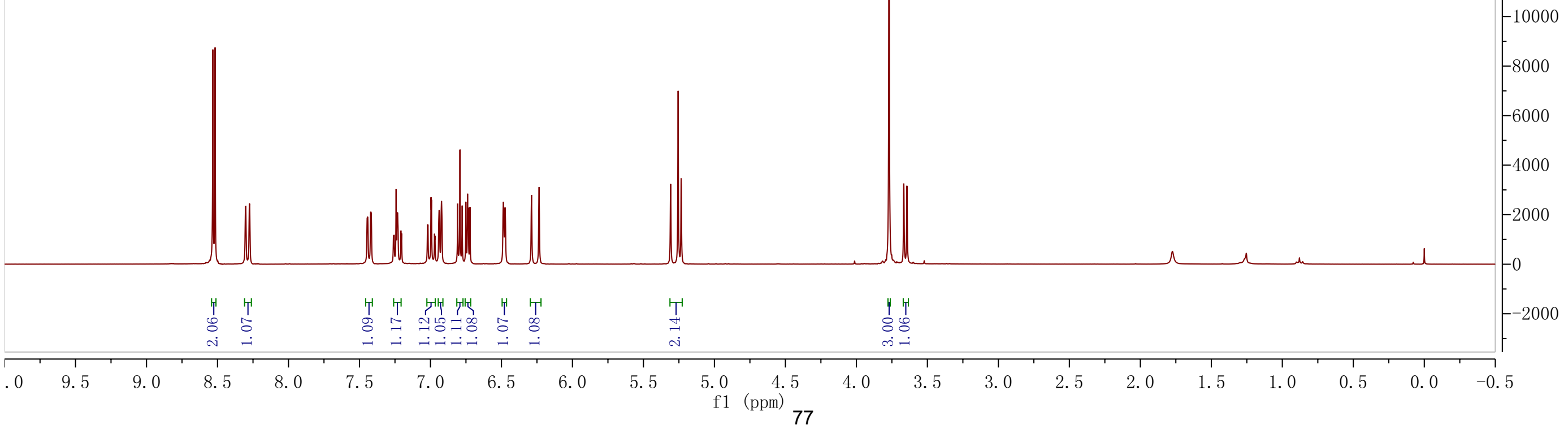




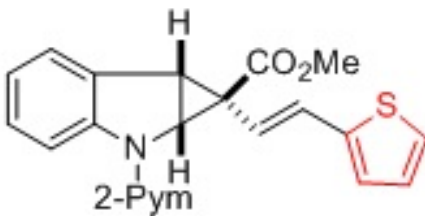

$75 \mathrm{MHz}, \mathrm{CDCl}_{3}$

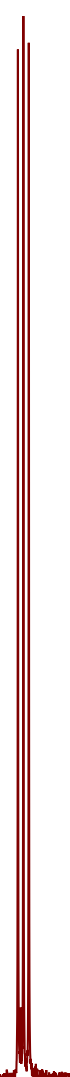




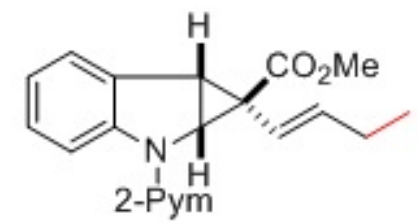

$400 \mathrm{MHz}, \mathrm{CDCl}_{3}$

$4 k$

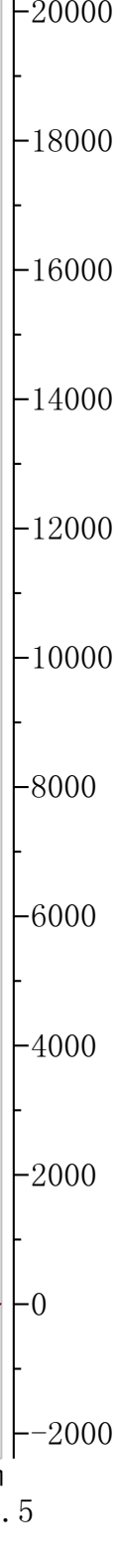




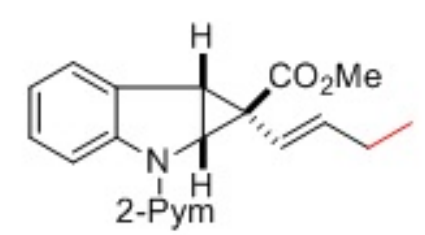

$75 \mathrm{MHz}, \mathrm{CDCl}_{3}$

$4 k$ 


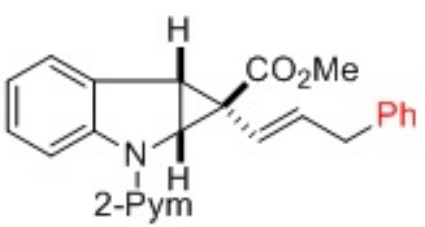

$400 \mathrm{MHz}, \mathrm{CDCl}_{3}$

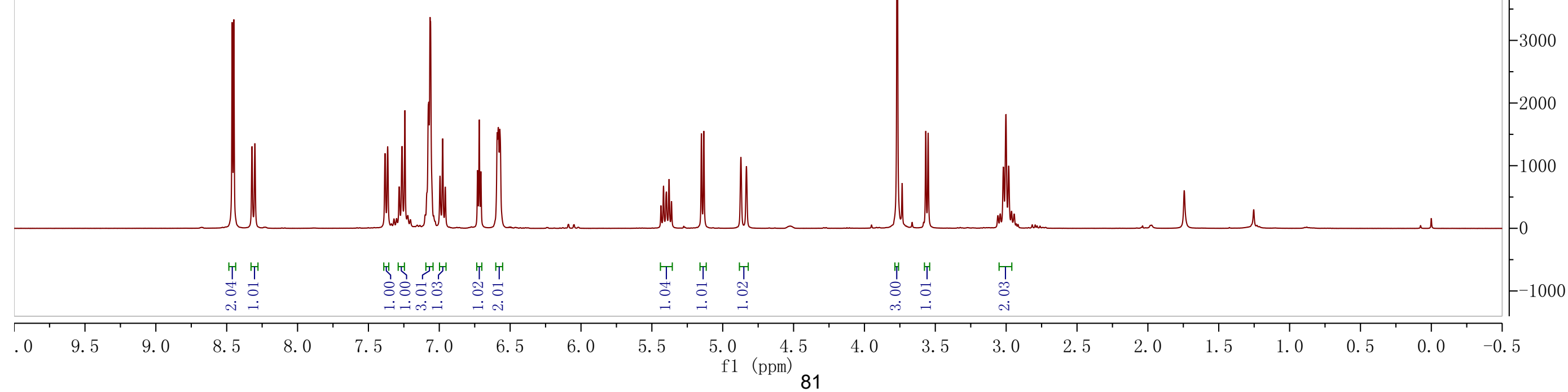




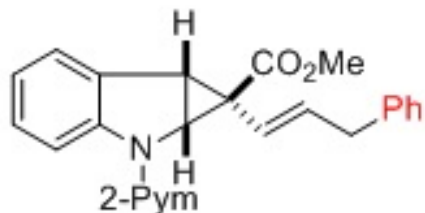

$75 \mathrm{MHz}, \mathrm{CDCl}_{3}$ 4 I

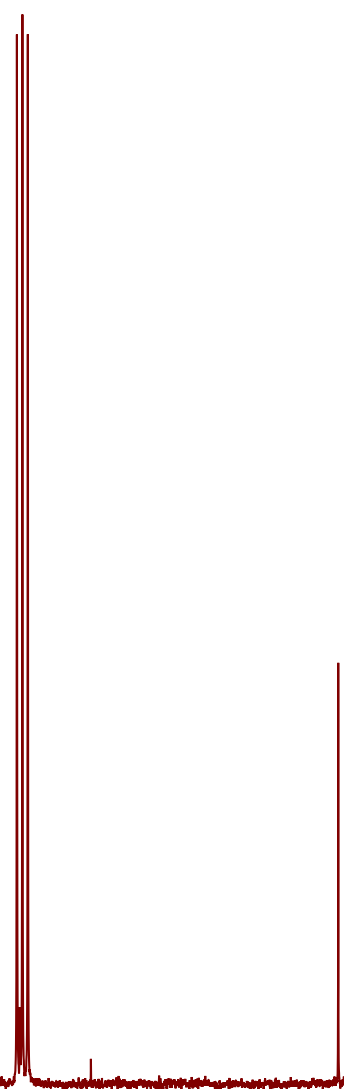




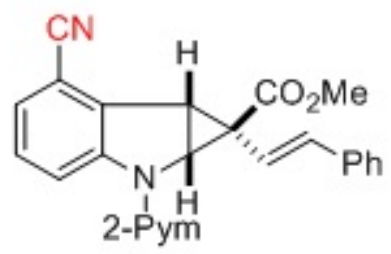

$400 \mathrm{MHz} \mathrm{CDCl}_{3}$

$4 \mathrm{~m}$

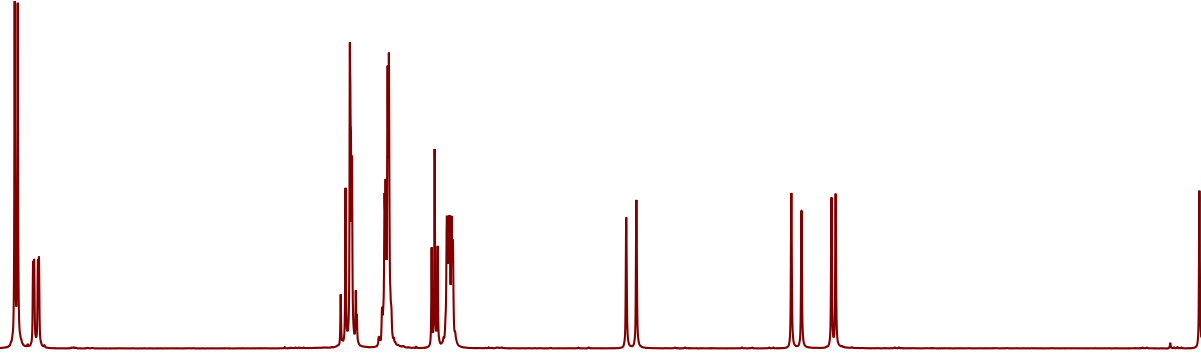

$\mid$

16000

15000

14000

13000 


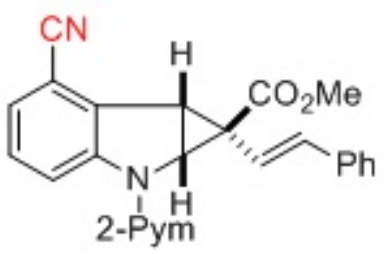

$75 \mathrm{MHz}, \mathrm{CDCl}_{3}$ 


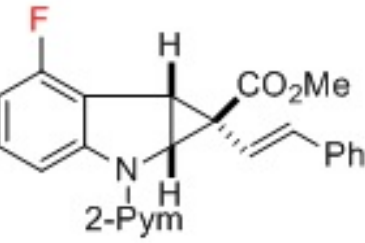

$300 \mathrm{MHz} \mathrm{CDCl}_{3}$

$4 n$

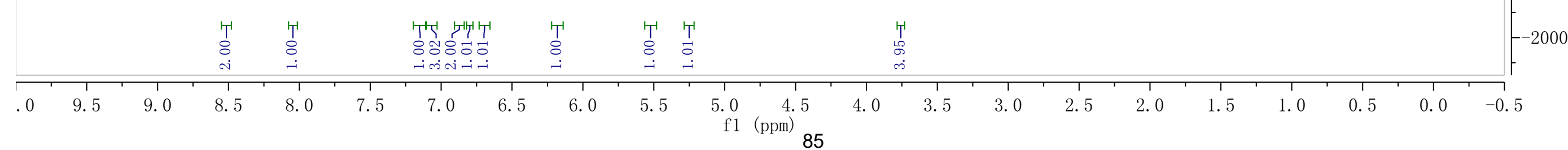




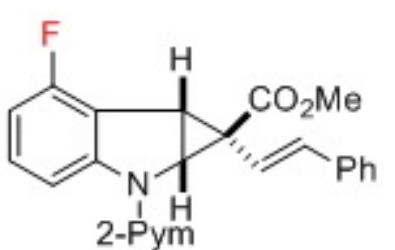

$282 \mathrm{MHz} \mathrm{CDCl}_{3}$

$4 n$ 


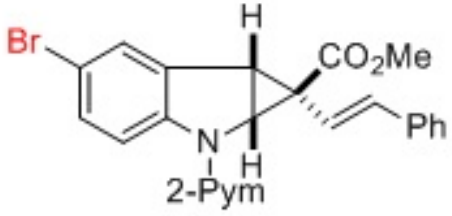

$400 \mathrm{MHz}, \mathrm{CDCl}_{3}$

40 


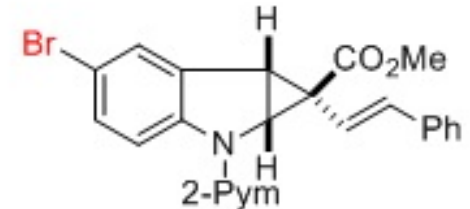

$100 \mathrm{MHz}, \mathrm{CDCl}_{3}$ 


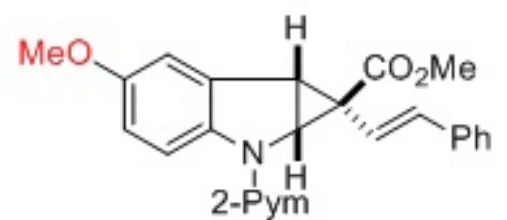

$400 \mathrm{MHz}, \mathrm{CDCl}_{3}$
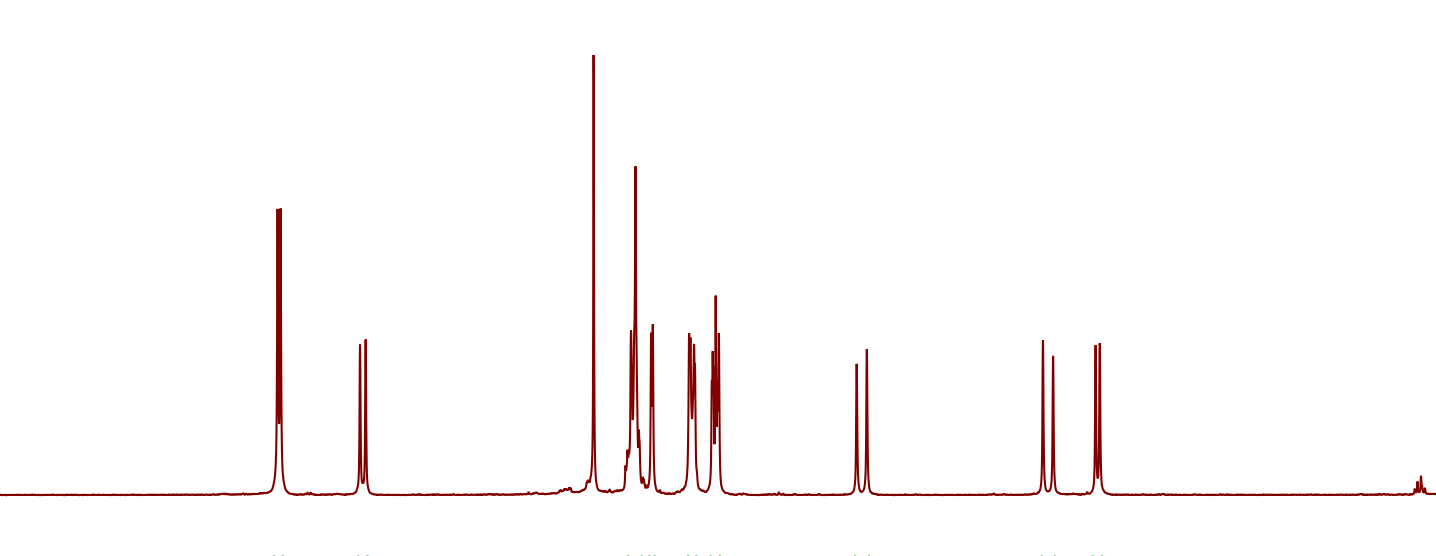

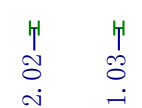

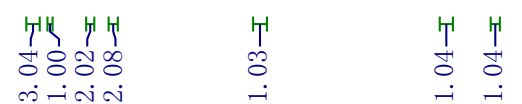

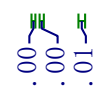




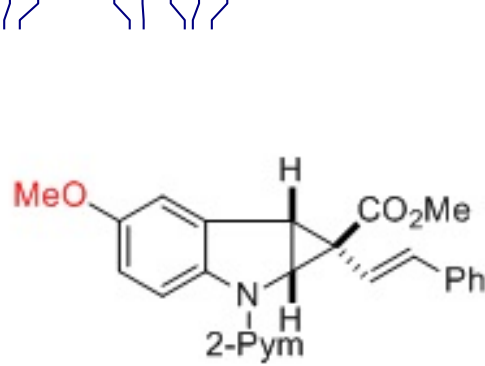

$75 \mathrm{MHz}, \mathrm{CDCl}_{3}$ 


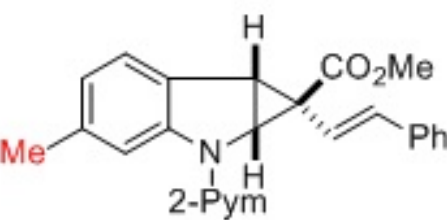

$400 \mathrm{MHz}, \mathrm{CDCl}_{3}$

$4 q$

\begin{tabular}{|c|c|c|c|c|c|c|c|c|c|c|c|c|c|c|c|c|c|c|c|c|c|}
\hline .0 & 9.5 & 9.0 & 8.5 & 8.0 & 7.5 & 7. 0 & 6.5 & 6.0 & 5.5 & $\begin{array}{r}5.0 \\
\text { f1 }\end{array}$ & $\begin{array}{r}4.5 \\
\text { ppm) }\end{array}$ & 4. 0 & 3. 5 & 3. 0 & 2.5 & 2. 0 & 1.5 & 1.0 & 0.5 & 0.0 & -0.5 \\
\hline
\end{tabular}




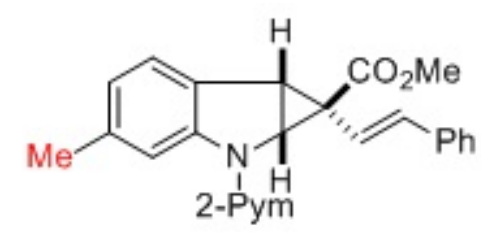

$75 \mathrm{MHz} \mathrm{CDCl}_{3}$

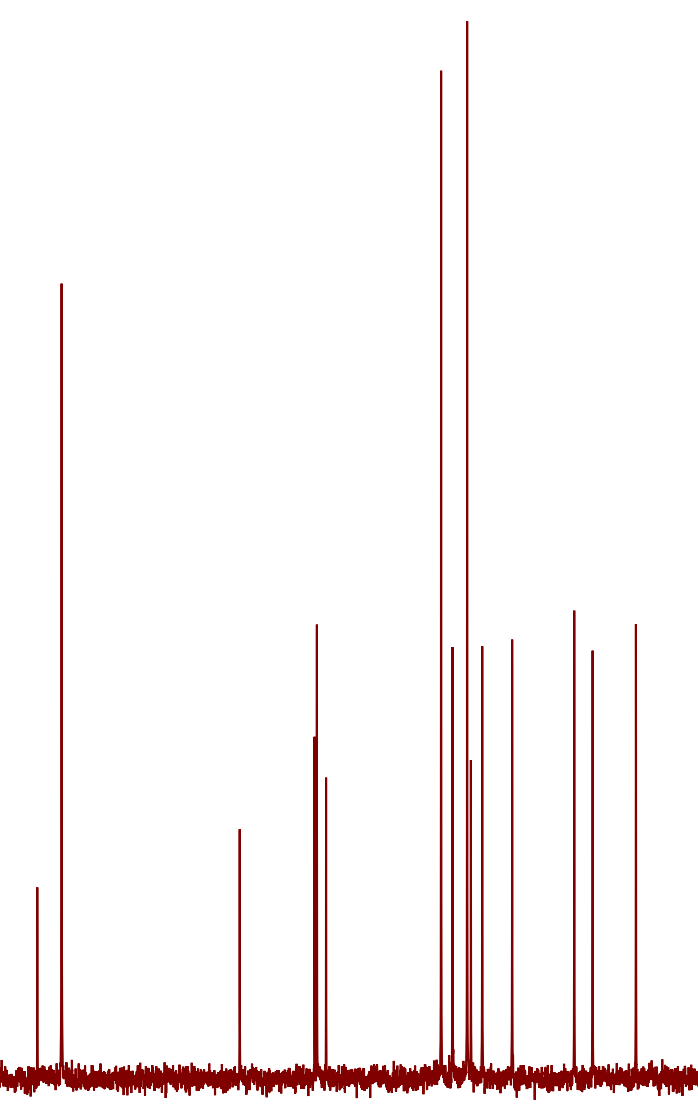




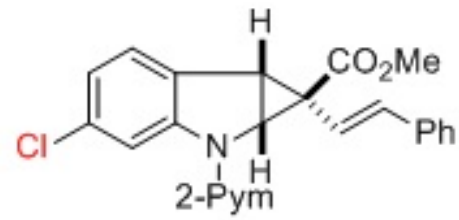

$400 \mathrm{MHz}, \mathrm{CDCl}_{3}$

$4 r$

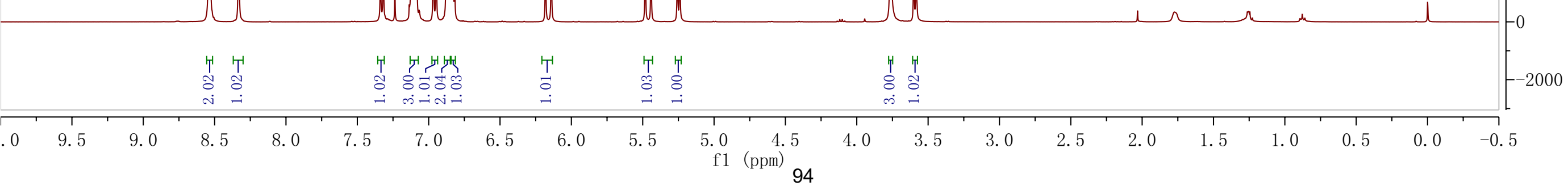




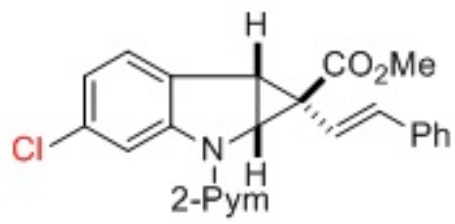

$100 \mathrm{MHz}, \mathrm{CDCl}_{3}$ 


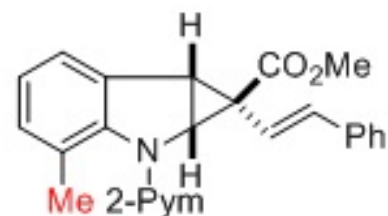

\section{$400 \mathrm{MHz}, \mathrm{CDCl}_{3}$}

$4 \mathrm{~s}$
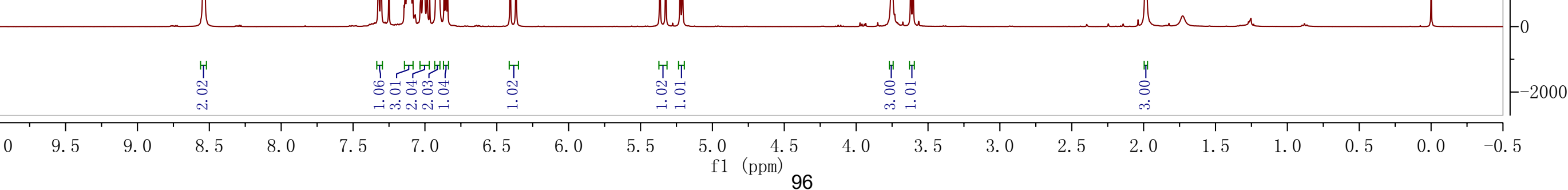


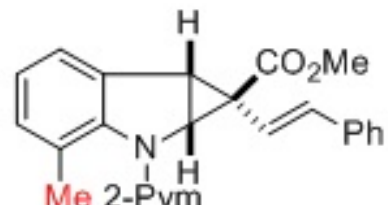

$100 \mathrm{MHz}, \mathrm{CDCl}_{3}$

$4 \mathrm{~s}$ 


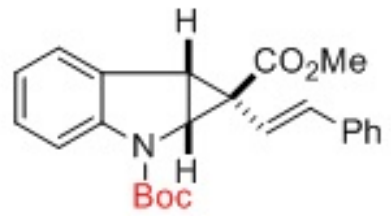

$300 \mathrm{MHz}, \mathrm{CDCl}_{3}$

$4 \mathrm{t}$

rotamer

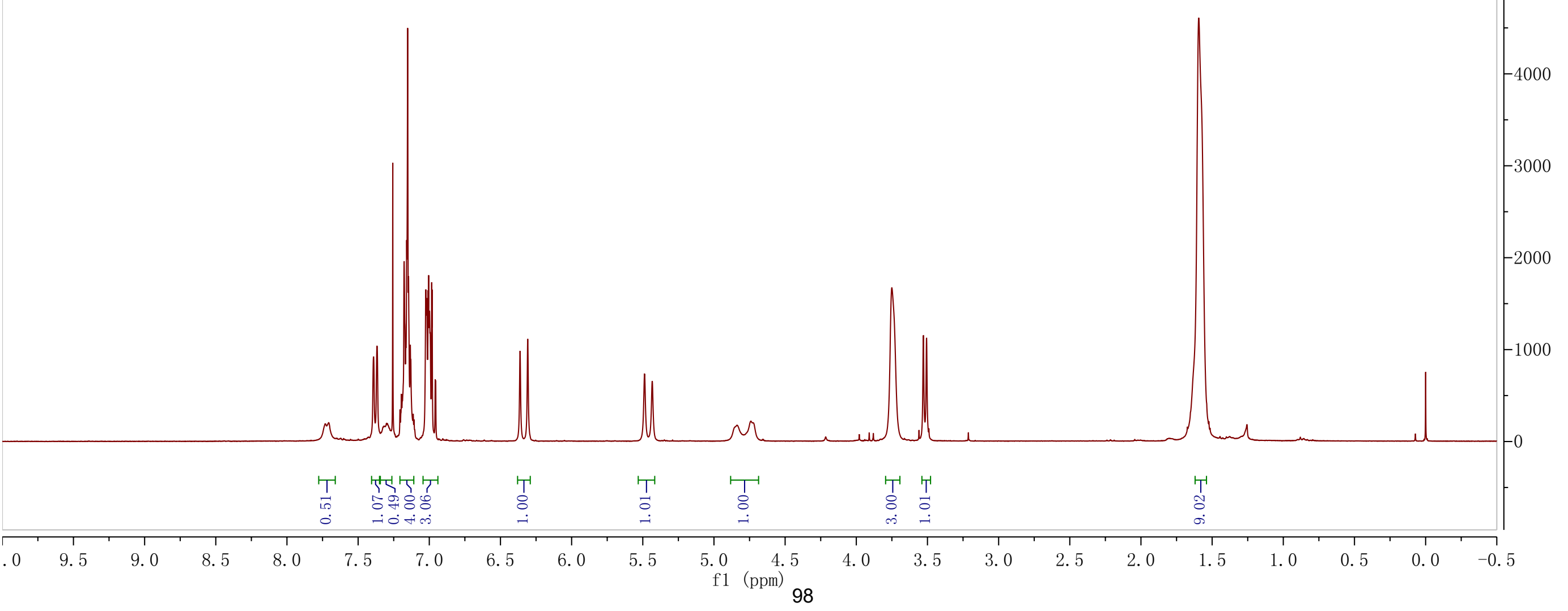




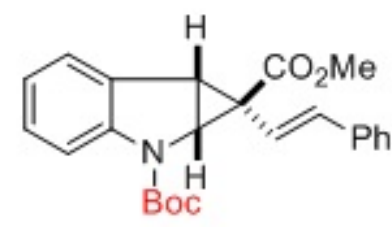

$75 \mathrm{MHz}, \mathrm{CDCl}_{3}$

$4 t$

\section{rotarmer}

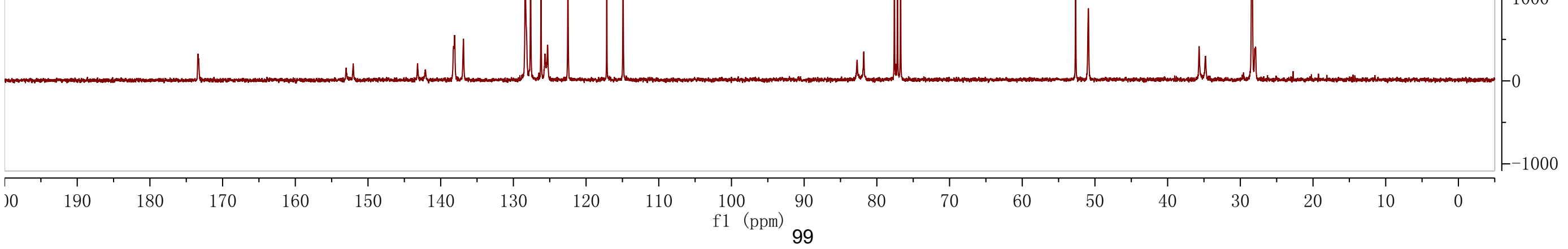


$4 \mathrm{u}$

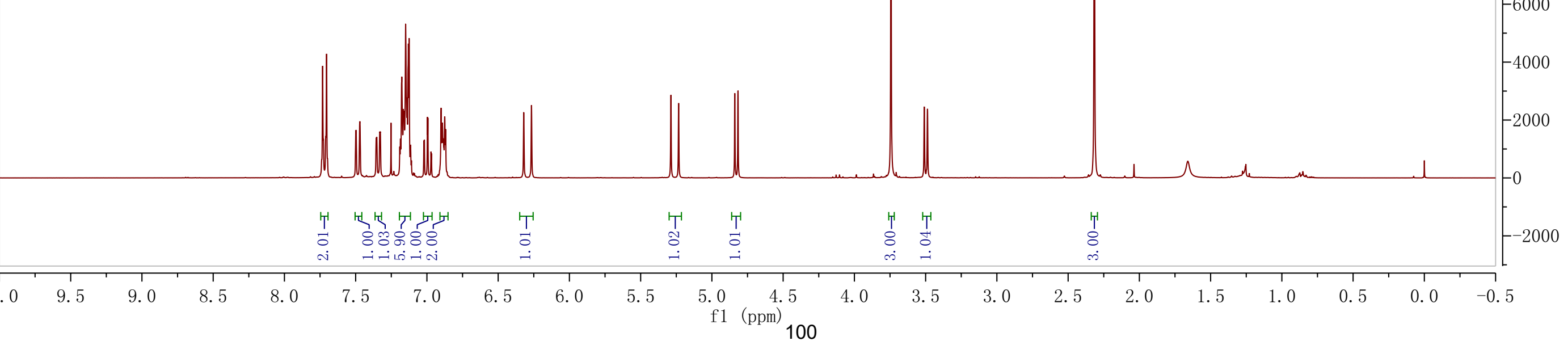




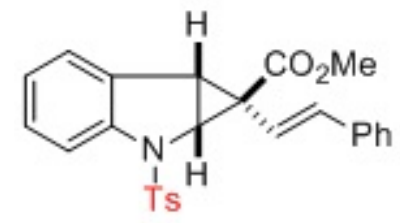

$4 \mathrm{u}$ 


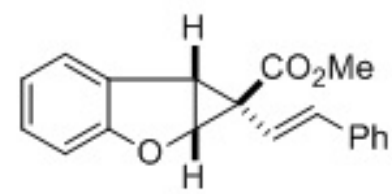

$400 \mathrm{MHz}, \mathrm{CDCl}_{3}$

6

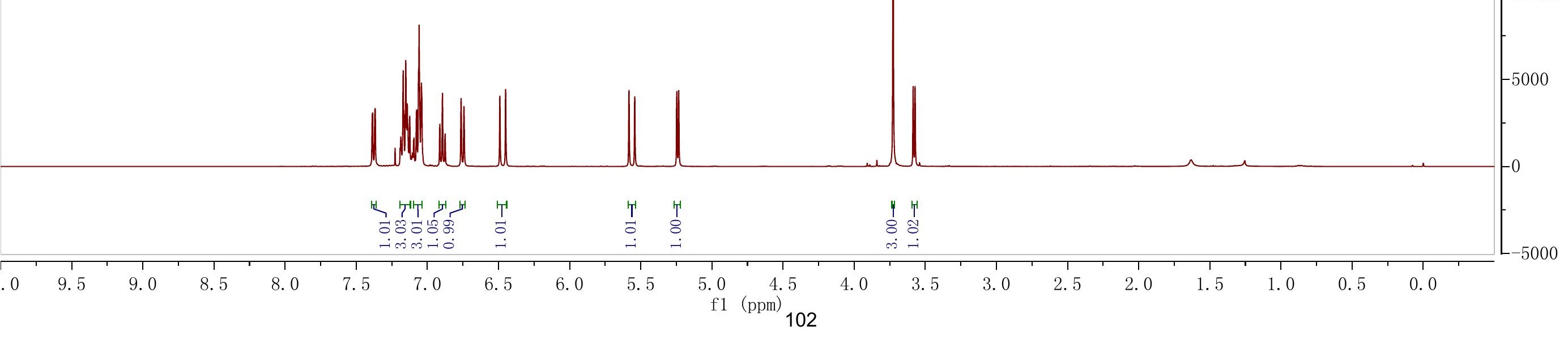




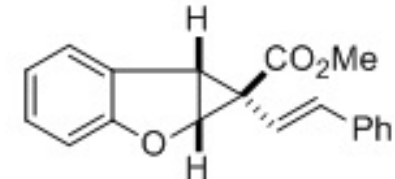

$100 \mathrm{MHz}, \mathrm{CDCl}_{3}$

$190 \quad 180 \quad 170 \quad 160$

$\begin{array}{rrrr}1 & & \\ 150 & 140 & 130\end{array}$

$130 \quad 120$

$20 \quad 110$

$\begin{array}{cccc}100 & 90 & 80 \\ \mathrm{f} 1(\mathrm{ppm}) & & \\ & & & \\ & & \end{array}$




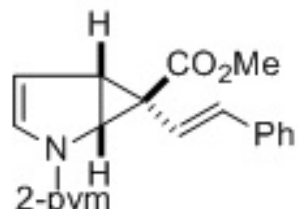

$400 \mathrm{MHz} \mathrm{CDCl}_{3}$

8

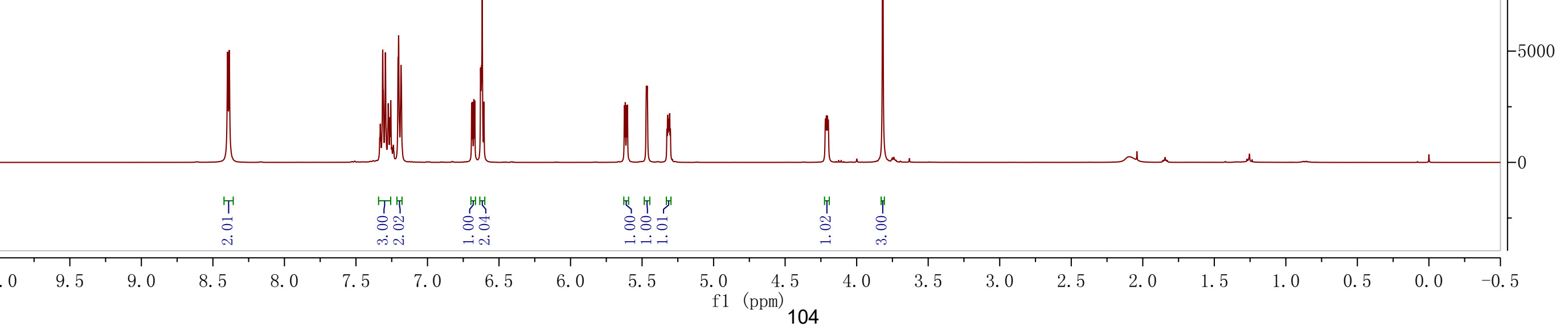




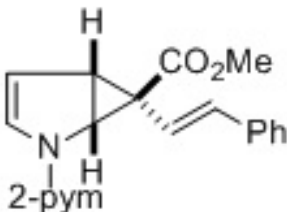

$100 \mathrm{MHz}, \mathrm{CDCl}_{3}$ 